PHYSICAL REVIEW D 94, 052012 (2016)

\title{
Search for two Higgs bosons in final states containing two photons and two bottom quarks in proton-proton collisions at $8 \mathrm{TeV}$
}

\author{
V. Khachatryan et al. \\ (CMS Collaboration) \\ (Received 22 March 2016; published 29 September 2016)
}

\begin{abstract}
A search is presented for the production of two Higgs bosons in final states containing two photons and two bottom quarks. Both resonant and nonresonant hypotheses are investigated. The analyzed data correspond to an integrated luminosity of $19.7 \mathrm{fb}^{-1}$ of proton-proton collisions at $\sqrt{s}=8 \mathrm{TeV}$ collected with the CMS detector. Good agreement is observed between data and predictions of the standard model (SM). Upper limits are set at $95 \%$ confidence level on the production cross section of new particles and compared to the prediction for the existence of a warped extra dimension. When the decay to two Higgs bosons is kinematically allowed, assuming a mass scale $\Lambda_{\mathrm{R}}=1 \mathrm{TeV}$ for the model, the data exclude a radion scalar at masses below $980 \mathrm{GeV}$. The first Kaluza-Klein excitation mode of the graviton in the RS1 Randall-Sundrum model is excluded for masses between 325 and $450 \mathrm{GeV}$. An upper limit of $0.71 \mathrm{pb}$ is set on the nonresonant two-Higgs-boson cross section in the SM-like hypothesis. Limits are also derived on nonresonant production assuming anomalous Higgs-boson couplings.
\end{abstract}

DOI: $10.1103 /$ PhysRevD.94.052012

\section{INTRODUCTION}

The discovery of a boson with a mass of approximately $125 \mathrm{GeV}$, with properties close to those expected for the Higgs boson $(H)$ of the standard model (SM) [1,2], has stimulated interest in the exploration of the Higgs potential. The production of a pair of Higgs bosons $(\mathrm{HH})$ is a rare process that is sensitive to the structure of this potential through the self-coupling mechanism of the Higgs boson. In the SM, the cross section for the production of two Higgs bosons in proton-proton $(p p)$ collisions at $8 \mathrm{TeV}$ is $10.0 \pm$ $1.4 \mathrm{fb}$ for the gluon-gluon fusion process [3-5], which lies beyond the reach of analyses based on the first run of the CERN LHC.

Many theories beyond the SM (BSM) suggest the existence of heavy particles that can couple to a pair of Higgs bosons. These particles could appear as a resonant contribution in the invariant mass of the $\mathrm{HH}$ system. If the new particles are too heavy to be observed through a direct search, they may be sensed in the HH production through their virtual contributions (as shown, e.g., in Refs. [6,7]); also, the fundamental couplings of the model can be modified relative to their SM values (as shown, e.g., in Refs. $[8,9])$; in both cases, a nonresonant enhancement of the $\mathrm{HH}$ production could be observed.

Models with a warped extra dimension (WED), as proposed by Randall and Sundrum [10], postulate the existence of one spatial extra dimension compactified

*Full author list given at the end of the article.

Published by the American Physical Society under the terms of the Creative Commons Attribution 3.0 License. Further distribution of this work must maintain attribution to the author(s) and the published article's title, journal citation, and DOI. between two fixed points, commonly called branes. The region between the branes is referred to as bulk, and controlled through an exponential metric. The gap between the two fundamental scales of nature, such as the Planck scale $\left(M_{\mathrm{Pl}}\right)$, and the electroweak scale, is controlled by a warp factor $(k)$ in the metric, which corresponds to one of the fundamental parameters of the model. The brane where the density of the extra dimensional metric is localized is called "Planck brane," while the other, where the Higgs field is localized, is called "TeV brane." This class of models predicts the existence of new particles that can decay to a Higgs-boson pair, such as the spin-0 radion ( $\mathrm{R})$ [11-13], and the spin-2 first Kaluza-Klein (KK) excitation of the graviton [14-16].

There are two possible ways of describing a KK graviton in WED that depend on the choice of localization for the SM matter fields. In the RS1 model, only gravity is allowed to propagate in the extradimensional bulk. In this model the couplings of the KK graviton to matter fields are controlled by $k / \bar{M}_{\mathrm{Pl}}[10]$, with the reduced Planck mass $\bar{M}_{\mathrm{Pl}}$ defined by $M_{\mathrm{Pl}} / \sqrt{8 \pi}$. For the possibility of $\mathrm{SM}$ particles to propagate in the bulk (the so-called bulk-RS model), the coupling of the KK graviton to matter depends on the choice for the localization of the SM bulk fields. This paper uses the phenomenology of Ref. [17], where SM particles are allowed to propagate in the bulk, and follows the characteristics of the SM gauge group, with the righthanded top quark localized on the $\mathrm{TeV}$ brane (so-called elementary top hypothesis).

The $\mathrm{R}$ is an additional element of WED models that is needed to stabilize the size of the extra dimension $l$. It is usual to express the benchmark points of the model in terms of the dimensionless quantity $k / \bar{M}_{\mathrm{Pl}}$, and the mass scale $\Lambda_{\mathrm{R}}=\sqrt{6} \exp [-k l] \bar{M}_{\mathrm{Pl}}$, with the latter interpreted as the 
ultraviolet cutoff of the model [18]. The addition of a scalar-curvature term can induce a mixing between the scalar radion and the Higgs boson $[18,19]$. This possibility is discussed, for example, in Ref. [20]. Precision electroweak studies suggest that this mixing is expected to be small [21]. In our interpretations of the constraints we neglect the possibility of Higgs-radion mixing.

On one hand, the choice of localization of the SM matter fields for the KK-graviton resonance impacts the kinematics of the signal and drastically modifies the production and decay properties [22]. The physics of the radion, on the other hand, does not depend much on the choice of the model [18], which obviates the need to distinguish the RS1 and bulk-RS possibilities.

Models with an extended Higgs sector also predict one spin-0 resonance that, when sufficiently massive, decays to a pair of SM Higgs bosons, and corresponds to an additional Higgs boson. Examples of such models are the singlet extension [23], the two Higgs doublet models [24] (in particular, the minimal supersymmetric model $[25,26]$ ), and the Georgi-Machacek model [27]. The majority of these models predict that heavy scalar production occurs predominantly through the gluon-gluon fusion process. The Lorentz structure of the coupling between the scalar and the gluon is the same for a radion or a heavy Higgs boson. Therefore the models for the production of a radion or an additional Higgs boson are essentially the same, provided the interpretations are performed in a parameter space region where the spin- 0 resonance is narrow. The results of this paper can therefore be easily applied to constrain this class of models.

Phenomenological explorations of the two-Higgs-boson channel were studied prior to the observation of the Higgs boson [28], and, since then, other studies have become available [29-35]. Most of these indicate that in BSM physics an enhancement of the $H H$ production cross section is expected, together with modified signal kinematics for the $H H$ final state. This paper describes a search for the production of pairs of Higgs bosons in the $\gamma \gamma b \bar{b}$ final state in pp collisions at the LHC, using data corresponding to an integrated luminosity of $19.7 \mathrm{fb}^{-1}$ collected by the CMS experiment at $\sqrt{s}=8 \mathrm{TeV}$. Both nonresonant and resonant production are explored, with the search for a narrow resonance $\mathrm{X}$ conducted at masses $m_{\mathrm{X}}$ between 260 and $1100 \mathrm{GeV}$.

The fully reconstructed $\gamma \gamma b \bar{b}$ final state discussed in this paper combines the large SM branching fraction $(\mathcal{B})$ of the $H \rightarrow b \bar{b}$ decay with the comparatively low background and good mass resolution of the $H \rightarrow \gamma \gamma$ channel, yielding a total $\mathcal{B}(H H \rightarrow \gamma \gamma b \bar{b})$ of $0.26 \%$ [36]. The search exploits the mass spectra of the diphoton $\left(m_{\gamma \gamma}\right)$, dijet $\left(m_{\mathrm{jj}}\right)$, and the four-body systems $\left(m_{\gamma \gamma \mathrm{jj}}\right)$, as well as the direction of Higgs bosons in the Collins-Soper frame [37], to provide discrimination between production of two Higgs bosons and SM background.
A search in the same final state was performed by the ATLAS collaboration [38]. Complementary final states such as $H H \rightarrow b \bar{b} b \bar{b}, H H \rightarrow \tau \tau b \bar{b}$, and $H H$ to multileptons and multiphotons were also explored by the ATLAS [39,40] and CMS [41-44] collaborations.

This paper is organized as follows: Section II contains a brief description of the CMS detector. In Sec. III we describe the simulated signal and background event samples used in the analysis. Section IV is dedicated to the discussion of event selection and Higgs-boson reconstruction. The signal extraction procedure is discussed in Sec. V. In Sec. VI we present the systematic uncertainties impacting each analysis method. Section VII contains the results of resonant and nonresonant searches, and Sec. VIII provides a summary.

\section{THE CMS DETECTOR}

The CMS detector, its coordinate system, and main kinematic variables used in the analysis are described in detail in Ref. [45]. The detector is a multipurpose apparatus designed to study physics processes at large transverse momentum $p_{\mathrm{T}}$ in $\mathrm{pp}$ and heavy-ion collisions. The central feature of the apparatus is a superconducting solenoid, of $6 \mathrm{~m}$ internal diameter, providing a magnetic field of $3.8 \mathrm{~T}$. A silicon pixel and strip tracker covering the pseudorapidity range $|\eta|<2.5$, a crystal electromagnetic calorimeter (ECAL), and a brass and scintillator hadron calorimeter (HCAL) reside within the field volume. The ECAL is made of lead tungstate crystals, while the HCAL has layers of plates of brass and plastic scintillator. These calorimeters are both composed of a barrel and two endcap sections and provide coverage up to $|\eta|<3.0$. An iron and quartz-fiber Cherenkov hadron calorimeter covers larger values of $3.0<|\eta|<5.0$. Muons are measured in the $|\eta|<2.4$ range, using detection planes based on three technologies: drift tubes, cathode strip chambers, and resistive-plate chambers.

The first level of the CMS trigger system, composed of special hardware processors, uses information from the calorimeters and muon detectors to select the most interesting events in a time interval of less than $4 \mu \mathrm{s}$. The highlevel trigger (HLT) processor farm further decreases the event rate from around $100 \mathrm{kHz}$ to less than $1 \mathrm{kHz}$, before data storage.

\section{SIMULATED EVENTS}

The MAdGraPH version 5.1.4.5 [46] Monte Carlo (MC) program generates parton-level signal events based on matrix element calculations at leading order (LO) in quantum chromodynamics (QCD), using LO PYTHIA version 6.426 [47] for showering and hadronization of partons. The models provide a description of production through gluon-gluon fusion of particles with narrow width (width set to $1 \mathrm{MeV}$ ) that decay to two Higgs bosons, with mass $m_{H}=125 \mathrm{GeV}$, in agreement with Ref. [48]. 
Events are generated either for spin-0 radion production, or spin-2 KK-graviton production predicted by the bulkRS model.

The samples for nonresonant production are generated considering the cross section dependence on three parameters: the Higgs-boson trilinear coupling $\lambda$, parametrized as $\kappa_{\lambda} \equiv \lambda / \lambda^{\mathrm{SM}}$, where $\lambda^{\mathrm{SM}} \equiv m_{H}^{2} /\left(2 v^{2}\right)=0.129$, with $v=$ $246 \mathrm{GeV}$ being the vacuum expectation value of the Higgs boson; the top Yukawa coupling $y_{t}$, parametrized as $\kappa_{t} \equiv y_{t} / y_{t}{ }^{\mathrm{SM}}$, where $y_{t}^{\mathrm{SM}}=m_{t} / v$ is the SM value of the top Yukawa coupling, and $m_{t}$ the top quark mass; and the coefficient $c_{2}$ of a possible coupling of two Higgs bosons to two top quarks. The first two parameters reflect changes relative to SM values, while the third corresponds purely to a BSM operator. In this parametrization the SM production corresponds to the point $\kappa_{\lambda}=1, \kappa_{t}=1$, and $c_{2}=0$. The parameters $\kappa_{\lambda}$ and $c_{2}$ cannot be directly constrained by alternative measurements at the LHC. Therefore we vary these parameters in a wide range: $-20 \leq \kappa_{\lambda} \leq 20$ and $-3 \leq c_{2} \leq 3$. The range $0.75 \leq \kappa_{t} \leq 1.25$ is compatible with constraints from the single Higgs-boson measurements provided in Ref. [49].

The part of the Higgs potential $\Delta \mathcal{L}$ relevant to twoHiggs-boson production and their interactions with the top quark can be expressed as in Ref. [50],

$\Delta \mathcal{L}=\kappa_{\lambda} \lambda^{\mathrm{SM}} v H^{3}-\frac{m_{t}}{v}\left(v+\kappa_{t} H+\frac{c_{2}}{v} H H\right)\left(\bar{t}_{\mathrm{L}} t_{\mathrm{R}}+\right.$ H.c. $)$,

where $t_{\mathrm{L}}$ and $t_{\mathrm{R}}$ are the top quark fields with left and right chiralities, respectively, and $H$ is the physical Higgsboson field.

Besides being used to predict SM single-Higgs-boson production, the MC predictions for the background processes are used also in comparisons with data, to optimize the selection criteria, and for checking backgroundestimation methods based on control samples in data. The dominant background, originating from events with two prompt photons and two jets in the final state, is generated at next-to-leading order (NLO) in QCD using SHERPA version 1.4.2 [51]. Multijet production with or without a single-prompt photon represents a subdominant background, and is generated with the PYTHIA 6 package. Other minor backgrounds, including Drell-Yan $\left(p p \rightarrow Z / \gamma^{*} \rightarrow e^{+} e^{-}\right.$), SM Higgs-boson production with jets, as well as vector boson and top quark production in association with photons, are generated using MADGRAPH and PYTHIA 6, or the generator POWHEG version 1.0 [52-54] at NLO in QCD. The generated events are processed through GEANT4-based [55,56] detector simulation.

\section{EVENT RECONSTRUCTION}

The events are selected using two complementary HLT paths requiring two photons. The first trigger requires an identification based on the energy distribution of the electromagnetic shower and loose isolation requirements on photon candidates. The second trigger applies tighter constraints on the shower shape, but a looser kinematic selection. The trigger thresholds on the $p_{\mathrm{T}}$ range between 26 and $36 \mathrm{GeV}$, and between 18 and $22 \mathrm{GeV}$, respectively, for photons with highest (leading) and next-to-highest (subleading) $p_{\mathrm{T}}$, with specific choices that depend on the instantaneous LHC luminosity. The HLT paths are more than $99 \%$ efficient for the selection criteria used in this analysis [57].

\section{A. The $\boldsymbol{H} \rightarrow \gamma \gamma$ candidate}

Photon candidates are constructed from clusters of energy in the ECAL $[58,59]$. They are subsequently calibrated [60] and identified through a cutoff-based approach (referred to as "cut-based analysis" in Ref. [57]). The identification criteria include requirements on $p_{\mathrm{T}}$ of the electromagnetic shower, its longitudinal leakage into the HCAL, its isolation from jet activity in the event, as well as a veto on the presence of a track matching the ECAL cluster. These criteria provide efficient rejection of objects that arise from jets or electrons but are reconstructed as photons. Both photons are required to be within the ECAL fiducial volume of $\left|\eta_{\gamma}\right|<2.5$. Small transition regions between the ECAL barrel and the ECAL endcaps are excluded in this analysis, because the reconstruction of a photon object in this region is not optimal.

The directions of the photons are reconstructed assuming that they arise from the primary vertex of the hard interaction. However on average $\approx 20$ additional pp interactions (pileup) occur in the same or neighboring pp bunch crossings as the main interaction. Many additional vertexes are therefore usually reconstructed in an event using charged particle tracks. We assume that the primary interaction vertex corresponds to the one that maximizes the sum in $p_{\mathrm{T}}^{2}$ of the associated charged particle tracks. For the simulated signal, it is shown that this choice of vertex lies within $1 \mathrm{~cm}$ of the true hard-interaction vertex in $99 \%$ of the events. With this choice for energy reconstruction and vertex identification, the diphoton mass resolution remains close to $1 \mathrm{GeV}$ independent of the signal hypothesis.

Diphoton candidates are preselected by requiring $100<m_{\gamma \gamma}<180 \mathrm{GeV}$. The two photons are further required to satisfy the asymmetric selection criteria $p_{\mathrm{T}}^{\gamma 1} / m_{\gamma \gamma}>1 / 3$ and $p_{\mathrm{T}}^{\gamma 2} / m_{\gamma \gamma}>1 / 4$, where $p_{\mathrm{T}}^{\gamma 1}$ and $p_{\mathrm{T}}^{\gamma 2}$ are the transverse momenta of the leading and subleading photons. The use of different $p_{\mathrm{T}}$ thresholds scaled by the diphoton invariant mass minimizes turn-on effects that can distort the distribution at the low-mass end of the $m_{\gamma \gamma}$ spectrum. If there is more than one diphoton candidate selected through the above requirements, the pair with the largest scalar sum in the $p_{\mathrm{T}}$ of the two photons is chosen for analysis. 


\section{B. The $H \rightarrow b \bar{b}$ candidate}

The Higgs-boson candidate decaying into two $b$ quarks is reconstructed following a procedure similar to that used in CMS searches for SM Higgs bosons that decay to $b$ quarks [61].

The particle-flow event algorithm reconstructs and identifies each individual particle (referred to as candidates) with an optimized combination of information from the various elements of the CMS detector $[62,63]$. Then the anti- $k_{\mathrm{T}}$ algorithm [64] clusters particle-flow candidates into jets using a distance parameter $D=0.5$. Jets are required to be within the tracker acceptance $\left(\left|\eta_{\mathrm{j}}\right|<2.4\right)$, and separated from both photons through a condition on the angular distance in $\eta \times \phi$ space of $\Delta R_{\gamma \mathrm{j}} \equiv \sqrt{(\Delta \eta)^{2}+(\Delta \phi)^{2}}>0.5$, where $\phi$ is the azimuth angle in radians. The jet energy is corrected for extra depositions from pileup interactions, using the jet-area technique [65] implemented in the FASTJET package [66]. Jet energy corrections are applied as a function of $\eta_{\mathrm{j}}$ and $p_{\mathrm{T}}^{\mathrm{j}}[67,68]$. Identification criteria are applied to reject detector noise misidentified as jets, and the procedure is verified using simulated signal.

The identification of jets likely to have originated from hadronization of $b$ quarks exploits the combined secondary vertex (CSV) $b$ quark tagger [69]. This algorithm combines the information from track impact parameters and secondary vertexes within a given jet into a continuous output discriminant. Jets with CSV tagger values above some fixed threshold are considered as $b$ tagged. The working point chosen in this analysis corresponds to an efficiency, estimated from simulated multijet events, of $\approx 70 \%$ and a mistag rate for light quarks and gluons of $1 \%-2 \%$, depending on jet $p_{\mathrm{T}}$. This efficiency and the mistag rate are measured in data samples enriched in $b$ jets (e.g., in $t \bar{t}$ events). Correction factors of $\approx 0.95$ are determined from data-to-simulation comparisons and applied as weights to all simulated events.

Events are kept if at least two jets are selected and at least one of them is $b$ tagged. To improve signal sensitivity, events are subsequently classified in two categories: events with exactly one $b$-tagged jet (medium purity) and events with more than one $b$-tagged jet (high purity). In the former category, the $H \rightarrow b \bar{b}$ decay is reconstructed by pairing the $b$-tagged jet with a non- $b$-tagged jet, while in the latter category a pair of $b$-tagged jets is used. In both cases, when multiple pairing possibilities exist for the Higgs-boson candidate, the dijet system with largest $p_{\mathrm{T}}$ is retained for further study. For medium- and high-purity simulated signal events, this procedure selects the correct jets in more than $80 \%$ and more than $95 \%$, respectively.

The resolution in $m_{\mathrm{jj}}$ improves from $20 \mathrm{GeV}$ for $m_{\mathrm{X}}=$ $300 \mathrm{GeV}$ to $15 \mathrm{GeV}$ for $m_{\mathrm{X}}=1 \mathrm{TeV}$ in the high-purity category, and from $25 \mathrm{GeV}$ for $m_{\mathrm{X}}=300 \mathrm{GeV}$ to $15 \mathrm{GeV}$ for $m_{\mathrm{X}}=1 \mathrm{TeV}$ in the medium-purity category. In the search for a low-mass resonance, the dijet mass resolution is improved using a multivariate regression technique [61] that uses the global information from the events as well as the particular properties of each jet, in an attempt to identify the semileptonic decays of $B$ mesons and correct for the energy carried away by undetected neutrinos. The relative improvement in resolution is typically $15 \%$. For the highmass analysis and nonresonant analysis the $m_{\mathrm{jj}}$ resolution is better than for low-mass analysis. The improvement provided by the regression technique was found to be very limited. Therefore in those cases no regression was used.

Independent of whether a search involves the usage of jet energy regression, all jets are required to have $p_{\mathrm{T}}^{\mathrm{j}}>25 \mathrm{GeV}$. Finally, we require that $60<m_{\mathrm{jj}}<$ $180 \mathrm{GeV}$.

\section{The two-Higgs-boson system}

The object selections discussed thus far are summarized in Table I.

In each category, two Higgs bosons are obtained by combining the diphoton and the dijet boson candidates. To improve the resolution in $m_{\gamma \gamma \mathrm{jj}}$, an additional constraint is imposed requiring $m_{\mathrm{jj}}$ to be consistent with $m_{H}$. This is achieved by modifying the jet 4-momenta using multiplicative factors. The value of each factor is obtained event by event through a $\chi^{2}$ minimization procedure where the size of the denominator is defined by the estimated resolution for each jet [70]. The procedure, similar to the one used in Ref. [70], is referred to as a kinematic fit and the resulting four-body mass is termed $m_{\gamma \gamma \mathrm{jij}}^{\mathrm{kin}}$ :

The scattering angle, $\theta_{H H}^{\mathrm{CS}}$, is defined in the CollinsSoper frame of the four-body system state, as the angle between the momentum of the Higgs boson decaying into two photons and the line that bisects the acute angle between the colliding protons. In the Collins-Soper frame, the two-Higgs-boson candidates are collinear, and the choice of the one decaying to photons as reference is therefore arbitrary. Using the absolute value of the cosine of this angle, $\left|\cos \theta_{H H}^{\mathrm{CS}}\right|$, obviates this arbitrariness.

\section{Backgrounds}

The SM background in $m_{\gamma \gamma}$ can be classified into two categories: the nonresonant background, from multijet and electroweak processes, and a peaking background corresponding to events from single Higgs bosons decaying to two photons.

TABLE I. Summary of the analysis preselections.

\begin{tabular}{lcccc}
\hline \hline \multicolumn{2}{c}{ Photons } & & \multicolumn{2}{c}{ Jets } \\
\cline { 1 - 3 } \cline { 5 - 6 } Variable & Range & & Variable & Range \\
\hline$p_{\mathrm{T}}^{\gamma 1} / m_{\gamma \gamma}$ & $>1 / 3$ & & $p_{\mathrm{T}}^{\mathrm{j}}(\mathrm{GeV})$ & $>25$ \\
$p_{\mathrm{T}}^{\gamma 2} / m_{\gamma \gamma}$ & $>1 / 4$ & & $\left|\eta_{\mathrm{j}}\right|$ & $<2.4$ \\
$\left|\eta_{\gamma}\right|$ & $<2.5$ & & $m_{\mathrm{jj}}(\mathrm{GeV})$ & {$[60,180]$} \\
$m_{\gamma \gamma}(\mathrm{GeV})$ & {$[100,180]$} & & $b$-tagged jets & $>0$ \\
\hline \hline
\end{tabular}



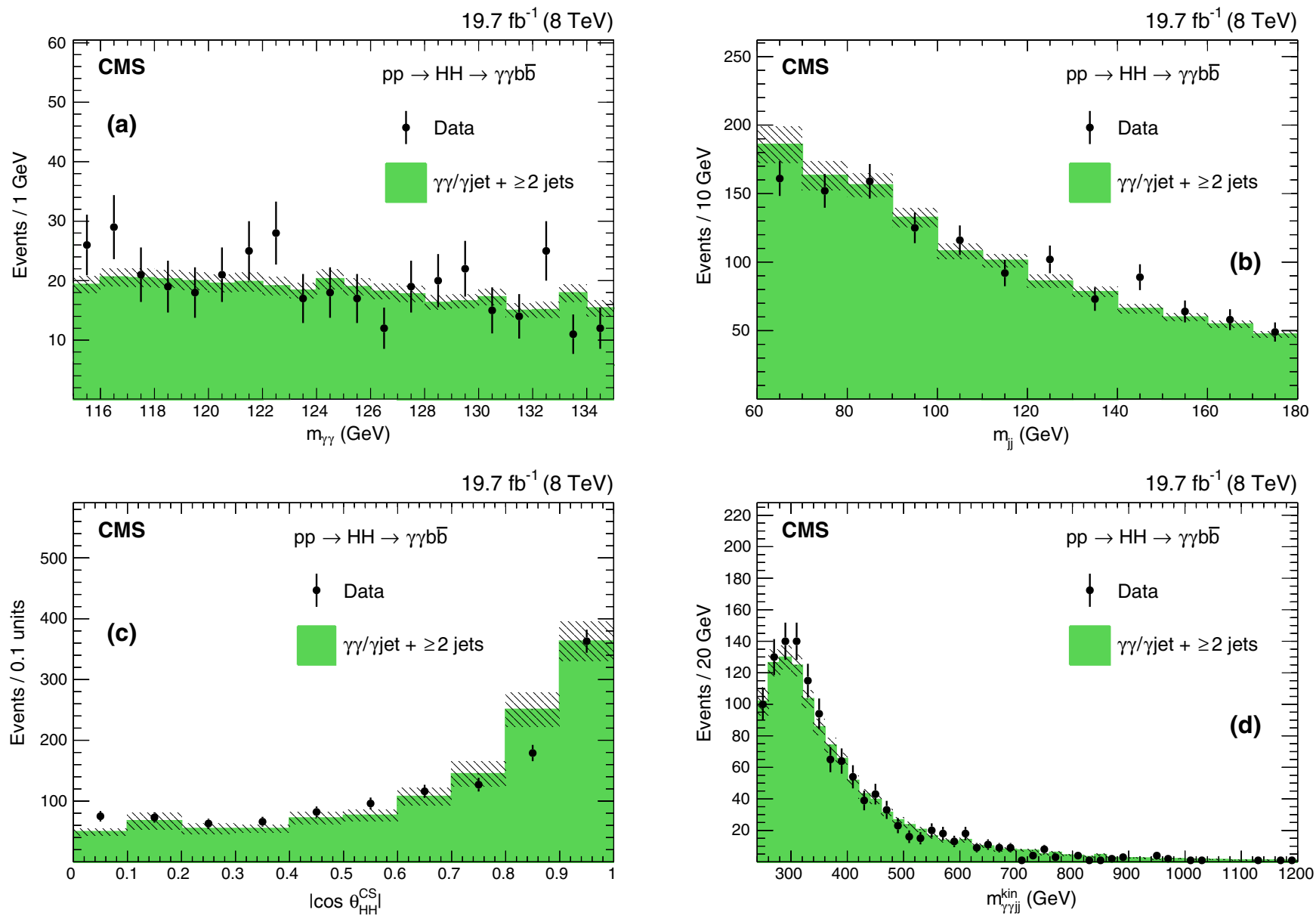

FIG. 1. Reconstructed spectra for data compared to the $\gamma \gamma / \gamma$ jet $+\geq 2$ jets background after the selections described in Table I (selections on photons and jets and a requirement of at least one $b$-tagged jet): (a) $m_{\gamma \gamma}$, (b) $m_{\mathrm{jj}}$, (c) $\left|\cos \theta_{H H}^{\mathrm{CS}}\right|$, and (d) $m_{\gamma \gamma \mathrm{jj}}^{\mathrm{kin}}$ The hatched area corresponds to the bin-by-bin statistical uncertainties on the background prediction reflecting the limited size of the generated MC sample. The comparison is provided for illustrative purpose, the backgrounds, except the one coming from single-Higgs-boson production, are evaluated from a fit to the data without reference to the MC simulation.

After the baseline selections of Table I, the dominant nonresonant background with two prompt photons and more than two extra jets, referred to as $\gamma \gamma+\geq 2$ jets, represents $\approx 75 \%$ of the total background. The nonresonant background with one prompt photon and a jet misidentified as a photon as well as more than two extra jets, referred to as $\gamma$ jet $+\geq 2$ jets, represents in turn $\approx 25 \%$. The background from two jets misidentified as photons is negligible.

The remaining nonresonant and resonant backgrounds contribute much less than $1 \%$ to the total. They represent associated production of photons with top quarks or single electroweak bosons decaying to quarks, and Drell-Yan events with their decay electrons misidentified as photons. The resonant backgrounds correspond to different SM processes contributing to single-Higgs-boson production.

All nonresonant backgrounds are estimated from data, and the resonant background from SM single-Higgs-boson production in different channels is taken from the MC simulation normalized to NLO or next-to-NLO (NNLO) production cross sections, whichever are available [36].
The comparison between data and $\mathrm{MC}$ predictions is provided in Fig. 1. The $\gamma \gamma / \gamma$ jet $+\geq 2$ jets background is normalized to the total integral of data in the signal free region, defined by the condition $m_{\gamma \gamma}>130$ or $m_{\gamma \gamma}<$ $120 \mathrm{GeV}$ in addition to the selections of Table I.

\section{ANALYSIS METHODS}

In the final step, this analysis exploits kinematic properties of the final state to discriminate either the resonant or nonresonant signal from SM background: the Higgs boson masses $m_{\gamma \gamma}$ and $m_{\mathrm{j} j}$, the cosine of their scattering angle $\left|\cos \theta_{H H}^{\mathrm{CS}}\right|$, and the mass of the two-Higgs-boson system, $m_{\gamma \gamma \mathrm{jj}}^{\mathrm{kin}}$. Distributions in these variables are shown for different signal assumptions in Fig. 2. The signal peaks in $m_{\gamma \gamma}$ and $m_{\mathrm{jj}}$ are shown in Figs. 2(a) and 2(b). The corresponding distributions for the QCD background are smoothly varying over the shown ranges. The $\left|\cos \theta_{H H}^{\mathrm{CS}}\right|$ is rather uniform for signal, as shown in Fig. 2(c), while it peaks toward 1 for background. Finally, a resonant signal appears as a narrow 

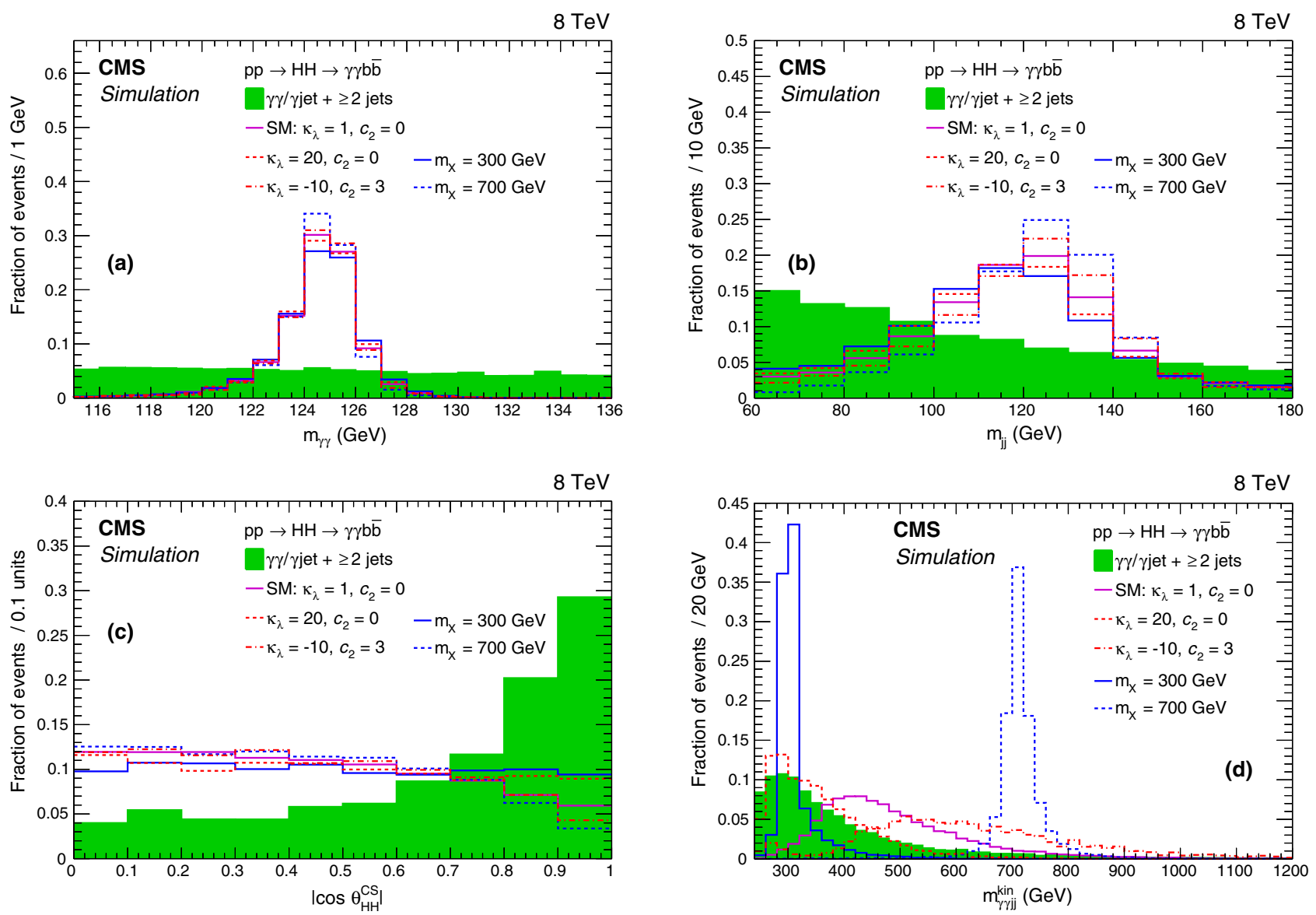

FIG. 2. Simulated spectra for the spin- 0 radion signal at $m_{\mathrm{X}}=300$ and $700 \mathrm{GeV}$, and for some values of the anomalous couplings, compared to SM Higgs-boson production and QCD background, after the selections described in Table I (selections on photons and jets and a requirement of at least one $b$-tagged jet): (a) $m_{\gamma \gamma}$, (b) $m_{\mathrm{jj}}$, (c) $\left|\cos \theta_{H H}^{\mathrm{CS}}\right|$, and (d) $m_{\gamma \gamma \mathrm{jj}}^{\mathrm{kin}}$. All spectra are normalized to unity.

peak in the $m_{\gamma \gamma \mathrm{jj}}^{\mathrm{kin}}$ spectrum, while the nonresonant signal has a broad contribution as shown in Fig. 2(d).

The dominant background from nonresonant production of prompt photons and jets exhibits a kinematic peak around $m_{\gamma \gamma \mathrm{jj}}^{\mathrm{kin}} \approx 300 \mathrm{GeV}$ followed by a slowly falling tail at high $m_{\gamma \gamma \mathrm{jj}}^{\mathrm{kin}}$. In the resonant case, we consider two strategies, one for $m_{\mathrm{X}}$ close to the kinematic peak, and one for $m_{\mathrm{X}}$ heavier than the kinematic peak. A third strategy is considered for the nonresonant case, since the signal distribution as a function of $m_{\gamma \gamma \mathrm{jj}}^{\mathrm{kin}}$ is broad. In all cases a categorization is used based on the number of $b$-tagged jets. All the strategies are summarized in Table II and briefly described below.

(1) Resonant search in the low-mass region (260 $\leq$ $m_{\mathrm{X}} \leq 400 \mathrm{GeV}$ ): the events are selected in a narrow window around the $m_{\mathrm{X}}$ hypothesis in the $m_{\gamma \gamma \mathrm{ji}}^{\mathrm{kin}}$ spectrum, and the signal is identified simultaneously in the $m_{\gamma \gamma}$ and $m_{\mathrm{jj}}$ spectra. This approach avoids a direct search for a resonance in the $m_{\gamma \gamma \mathrm{jij}}^{\mathrm{kin}}$ spectrum near the top of the kinematic peak of the SM background.

(2) Resonant search in the high-mass region (400 $\leq$ $\left.m_{\mathrm{X}} \leq 1100 \mathrm{GeV}\right)$ : the events are selected in a window around $m_{H}$ in both the $m_{\gamma \gamma}$ and $m_{\mathrm{jj}}$ spectra, and the signal is identified in the $m_{\gamma \gamma \mathrm{jj}}^{\mathrm{kin}}$ spectrum.
(3) Nonresonant search: a selection is applied in the $\left|\cos \theta_{H H}^{\mathrm{CS}}\right|$ variable to reduce the background. In addition to the categorization in the number of $b$-tagged jets, a categorization is applied in $m_{\gamma \gamma \mathrm{jj}}^{\mathrm{kin}}$ by defining a high-mass region and a low-mass region. The signal is identified simultaneously in the $m_{\gamma \gamma}$ and $m_{\mathrm{jj}}$ spectra.

The nonresonant background is described through different functions such as exponentials, power law, or polynomials in the Bernstein basis [57]. When the search is performed simultaneously in the diphoton and dijet mass spectra, these functions are used to construct a two-dimensional (2D) probability density (PD) for the background in each category, following an approach similar to Ref. [71]. Otherwise, a one-dimensional (1D) PD is used. In all cases,

TABLE II. Summary of the search analysis methods.

\begin{tabular}{lccc}
\hline \hline Signal hypothesis & Select & No. of categories & Fit \\
\hline (1) $m_{\mathrm{X}} \leq 400 \mathrm{GeV}$ & $m_{\gamma \gamma \mathrm{jj}}^{\mathrm{kin}}$ & $2(b$ tags $)$ & $m_{\gamma \gamma}, m_{\mathrm{jj}}$ \\
(2) $m_{\mathrm{X}} \geq 400 \mathrm{GeV}$ & $m_{\gamma \gamma}, m_{\mathrm{jj}}$ & $2(b$ tags $)$ & $m_{\gamma \gamma \mathrm{jj}}^{\mathrm{kin}}$ \\
(3) Nonresonant & $\left|\cos \theta_{H H}^{\mathrm{CS}}\right|$ & $4\left(b\right.$ tags, $\left.m_{\gamma \gamma \mathrm{ji}}^{\mathrm{kin}}\right)$ & $m_{\gamma \gamma}, m_{\mathrm{jj}}$ \\
\hline \hline
\end{tabular}



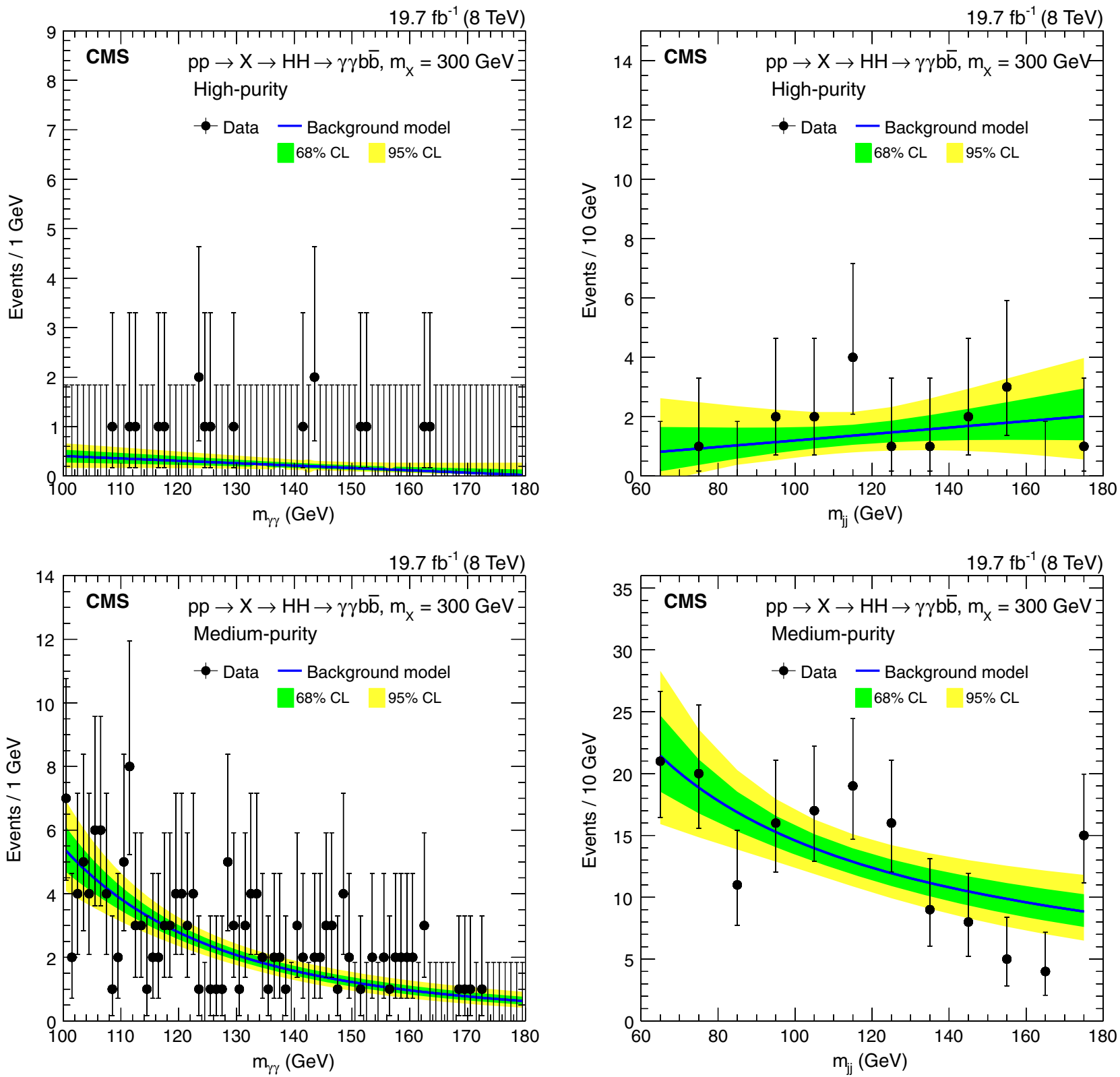

FIG. 3. Low-mass resonant analysis: fits to the nonresonant background contribution in the high-purity category to the $m_{\gamma \gamma}$ (top left) and $m_{\mathrm{jj}}$ spectra (top right), and similarly for the medium-purity category in the bottom left and bottom right, respectively. The fits to the background-only hypothesis are given by the blue curves, along with their $68 \%$ and $95 \%$ C.L. contours. The selections are designed to search for a $m_{\mathrm{X}}=300 \mathrm{GeV}$ hypothesis: $290<m_{\gamma \gamma \mathrm{jj}}^{\mathrm{kin}}<310 \mathrm{GeV}$.

we choose the background PD to minimize the bias on signal. The bias is always found to be at least a factor of 7 smaller than the statistical uncertainty in the fit, and can be safely neglected [1].

In each invariant mass distribution used to identify the signal, the signal PD is modeled, following the same approach as in Ref. [57], through the sum of a Gaussian function and a crystal ball (CB) function [72], using the parameters extracted from fits to $\mathrm{MC}$ simulations. The resolution parameters in both functions are kept independent, $\sigma_{\mathrm{x}}^{\mathrm{G}}$ for the Gaussian and $\sigma_{\mathrm{x}}^{\mathrm{CB}}$ for the $\mathrm{CB}$ function, but in the fits to each of the channels $(\mathrm{x}=\gamma \gamma, \mathrm{jj}, \gamma \gamma \mathrm{jj})$, we let the $\mu$ parameter for both the Gaussian and the CB component float, which provides three independent $\mu_{\mathrm{x}}$ values.

Finally, we consider the contribution from SM singleHiggs-boson production in 2D searches. The gluon-gluon and vector-boson fusion processes are modeled in $m_{\gamma \gamma}$ by a sum over Gaussian and CB functions, and through a constant term in $m_{\mathrm{jj}}$. The associated production of vector bosons that subsequently decay to jets, and the SM single Higgs bosons are modeled in the same way as the signal. The parameters of the distribution are extracted from a fit to the MC simulation. 
TABLE III. Additional selection criteria applied in the highmass resonant search.

\begin{tabular}{lcc}
\hline \hline & \multicolumn{2}{c}{ Range $(\mathrm{GeV})$} \\
\cline { 2 - 4 } Variable & Medium purity & High purity \\
\hline$m_{\gamma \gamma}$ & $122-128$ & $120-130$ \\
$m_{\mathrm{jj}}$ & & $85-170$ \\
\hline \hline
\end{tabular}

The total PD used for signal extraction corresponds to a sum over separate PD contributions from the signal component, single-Higgs boson production, and nonresonant backgrounds. We also verify that 2D PD functions can be considered as uncorrelated between $m_{\gamma \gamma}$ and $m_{\mathrm{jj}}$ within the statistical uncertainties. To obtain this result we calculated the correlation in data. The uncertainty in the correlation was estimated by generating pseudoexperiments from a model assuming no correlation between $m_{\gamma \gamma}$ and $m_{\mathrm{jj}}$ and calculating the root mean square of the resulting distribution.

\section{A. Low-mass resonant}

In addition to the preselections summarized in Table I, each mass hypothesis has a selection applied on $m_{\gamma \gamma \mathrm{jj}}^{\mathrm{kin}}$ in a narrow window around $m_{\mathrm{X}}$. The window sizes increase with $m_{\mathrm{X}}$ to account for the increasing experimental resolution from $\Delta m_{\mathrm{X}}= \pm 10 \mathrm{GeV}$ at $m_{\mathrm{X}}=260 \mathrm{GeV}$ to $\Delta m_{\mathrm{X}}={ }_{-20}^{+31} \mathrm{GeV}$ at $m_{\mathrm{X}}=400 \mathrm{GeV}$.

A possible signal can be extracted from data using a simultaneous fit to the $m_{\gamma \gamma}$ and $m_{\mathrm{jj}}$ spectra. The sensitivity to the signal in this search is increased through the $b$ jet energy regression that improves the resolution of the signal in $m_{\mathrm{jj}}$. The background-only PD is a first-order polynomial in the Bernstein basis and a power law in the medium- and high-purity categories, respectively, as shown in Fig. 3, together with their 68\% and 95\% confidence level (C.L.) contours for the selection optimized for the search with $m_{\mathrm{X}}=300 \mathrm{GeV}, 290<m_{\gamma \gamma \mathrm{ji}}^{\mathrm{kin}}<310 \mathrm{GeV}$.

As a cross-check, two alternative signal extraction techniques are tested. In one, a selection is performed in the $m_{\mathrm{jj}}$ spectrum, and the signal extracted in the $m_{\gamma \gamma}$ spectrum. In the other, a selection is performed in the $m_{\mathrm{jj}}$ spectrum and the $m_{\gamma \gamma \mathrm{jj}}$ spectrum is exploited, using a normalization extracted from sidebands in the $m_{\gamma \gamma}$ spectrum. The two procedures give compatible results within the statistical uncertainties.

\section{B. High-mass resonant}

In addition to the requirements in Table I, selections are applied on $m_{\gamma \gamma}$ and $m_{\mathrm{jj}}$, as summarized in Table III.

A possible signal can be extracted from a fit to the $m_{\gamma \gamma \mathrm{jj}}^{\mathrm{kin}}$ distribution for mass points between $320 \leq m_{\gamma \gamma \mathrm{jj}}^{\mathrm{kin}} \leq$ $1200 \mathrm{GeV}$. The background-only PD is a power law for each category, and is seen to well describe the data in Fig. 4. The lower threshold of $320 \mathrm{GeV}$ is chosen to avoid the kinematic turn-on, while still ensuring full containment of signal for the $m_{\mathrm{X}} \geq 400 \mathrm{GeV}$ mass hypotheses. SingleHiggs-boson production is a negligible background in this phase space region, and is absorbed into the parametrization of the nonresonant background.

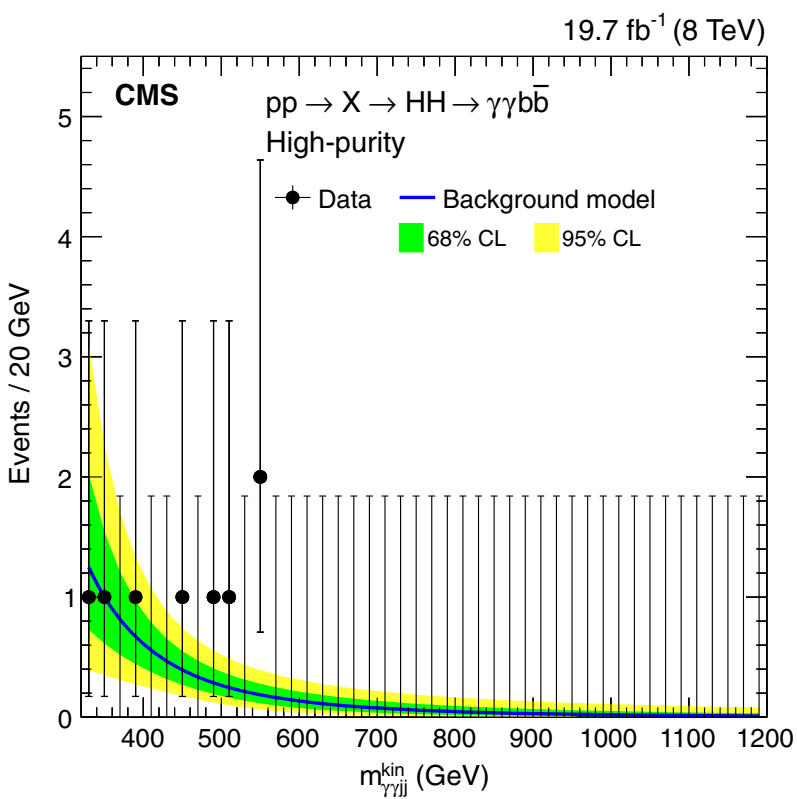

FIG. 4. High-mass resonant analysis: fits to the nonresonant background contribution to the $m_{\gamma \gamma \text { ij }}^{\text {kin }}$ spectrum in the medium- (left) and high-purity (right) events. The fits to the background-only hypothesis are given by the blue curves, along with their 68\% and 95\% C.L. contours. 
TABLE IV. Additional selections applied in the nonresonant searches.

\begin{tabular}{|c|c|c|}
\hline Variable & High purity & Medium purity \\
\hline$\left|\cos \theta_{H H}^{\mathrm{CS}}\right|$ & $<0.9$ & $<0.65$ \\
\hline$m_{\gamma \gamma \gamma \mathrm{j}}^{\mathrm{kin}}$ categorization $(\mathrm{GeV})$ & $<350>350$ & $<350$ \\
\hline
\end{tabular}

\section{Nonresonant}

We apply a selection on $\left|\cos \theta_{H H}^{\mathrm{CS}}\right|$ in the search for nonresonant two-Higgs-boson production. To increase the sensitivity to a large variety of BSM topologies (see examples shown in Fig. 2), an additional categorization is applied in $m_{\gamma \gamma \mathrm{kj}}^{\mathrm{kin}}$. For the SM-like topology in $g g \rightarrow H H$ production, the $m_{\gamma \gamma \mathrm{jj}}^{\mathrm{kin}}$ spectrum peaks roughly at $400 \mathrm{GeV}$, while for $\left|\kappa_{\lambda}\right| \gtrsim 10$ the peak shifts down to the kinematic threshold of $m_{\gamma \gamma \mathrm{jj}}^{\mathrm{kin}} \approx 250 \mathrm{GeV}$. Large values of the $c_{2}$ $\left(\left|c_{2}\right| \approx 3\right)$ parameter usually lead to an opposite effect by shifting the peak in the $m_{\gamma \gamma j \mathrm{j}}^{\mathrm{kin}}$ spectrum above $400 \mathrm{GeV}$. Two categories are defined for $m_{\gamma \gamma \mathrm{jij}}^{\mathrm{kin}}$ smaller or larger than $350 \mathrm{GeV}$, a value optimized for the SM-like search. The details of the selections and categorizations are provided in Table IV.
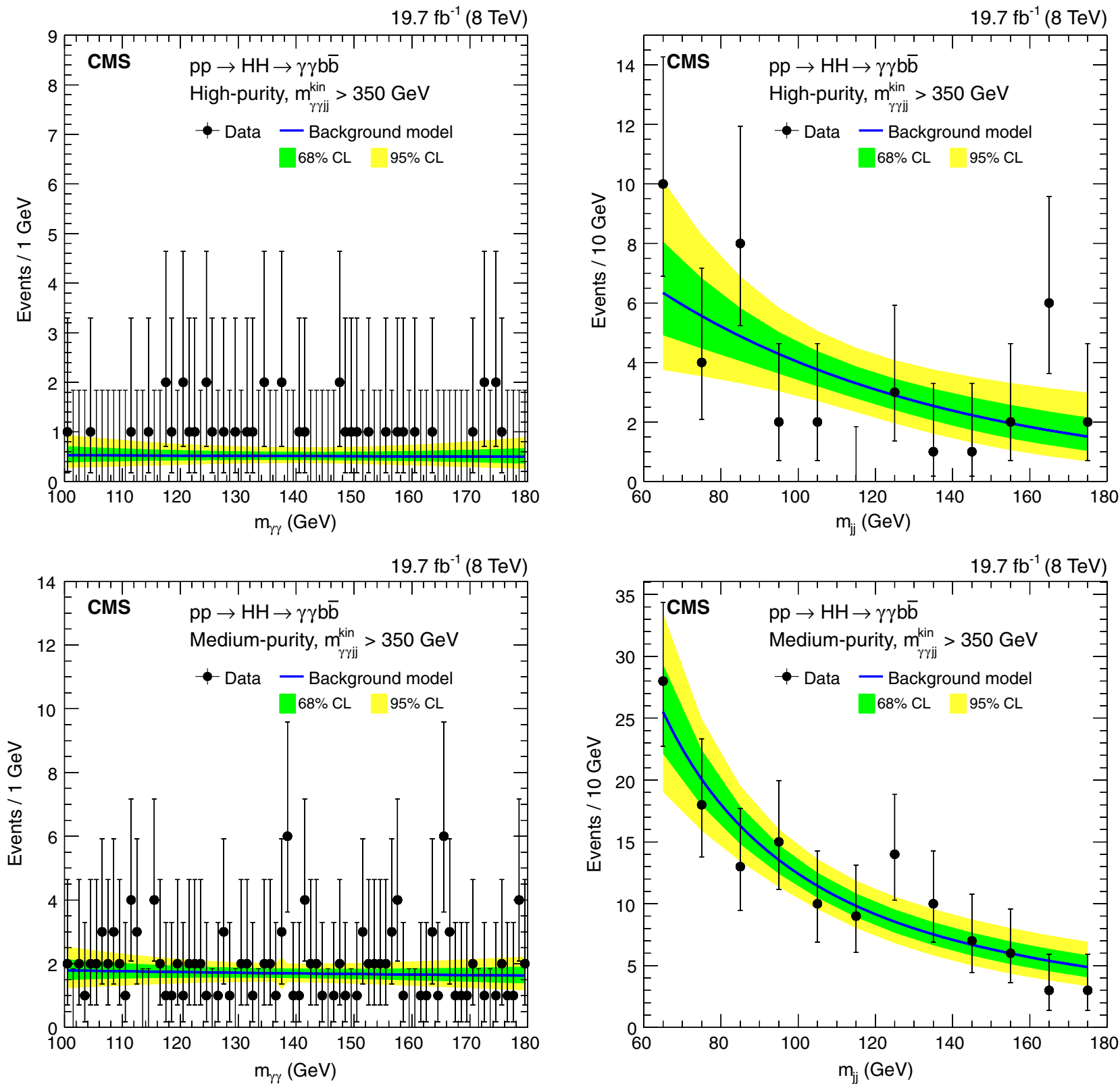

FIG. 5. Nonresonant analysis: fits to the nonresonant background contribution in the high- $m_{\gamma \gamma \mathrm{jij}}^{\text {kin }}$ and high-purity category to the $m_{\gamma \gamma}$ (top left) and $m_{\mathrm{jj}}$ spectra (top right), and similarly for the medium-purity category in the bottom left and bottom right, respectively. The fits to the background-only hypothesis are given by the blue curves, along with their $68 \%$ and $95 \%$ C.L. contours. 

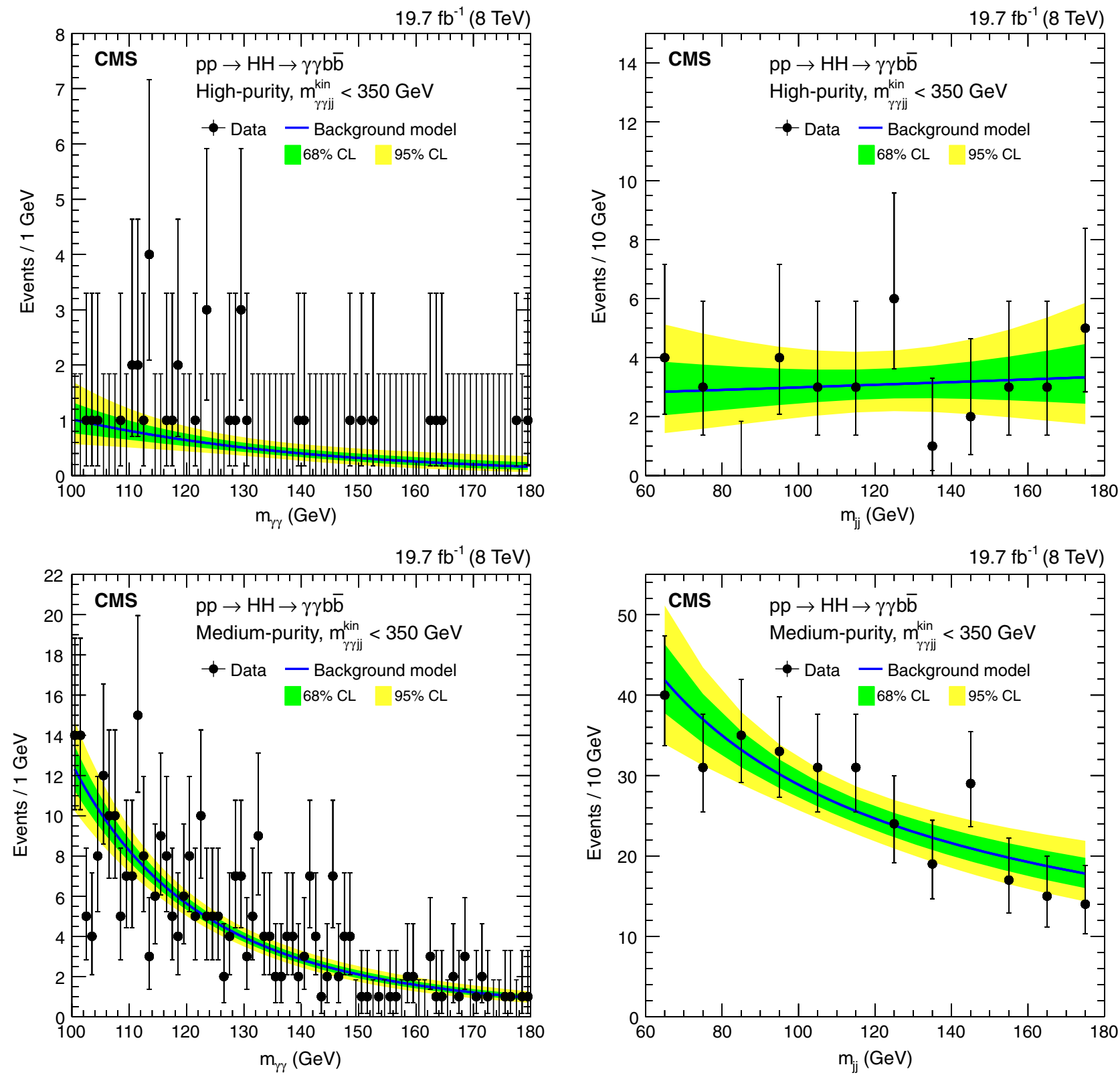

FIG. 6. Nonresonant analysis: fits to the nonresonant background contribution in the low- $m_{\gamma \gamma \mathrm{kj}}^{\mathrm{kin}}$ and high-purity category to the $m_{\gamma \gamma}$ (top-left) and $m_{\mathrm{jj}}$ spectra (top-right), and similarly for the medium-purity category in the bottom left and bottom right, respectively. The fits to the background-only hypothesis are given by the blue curves, along with their $68 \%$ and $95 \%$ C.L. contours.

A possible signal can be extracted using a simultaneous fit to the $m_{\gamma \gamma}$ and $m_{\mathrm{jj}}$ spectra. The backgroundonly PDs are exponentials and power-law expressions for the medium- and high-purity categories, respectively, which agree with the data, as can be seen in Figs. 5 and 6 .

\section{Signal efficiency}

The signal efficiency is a function of the mass hypothesis, as shown in Fig. 7. It is estimated with respect to all events generated in a given signal sample. The efficiency increases as the resonance mass increases from $m_{\mathrm{X}}=260$ to $900 \mathrm{GeV}$ because of higher photon and jet reconstruction efficiencies. The efficiency starts to drop for $m_{\mathrm{X}}>900 \mathrm{GeV}$. At this point, the typical angular distance in the laboratory frame between two $b$ quarks produced in Higgs-boson decay is of the order of the distance parameter $D$ [73]. The minimum in efficiency is observed at $m_{\mathrm{X}}=300 \mathrm{GeV}$. It results from an optimization procedure designed to maximize the overall analysis sensitivity. This procedure chooses an optimal size of $m_{\gamma \gamma \mathrm{jij}}^{\mathrm{kin}}$ window for each $m_{\mathrm{X}}$ hypothesis. For $m_{\mathrm{X}}=300 \mathrm{GeV}$, the background is largest and the resulting $m_{\gamma \gamma j \mathrm{j}}^{\mathrm{kin}}$ window is smallest, inducing a small drop in signal selection efficiency. 


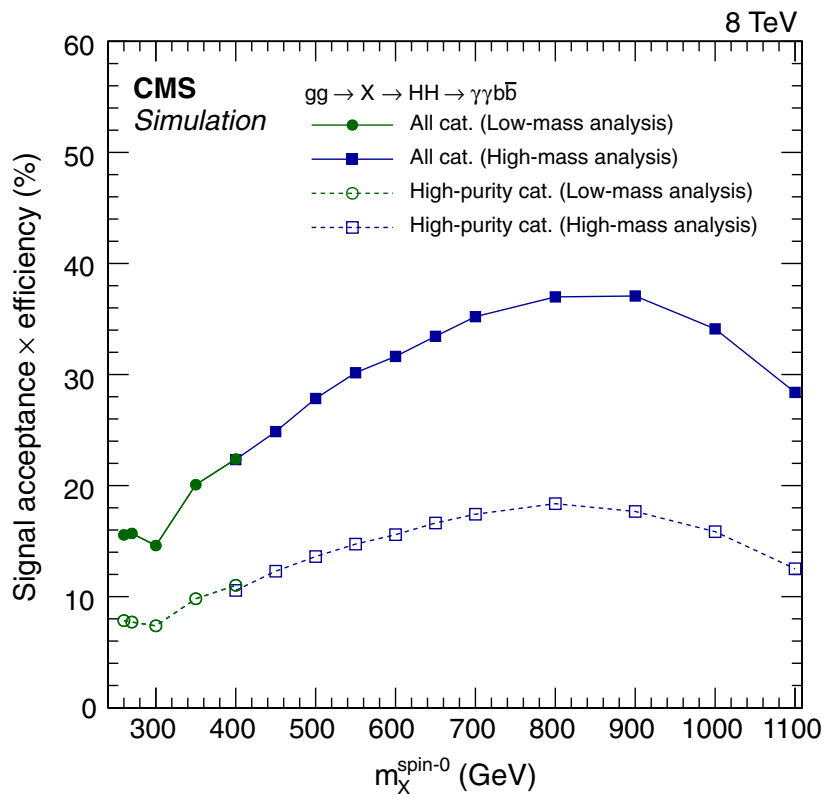

FIG. 7. Resonant signal efficiency for the final selection described in Table I and Sec. V. The efficiency is shown for a spin-0 hypothesis of a radion particle, but is similar for a spin-2 hypothesis of a KK graviton. The error bars associated with statistical uncertainties are smaller than the size of the markers.

Finally, the single and double $b$ tag categories contribute in roughly equal ways to the total efficiency.

Figure 8 provides the efficiencies of selecting the signal events as a function of $\kappa_{\lambda}$ for different values of $\kappa_{t}$ and assuming $c_{2}=0$. The left plot provides efficiencies for $m_{\gamma \gamma \mathrm{jj}}^{\mathrm{kin}}<350 \mathrm{GeV}$ categories and right for $m_{\gamma \gamma \mathrm{jj}}^{\mathrm{kin}}>$ $350 \mathrm{GeV}$ categories. For large absolute values of $\left|\kappa_{\lambda}\right|$ (typically larger than 10) the efficiency is rather flat, while for small values of $\left|\kappa_{\lambda}\right|$ the efficiency in the $m_{\gamma \gamma j \mathrm{j}}^{\mathrm{kin}}<$ $350 \mathrm{GeV} \quad\left(m_{\gamma \gamma \mathrm{jj}}^{\mathrm{kin}}>350 \mathrm{GeV}\right)$ categories is reduced (increased). The change in efficiency is caused by the interference between two-Higgs-boson box diagrams and the Higgs-boson self-coupling channel. The total efficiency in four categories is $\approx 15 \%-30 \%$, depending on the model parameters. This figure illustrates the way that $m_{\gamma \gamma \mathrm{jj}}$ categorization can help separate different nonresonant signal hypotheses.

\section{SYSTEMATIC UNCERTAINTIES}

The analysis defines a likelihood function based on the total PD and the data. The parameters for total signal and for the background-only PD are constrained in the fit to maximize this function. A uniform prior is used to parametrize the background PD. When converting the fitted yields into production cross sections, we use simulations to estimate the selection efficiency for the signal. The difference between the simulation and the data is corrected through scaling factors. The uncertainty in those factors is
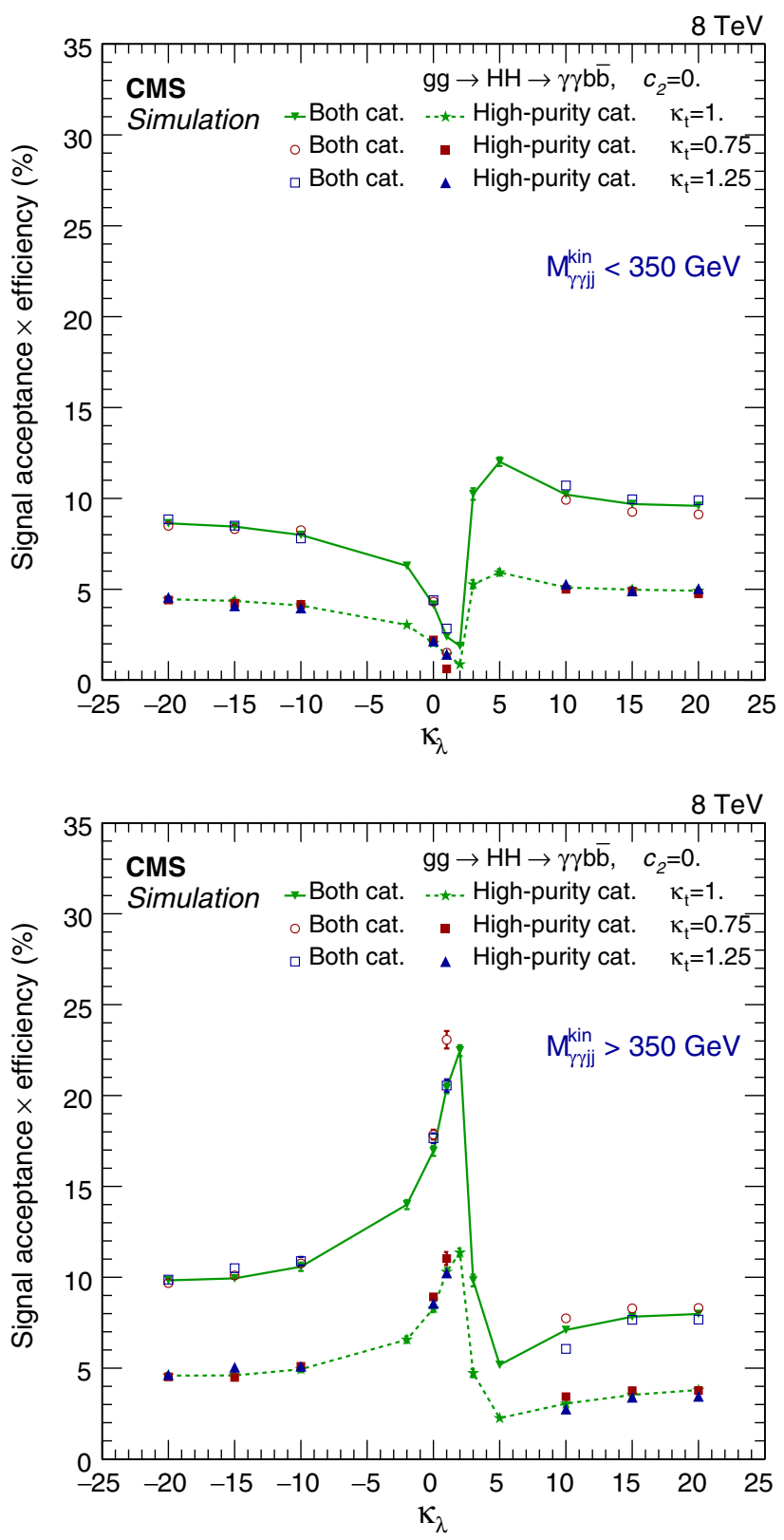

FIG. 8. Signal efficiency for $c_{2}=0$ as a function of $\kappa_{\lambda}$ for different values of $\kappa_{t}$, for the low- $m_{\gamma \gamma \mathrm{jj}}^{\mathrm{kin}}$ region (left) and high- $m_{\gamma \gamma \mathrm{jj}}^{\mathrm{kin}}$ region (right).

taken into account through parameters included in the likelihood function. The nuisance parameters (parameters not of immediate interest) are varied in the fit according to a log-normal probability density function. They can be classified into three categories. The first category contains the uncertainty in the estimation of the integrated luminosity, which is taken as $2.6 \%$ [74]. The second category includes systematic uncertainties that modify the efficiency of signal selection. Finally the third category contains the uncertainties that impact the signal or the Higgs-boson PD. More precisely, the values of the PD parameters are taken from fits to the MC simulation of signal and Higgs-boson 
TABLE V. Summaries of systematic uncertainties. For the normalization uncertainties, the values in the right column indicate the impact on the signal normalization. The uncertainty in the $b$-tagging efficiency is anticorrelated between the $b$ tag categories. The uncertainty in the $m_{\gamma \gamma \mathrm{jj}}^{\mathrm{kin}}$ categorization is anticorrelated between $m_{\gamma \gamma \mathrm{jj}}^{\mathrm{kin}}$ categories for the nonresonant search.

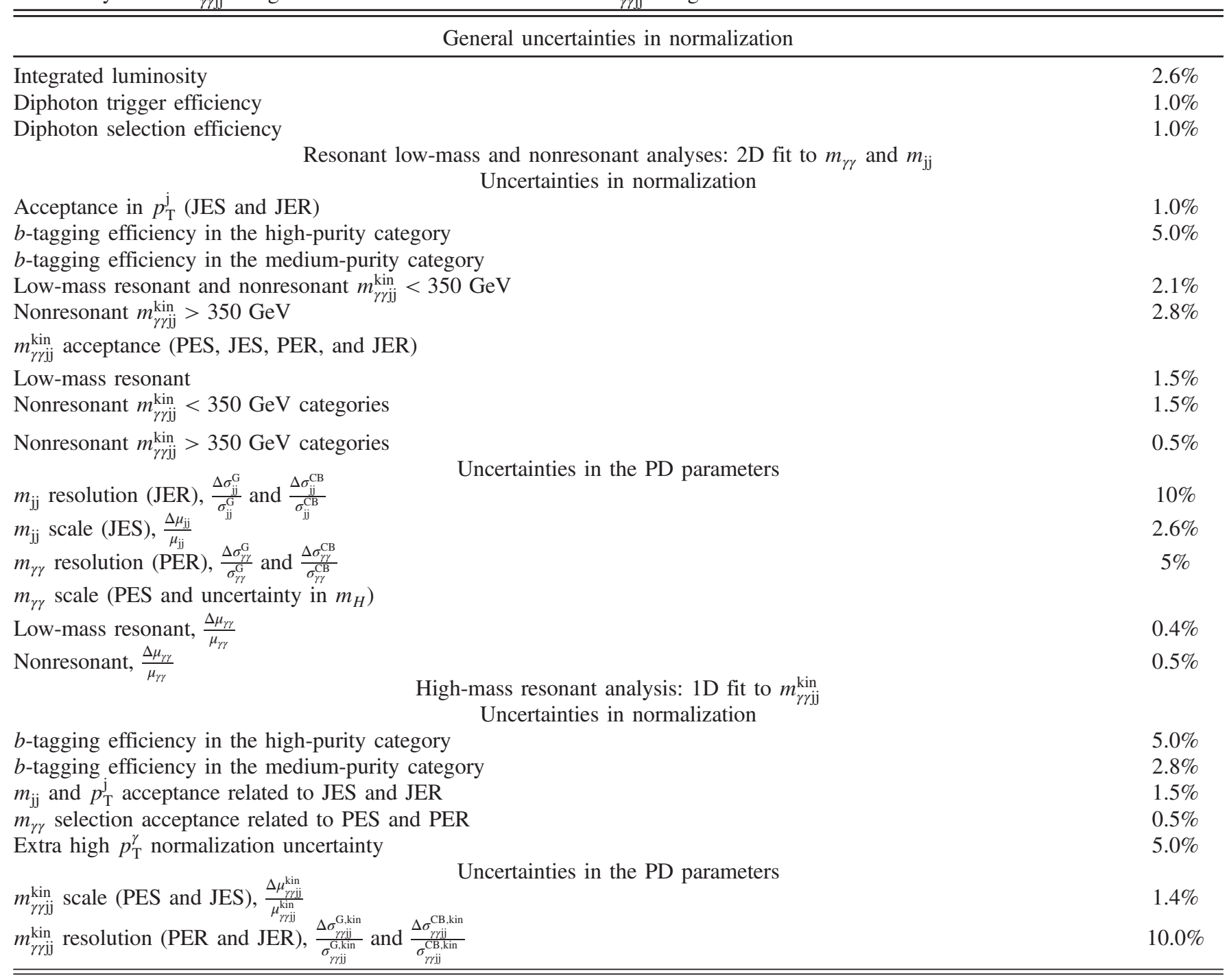

production. The systematic uncertainties affect the mean values and the resolution parameters of the PD, while all other CB parameters are fixed to their best values. The sources of nuisance parameters are described below and their contribution to different categories is presented in Table V.

The photon-related uncertainties are discussed in Ref. [57]. While the photon energy scale (PES) is known at the subpercent level in the region of $p_{\mathrm{T}}^{\gamma}$ characteristic of the SM $H \rightarrow \gamma \gamma$ signal, the uncertainty increases to $1 \%$ for $p_{\mathrm{T}}^{\gamma}>100 \mathrm{GeV}$. The photon energy resolution (PER) is known with a $5 \%$ precision [57]. A $1 \%$ normalization uncertainty is estimated in the offline diphoton selection efficiency and in the trigger efficiency. An additional normalization uncertainty of $5 \%$ is estimated for the high-mass region to account for differences in the $p_{\mathrm{T}}$ spectrum of signal photons and of electrons from $\mathrm{Z} \rightarrow e e$ production used to estimate the quoted uncertainties.
The uncertainty in the jet energy scale (JES) is accounted for by changing the jet response by $1 \%-2 \%$ [68], depending on the kinematics, while the uncertainty in the jet energy resolution (JER) is estimated by changing the jet resolution by $10 \%$ [67]. An additional $1 \%$ uncertainty in the four-body mass accounts for effects in the high-mass region related to the partial overlap between the two $b$ jets from the Higgs-boson decay. The uncertainty in the $b$ tagging efficiency is estimated by changing the $b$-tagging scale factor up and down by 1 standard deviation in each purity category [69]. The related systematic uncertainties are known to be anticorrelated between the two categories.

Theoretical systematic uncertainties are considered for the single-Higgs-boson contribution from SM production, corresponding to the scale dependence of higher-order terms and impact from the choice of proton parton distribution functions (PDF) [36,75]. No theoretical 
uncertainties are assumed on BSM signals. However, there is one exception. We consider the situation where the kinematic properties of the new signal are identical to those of the SM, but the cross section is different (SM-like search). In that case we parametrize the BSM cross section $\sigma_{H H}^{\mathrm{BSM}}$ by the ratio $\mu_{H H}=\sigma_{H H}^{\mathrm{BSM}} / \sigma_{H H}^{\mathrm{SM}}$. When such a search is performed the theoretical uncertainties on $\sigma_{H H}^{\mathrm{SM}}$ are included in the likelihood. Finally, an additional systematic uncertainty of $0.24 \mathrm{GeV}$ is assigned to account for the experimental uncertainty in the Higgs-boson mass [48]. The impact of this uncertainty is comparable to the one from PES.

The analysis is limited by the statistical precision. The systematic uncertainties worsen the expected cross section limits by at most $1.5 \%$ and $3.8 \%$ in the resonant and nonresonant searches, respectively.

\section{RESULTS}

No significant excess is observed over the background expectation in the resonant or nonresonant searches. Upper limits are computed using the modified frequentist approach for confidence levels, taking the profile likelihood as a test statistic [76,77] in the asymptotic approximation. The limits are subsequently compared to theoretical predictions assuming SM branching fractions for Higgs-boson decays.

\section{A. Resonant signal}

The observed and median expected upper limits for all the data at 95\% C.L. are shown in the top of Fig. 9, and at the bottom in a zoomed-in view of the low-mass region. The expected limits range from $1.99 \mathrm{fb}$ for $m_{\mathrm{X}}=310 \mathrm{GeV}$ to $0.39 \mathrm{fb}$ for $m_{\mathrm{X}}=1 \mathrm{TeV}$. At the transition point between the low-mass and high-mass searches, $m_{\mathrm{X}}=400 \mathrm{GeV}$, results with both methods are provided. An improvement of about $20 \%$ is observed from the use of the $2 \mathrm{D}$ model approach with respect to the $1 \mathrm{D}$ analysis.

The result is compared with the cross sections for KKgraviton and radion production in WED models. The tools used to calculate the cross sections for the production of the KK graviton in the bulk and RS1 models are described in Refs. [78,79]. The implementation of the calculations is described in Ref. [80]. In analogy with the Higgs boson, the radion field is predominantly produced through gluon-gluon fusion $[81,82]$. The cross section for radion production is calculated at NLO electroweak and next-to-next-to-leading logarithmic QCD accuracy, using the recipe suggested in Ref. [18]. This recipe consists of multiplying the radion cross section based on the fundamental parameter of the theory, $\Lambda_{\mathrm{R}}$, by a $K$-factor calculated for SM-like Higgs-boson production through gluon-gluon fusion $[36,83]$. The calculations are performed for the SM-like Higgs boson with masses up to $1 \mathrm{TeV}$. We use the CTEQ6L PDF [84] in these calculations. No mixing between a radion and the Higgs boson is considered in this paper.
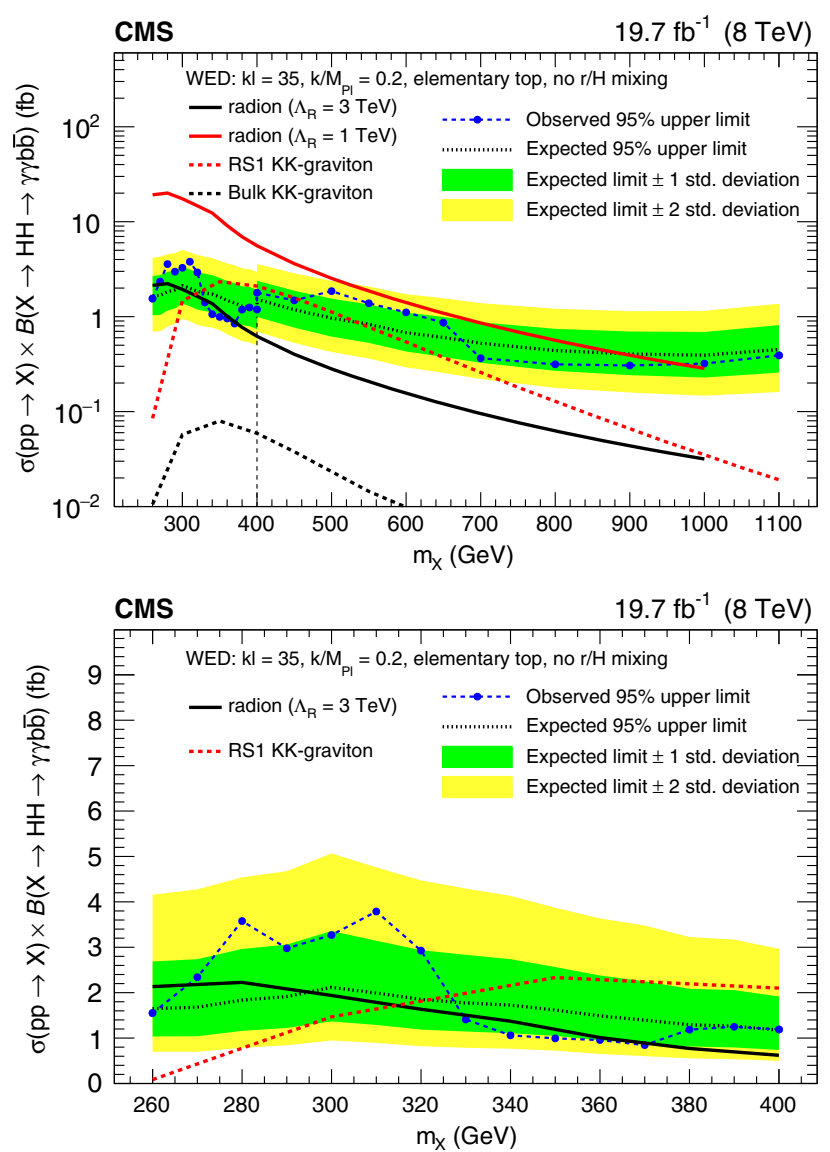

FIG. 9. Observed and expected 95\% C.L. upper limits on the product of the cross section and the branching fraction $\sigma(p p \rightarrow \mathrm{X}) \mathcal{B}(\mathrm{X} \rightarrow H H \rightarrow \gamma \gamma b \bar{b})$ obtained through a combination of the two event categories (top), and in the zoomed view at low mass (bottom). The green and yellow bands represent, respectively, the 1 and 2 standard deviation extensions beyond the expected limit. Also shown are theoretical predictions corresponding to WED models for radions and RS1 KK gravitons. The upper plot with a logarithmic scale for the $y$ axis also provides the prediction for the production cross section of a bulk KK graviton. The vertical dashed line in the upper plot shows the separation between the low-mass and high-mass analyses. The limits for $m_{\mathrm{X}}=400 \mathrm{GeV}$ are shown for both methods.

In Table VI, we summarize the inclusive production cross sections and the branching fractions of the heavy resonances in the theoretical benchmarks we use for interpretation. The absolute values of the production cross sections with $\left(k / \bar{M}_{\mathrm{Pl}}\right)^{2}$ for the KK graviton [22] and scale with $1 / \Lambda_{\mathrm{R}}^{2}$ for the radion [85].

The values for the branching fractions of the resonances in the theory benchmarks do not depend on the fundamental parameters of the theory. The resonance decays have a phase space suppression, related to the mass difference between the resonance and its decay products. In this way, the decay to a Higgs-boson pair is not allowed if $m_{\mathrm{X}}<250 \mathrm{GeV}$ nor to top quark pairs if $m_{\mathrm{X}}<350 \mathrm{GeV}$. In Table VI, we see that the value of the branching fraction 
TABLE VI. Cross section and branching fractions for the benchmark theories used in this paper $[22,85]$. The branching fractions do not depend on $k / \bar{M}_{\mathrm{Pl}}$, or on $\Lambda_{\mathrm{R}}$.

\begin{tabular}{|c|c|c|c|}
\hline \multirow[b]{2}{*}{ Model } & \multicolumn{3}{|c|}{$\sigma(g g \rightarrow \mathrm{X})$} \\
\hline & $m_{\mathrm{X}}(\mathrm{GeV})$ & $(\mathrm{pb})$ & $\mathcal{B}(\mathrm{X} \rightarrow H H)$ \\
\hline \multirow{3}{*}{$\begin{array}{l}\text { RS1 KK graviton } \\
\left(k / \bar{M}_{\mathrm{Pl}}=0.2\right)\end{array}$} & 300 & 2140 & $0.03 \%$ \\
\hline & 500 & 172 & $0.24 \%$ \\
\hline & 1000 & 3.1 & $0.43 \%$ \\
\hline \multirow{3}{*}{$\begin{array}{l}\text { Bulk RS KK graviton } \\
\left(k / \bar{M}_{\mathrm{Pl}}=0.2\right)\end{array}$} & 300 & 0.65 & $0.89 \%$ \\
\hline & 500 & 0.11 & $8.2 \%$ \\
\hline & 1000 & 0.0021 & $9.8 \%$ \\
\hline \multirow{3}{*}{$\begin{array}{l}\text { Radion } \\
\left(\Lambda_{\mathrm{R}}=1 \mathrm{TeV}\right)\end{array}$} & 300 & 20.7 & $32 \%$ \\
\hline & 500 & 3.87 & $25 \%$ \\
\hline & 1000 & 0.46 & $24 \%$ \\
\hline
\end{tabular}

changes with the resonance mass from $m_{\mathrm{X}}=300$ to $m_{\mathrm{X}}=500 \mathrm{GeV}$. The exact pattern of this phenomenon is related to the balance between the different phase space suppressions for decays to $H H$ or to $t \bar{t}$, which depends on the model under consideration.

The analysis excludes a radion with masses below $980 \mathrm{GeV}$ for the radion scale $\Lambda_{\mathrm{R}}=1 \mathrm{TeV}$. The search has also a sensitivity to the presence of a radion with an ultraviolet cutoff $\Lambda_{\mathrm{R}}=3 \mathrm{TeV}$ in the region between 200 and $300 \mathrm{GeV}$.

The difference in total selection efficiency between the spin-0 (radion) and the spin-2 (KK-graviton) models does not exceed $3 \%$. Thus, the same upper limits that are extracted using a radion simulation can be used directly to exclude a KK graviton with masses between 325 and $450 \mathrm{GeV}$, assuming $k / \bar{M}_{\mathrm{Pl}}=0.2$. The analysis is not yet sensitive to the presence of a KK graviton in the bulk scenario with the same parameters.

\section{B. Nonresonant signal}

We consider the kinematic properties for a new signal identical to those of the SM, but with a different cross section. The observed and expected upper limits on SM-like $g g \rightarrow H H \rightarrow \gamma \gamma b \bar{b}$ production are, respectively, 1.85 and $1.56 \mathrm{fb}$. This can be translated into 0.71 and $0.60 \mathrm{pb}$, respectively, for the total $g g \rightarrow H H$ production cross section. The results can also be interpreted in terms of observed and expected limits on the scaling factor $\mu_{H H}<74$ and $<62_{-22}^{+37}$, respectively. This result provides a quantification of the current analysis relative to the SM prediction.

We also interpret the results in the context of Higgsboson anomalous couplings. The cross section for nonresonant two-Higgs-boson production $\sigma_{H H}^{\mathrm{BSM}}$ in this context can be written as a polynomial in the parameters of the theory relative to the SM nonresonant cross section $\sigma_{H H}^{\mathrm{SM}}$ as

$$
\begin{aligned}
\frac{\sigma_{H H}}{\sigma_{H H}^{\mathrm{SM}}}= & A_{1} \kappa_{t}{ }^{4}+A_{2} c_{2}{ }^{2}+A_{3} \kappa_{t}{ }^{2} \kappa_{\lambda}{ }^{2}+\left(A_{6} c_{2}+A_{7} \kappa_{t} \kappa_{\lambda}\right) \kappa_{t}^{2} \\
& +A_{8} \kappa_{t} \kappa_{\lambda} c_{2} .
\end{aligned}
$$

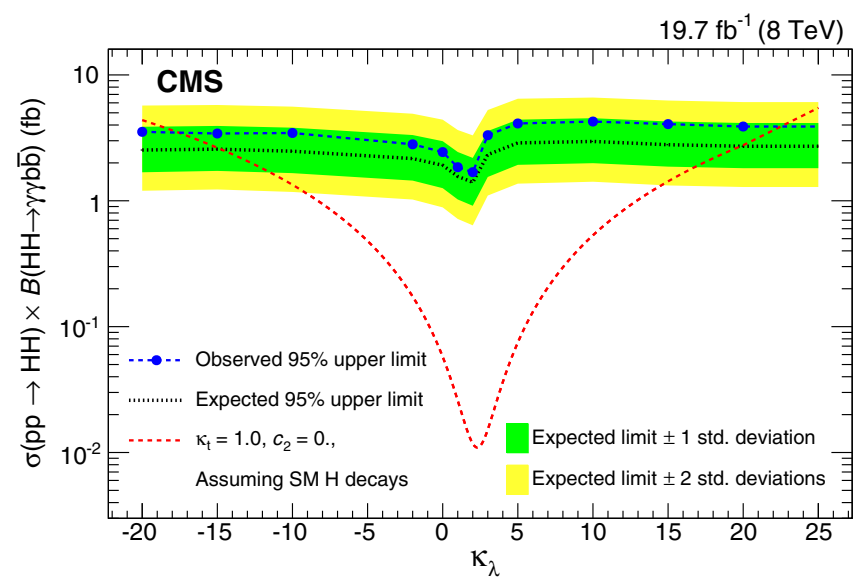

FIG. 10. Observed and expected 95\% C.L. upper limits on the product of cross section and the branching fraction $\sigma(p p \rightarrow H H) \mathcal{B}(H H \rightarrow \gamma \gamma b \bar{b})$ for the nonresonant BSM analysis, performed by changing only $\kappa_{\lambda}$, while keeping all other parameters fixed at the SM predictions.

The numerical coefficients of Eq. (2) can be calculated by fitting cross sections as described in Ref. [86], obtaining thereby $A_{1}=2.19, A_{2}=9.9, \quad A_{3}=0.324, A_{6}=-8.7$, $A_{7}=-1.51$, and $A_{8}=3.0$. Under the assumption that radiative corrections to gluon-gluon fusion of two Higgs bosons do not depend significantly on anomalous interactions [87,88], we normalize $\sigma_{H H}$ such that, when $\kappa_{t}=1$, $\kappa_{\lambda}=1$, and $c_{2}=0$, to the cross section that equals the SM prediction at NNLO in QCD.

In Fig. 10, 95\% C.L. limits on nonresonant cross sections are shown, assuming changes only in the trilinear Higgsboson couplings, with the other parameters fixed to their SM values. All $\kappa_{\lambda}$ values are excluded below -17.5 and above 22.5. These results are obtained by extrapolating the limits between the simulated points, as well as above the highest simulated value of $\kappa_{\lambda}$ using Eq. (2), which relies on the similarity of distributions for signal at large values of $\left|\kappa_{\lambda}\right|[86,89]$, as well as on the behavior of the signal efficiency described in Sec. V D.

Figure 11 shows the 95\% C.L. limits for nonresonant two-Higgs-boson production in the $c_{2}$ and $\kappa_{t}$ planes for different values of $\kappa_{\lambda}$. The specific interference pattern for each combination of parameters produces different exclusion limits for different simulated points of parameter space $[86,89]$. Only discrete values are provided for limits because a linear interpolation between the simulated points could not follow the strong variations due to interference terms. The points in the theoretical phase space excluded by the data are surrounded by small black boxes. Certain combinations of $c_{2}, \kappa_{\lambda}$, or $\kappa_{t}$ parameters can be excluded under the assumption that Higgs bosons have their usual SM branching fractions. For example, we observe that $\left|c_{2}\right| \geq 3$ is disfavored by the data when $\kappa_{\lambda}$ and $\kappa_{t}$ are fixed to $\mathrm{SM}$ values. 


\section{CMS $19.7 \mathrm{fb}^{-1}(8 \mathrm{TeV})$}

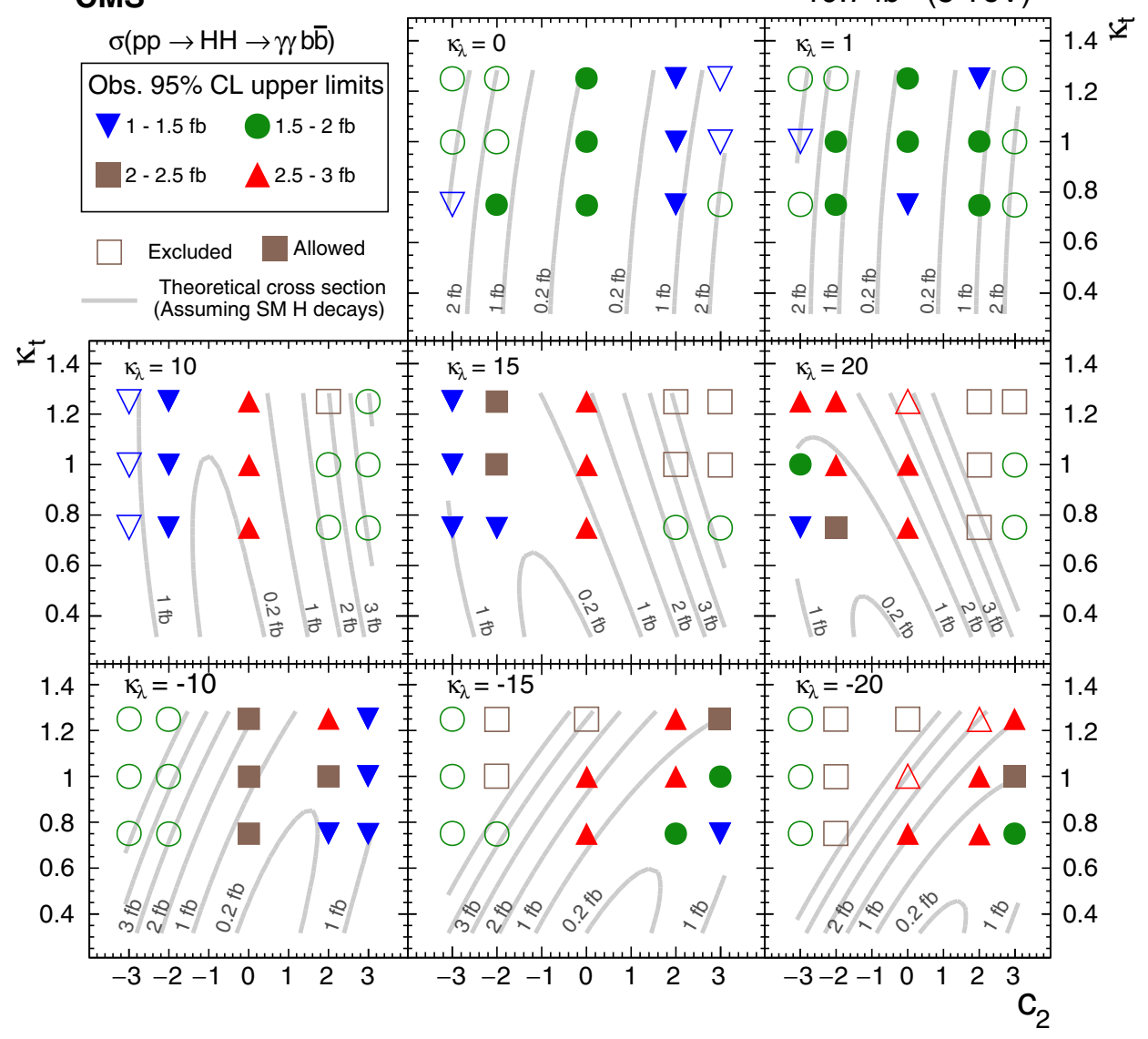

FIG. 11. The observed 95\% C.L. limits for nonresonant two-Higgs-boson production in the $c_{2}$ and $\kappa_{t}$ planes for different values of $\kappa_{\lambda}$. The different markers symbolize the range in which the upper limits in the cross sections are relevant. The results are compared to the theoretical prediction. The gray lines represent contours of equal cross section, as calculated using Eq. (2). The boxed-in cross section markers provide the combination of parameters excluded at $95 \%$ C.L.

\section{SUMMARY}

A search is performed by the CMS collaboration for resonant and nonresonant production of two Higgs bosons in the decay channel $H H \rightarrow \gamma \gamma b \bar{b}$, based on an integrated luminosity of $19.7 \mathrm{fb}^{-1}$ of proton-proton collisions collected at $\sqrt{s}=8 \mathrm{TeV}$. The observations are compatible with expectations from standard model processes. No excess is observed over background predictions.

Resonances are sought in the mass range between 260 and $1100 \mathrm{GeV}$. Upper limits at a 95\% C.L. are extracted on cross sections for the production of new particles decaying to Higgs-boson pairs. The limits are compared to BSM predictions, based on the assumption of the existence of a warped extra dimension. A radion with an ultraviolet cutoff $\Lambda_{\mathrm{R}}=1 \mathrm{TeV}$ is excluded with masses below $980 \mathrm{GeV}$. The search has sensitivity to the presence of a radion with an ultraviolet cutoff $\Lambda_{\mathrm{R}}=3 \mathrm{TeV}$ when its mass lies between 200 and $300 \mathrm{GeV}$. The RS1 KK graviton is excluded with masses between 325 and $450 \mathrm{GeV}$ for $k / \bar{M}_{\mathrm{Pl}}=0.2$. The analysis is not yet sensitive to the presence of a KK graviton in the bulk scenario with the same parameters.
For nonresonant production with SM-like kinematics, a 95\% C.L. upper limit of $1.85 \mathrm{fb}$ is set for the product of the $\mathrm{HH}$ cross section and branching fraction, corresponding to a factor 74 larger than the SM value. When only the trilinear Higgs-boson coupling is changed, values of the self coupling are excluded for $\kappa_{\lambda}<-17$ and $\kappa_{\lambda}>22.5$. The parameter space is also probed for the presence of other anomalous Higgs-boson couplings.

\section{ACKNOWLEDGMENTS}

We are grateful to B. Hespel, F. Maltoni, E. Vryonidou, and $\mathrm{M}$. Zaro for a customized model of the nonresonant signal generation. We congratulate our colleagues in the CERN accelerator departments for the excellent performance of the LHC and thank the technical and administrative staffs at CERN and at other CMS institutes for their contributions to the success of the CMS effort. In addition, we gratefully acknowledge the computing centers and personnel of the Worldwide LHC Computing Grid for delivering so effectively the computing infrastructure essential to our analyses. Finally, we acknowledge the 
enduring support for the construction and operation of the LHC and the CMS detector provided by the following funding agencies: the Austrian Federal Ministry of Science, Research and Economy and the Austrian Science Fund; the Belgian Fonds de la Recherche Scientifique, and Fonds voor Wetenschappelijk Onderzoek; the Brazilian Funding Agencies (CNPq, CAPES, FAPERJ, and FAPESP); the Bulgarian Ministry of Education and Science; CERN; the Chinese Academy of Sciences, Ministry of Science and Technology, and National Natural Science Foundation of China; the Colombian Funding Agency (COLCIENCIAS); the Croatian Ministry of Science, Education and Sport, and the Croatian Science Foundation; the Research Promotion Foundation, Cyprus; the Ministry of Education and Research, Estonian Research Council via Grants No. IUT23-4 and No. IUT23-6 and European Regional Development Fund, Estonia; the Academy of Finland, Finnish Ministry of Education and Culture, and Helsinki Institute of Physics; the Institut National de Physique Nucléaire et de Physique des Particules / CNRS, and Commissariat à l'Énergie Atomique et aux Énergies Alternatives / CEA, France; the Bundesministerium für Bildung und Forschung, Deutsche Forschungsgemeinschaft, and Helmholtz-Gemeinschaft Deutscher Forschungszentren, Germany; the General Secretariat for Research and Technology, Greece; the National Scientific Research Foundation, and National Innovation Office, Hungary; the Department of Atomic Energy and the Department of Science and Technology, India; the Institute for Studies in Theoretical Physics and Mathematics, Iran; the Science Foundation, Ireland; the Istituto Nazionale di Fisica Nucleare, Italy; the Ministry of Science, ICT and Future Planning, and National Research Foundation (NRF), Republic of Korea; the Lithuanian Academy of Sciences; the Ministry of Education, and University of Malaya (Malaysia); the Mexican Funding Agencies (CINVESTAV, CONACYT, SEP, and UASLPFAI); the Ministry of Business, Innovation and Employment, New Zealand; the Pakistan Atomic Energy Commission; the Ministry of Science and Higher Education and the National Science Centre, Poland; the Fundação para a Ciência e a Tecnologia, Portugal; JINR, Dubna; the Ministry of Education and Science of the Russian Federation, the Federal Agency of Atomic
Energy of the Russian Federation, Russian Academy of Sciences, and the Russian Foundation for Basic Research; the Ministry of Education, Science and Technological Development of Serbia; the Secretaría de Estado de Investigación, Desarrollo e Innovación and Programa Consolider-Ingenio 2010, Spain; the Swiss Funding Agencies (ETH Board, ETH Zurich, PSI, SNF, UniZH, Canton Zurich, and Swiss State Secretariat for Education and Research (Education, Research, and Innovation: SERI)); the Ministry of Science and Technology, Taipei; the Thailand Center of Excellence in Physics, the Institute for the Promotion of Teaching Science and Technology of Thailand, Special Task Force for Activating Research and the National Science and Technology Development Agency of Thailand; the Scientific and Technical Research Council of Turkey, and Turkish Atomic Energy Authority; the National Academy of Sciences of Ukraine, and State Fund for Fundamental Researches, Ukraine; the Science and Technology Facilities Council, United Kingdom; the U.S. Department of Energy, and the U.S. National Science Foundation. Individuals have received support from the Marie-Curie program and the European Research Council and EPLANET (European Union); the Leventis Foundation; the A. P. Sloan Foundation; the Alexander von Humboldt Foundation; the Belgian Federal Science Policy Office; the Fonds pour la Formation à la Recherche dans l'Industrie et dans l'Agriculture (FRIA-Belgium); the Agentschap voor Innovatie door Wetenschap en Technologie (IWTBelgium); the Ministry of Education, Youth and Sports (MEYS) of the Czech Republic; the Council of Science and Industrial Research, India; the HOMING PLUS program of the Foundation for Polish Science, cofinanced from European Union, Regional Development Fund; the OPUS program of the National Science Center (Poland); the Compagnia di San Paolo (Torino); MIUR project Grant No. 20108T4XTM (Italy); the Thalis and Aristeia programs cofinanced by EU-ESF and the Greek NSRF; the National Priorities Research Program by Qatar National Research Fund; the Rachadapisek Sompot Fund for Postdoctoral Fellowship, Chulalongkorn University (Thailand); the Chulalongkorn Academic into its 2nd Century Project Advancement Project (Thailand); and the Welch Foundation, Contract No. C-1845.
[1] S. Chatrchyan et al. (CMS Collaboration), Observation of a new boson at a mass of $125 \mathrm{GeV}$ with the CMS experiment at the LHC, Phys. Lett. B 716, 30 (2012).

[2] ATLAS Collaboration, Observation of a new particle in the search for the standard model Higgs boson with the ATLAS detector at the LHC, Phys. Lett. B 716, 1 (2012).
[3] D. de Florian and J. Mazzitelli, Higgs Boson Pair Production at Next-to-Next-to-Leading Order in QCD, Phys. Rev. Lett. 111, 201801 (2013).

[4] S. Dawson, S. Dittmaier, and M. Spira, Neutral Higgs boson pair production at hadron colliders: QCD corrections, Phys. Rev. D 58, 115012 (1998). 
[5] J. Baglio, A. Djouadi, R. Gröber, M. M. Mühlleitner, J. Quevillon, and M. Spira, The measurement of the Higgs self-coupling at the LHC: theoretical status, J. High Energy Phys. 04 (2013) 151.

[6] S. Dawson, A. Ismail, and I. Low, What's in the loop? The anatomy of double Higgs production, Phys. Rev. D 91, 115008 (2015).

[7] Z. Heng, L. Shang, Y. Zhang, and J. Zhu, Pair production of $125 \mathrm{GeV}$ Higgs boson in the SM extension with color-octet scalars at the LHC, J. High Energy Phys. 02 (2014) 083.

[8] R. Gröber and M. Mühlleitner, Composite Higgs boson pair production at the LHC, J. High Energy Phys. 06 (2011) 020.

[9] M. Moretti, S. Moretti, F. Piccinini, R. Pittau, and A. D. Polosa, Higgs boson self-couplings at the LHC as a probe of extended Higgs sectors, J. High Energy Phys. 02 (2005) 024.

[10] L. Randall and R. Sundrum, Large Mass Hierarchy from a Small Extra Dimension, Phys. Rev. Lett. 83, 3370 (1999).

[11] W. D. Goldberger and M. B. Wise, Modulus Stabilization with Bulk Fields, Phys. Rev. Lett. 83, 4922 (1999).

[12] O. DeWolfe, D. Z. Freedman, S. S. Gubser, and A. Karch, Modeling the fifth dimension with scalars and gravity, Phys. Rev. D 62, 046008 (2000).

[13] C. Csáki, M. Graesser, L. Randall, and J. Terning, Cosmology of brane models with radion stabilization, Phys. Rev. D 62, 045015 (2000).

[14] H. Davoudiasl, J. L. Hewett, and T. G. Rizzo, Phenomenology of the Randall-Sundrum Gauge Hierarchy Model, Phys. Rev. Lett. 84, 2080 (2000).

[15] C. Csáki, M. L. Graesser, and G. D. Kribs, Radion dynamics and electroweak physics, Phys. Rev. D 63, 065002 (2001).

[16] K. Agashe, H. Davoudiasl, G. Perez, and A. Soni, Warped gravitons at the CERN LHC and beyond, Phys. Rev. D 76, 036006 (2007).

[17] A. Liam Fitzpatrick, Jared Kaplan, Lisa Randall, and LianTao Wang, Searching for the Kaluza-Klein graviton in bulk RS models, J. High Energy Phys. 09 (2007) 013.

[18] G. F. Giudice, R. Rattazzi, and J. D. Wells, Graviscalars from higher-dimensional metrics and curvature-Higgs mixing, Nucl. Phys. B595, 250 (2001).

[19] D. Dominici, B. Grzadkowski, J. F. Gunion, and M. Toharia, The scalar sector of the Randall-Sundrum model, Nucl. Phys. B671, 243 (2003).

[20] I. Antoniadis and R. Sturani, Higgs graviscalar mixing in type I string theory, Nucl. Phys. B631, 66 (2002).

[21] N. Desai, U. Maitra, and B. Mukhopadhyaya, An updated analysis of radion-Higgs mixing in the light of LHC data, J. High Energy Phys. 10 (2013) 093.

[22] A. Oliveira and R. Rosenfeld, Graviscalars from higherdimensional metrics and curvature-Higgs mixing, Phys. Lett. B 702, 201 (2011).

[23] D. O'Connell, M. J. Ramsey-Musolf, and M. B. Wise, Minimal extension of the standard model scalar sector, Phys. Rev. D 75, 037701 (2007).

[24] G. C. Branco, P. M. Ferreira, L. Lavoura, M. N. Rebelo, M. Sher, and J. P. Silva, Theory and phenomenology of twoHiggs-doublet modeld, Phys. Rep. 516, 1 (2012).

[25] A. Djouadi, The anatomy of electroweak symmetry breaking Tome II: the Higgs bosons in the minimal supersymmetric model, Phys. Rep. 459, 1 (2008).
[26] R. Barbieri, D. Buttazzo, K. Kannike, F. Sala, and A. Tesi, One or more Higgs bosons?, Phys. Rev. D 88, 055011 (2013).

[27] H. Georgi and M. Machacek, Doubly charged Higgs bosons, Nucl. Phys. B262, 463 (1985).

[28] E. W. Nigel Glover and J. J. van der Bij, Higgs boson pair production via gluon fusion, Nucl. Phys. B309, 282 (1988).

[29] T. Plehn, M. Spira, and P. M. Zerwas, Pair production of neutral Higgs particles in gluon-gluon collisions, Nucl. Phys. B479, 46 (1996).

[30] A. Djouadi, W. Kilian, M. Mühlleitner, and P. M. Zerwas, Production of neutral Higgs boson pairs at LHC, Eur. Phys. J. C 10, 45 (1999).

[31] U. Baur, T. Plehn, and D. L. Rainwater, Probing the Higgs self-coupling at hadron colliders using rare decays, Phys. Rev. D 69, 053004 (2004).

[32] A. Pierce, J. Thaler, and L.-T. Wang, Disentangling dimension six operators through di-Higgs boson production, J. High Energy Phys. 05 (2007) 070.

[33] K. Nishiwaki, S. Niyogi, and A. Shivaji, $t \bar{t} H$ anomalous coupling in double Higgs production, J. High Energy Phys. 04 (2014) 011.

[34] F. Goertz, A. Papaefstathiou, L. L. Yang, and J. Zurita, Higgs boson pair production in the $D=6$ extension of the SM, J. High Energy Phys. 04 (2015) 167.

[35] N. Liu, S. Hu, B. Yang, and J. Han, Impact of top-Higgs couplings on di-Higgs production at future colliders, J. High Energy Phys. 01 (2015) 008.

[36] S Heinemeyer et al., CERN Report No. CERN-2013-004, 2013.

[37] J. C. Collins and D. E. Soper, Angular distribution of dileptons in high-energy hadron collisions, Phys. Rev. D 16, 2219 (1977).

[38] ATLAS Collaboration, Search For Higgs Boson Pair Production in the $\gamma \gamma b \bar{b}$ Final State Using pp Collision Data at $\sqrt{s}=8 \mathrm{TeV}$ from the ATLAS Detector, Phys. Rev. Lett. 114, 081802 (2015).

[39] ATLAS Collaboration, Search for Higgs boson pair production in the $b \bar{b} b \bar{b}$ final state from pp collisions at $\sqrt{s}=$ $8 \mathrm{TeV}$ with the ATLAS detector, Eur. Phys. J. C 75, 412 (2015).

[40] ATLAS Collaboration, Searches for Higgs boson pair production in the $h h \rightarrow b \bar{b} \tau \tau, \gamma \gamma W W^{*}, \gamma \gamma b \bar{b}, b \bar{b} b \bar{b}$ channels with the ATLAS detector, Phys. Rev. D 92, 092004 (2015).

[41] CMS Collaboration, Searches for heavy Higgs bosons in two-Higgs-doublet models and for $t \rightarrow c h$ decay using multilepton and diphoton final states in pp collisions at 8 TeV, Phys. Rev. D 90, 112013 (2014).

[42] CMS Collaboration, Search for resonant pair production of Higgs bosons decaying to two bottom quark-antiquark pairs in proton-proton collisions at $8 \mathrm{TeV}$, Phys. Lett. B 749, 560 (2015).

[43] CMS Collaboration, Search for heavy resonances decaying to two Higgs bosons in final states containing four b quarks, Eur. Phys. J. C 76, 371 (2016).

[44] CMS Collaboration, Searches for a heavy scalar boson $\mathrm{H}$ decaying to a pair of $125 \mathrm{GeV}$ Higgs bosons hh or for a heavy pseudoscalar boson A decaying to Zh, 
in the final states with $h \rightarrow \tau \tau$, Phys. Lett. B 755, 217 (2016).

[45] CMS Collaboration, The CMS experiment at the CERN LHC, J. Instrum. 3, S08004 (2008).

[46] J. Alwall, R. Frederix, S. Frixione, V. Hirschi, F. Maltoni, O. Mattelaer, H. S. Shao, T. Stelzer, P. Torrielli, and M. Zaro, The automated computation of tree-level and next-toleading order differential cross sections, and their matching to parton shower simulations, J. High Energy Phys. 07 (2014) 079.

[47] T. Sjöstrand, S. Mrenna, and P. Skands, PYTHIA 6.4 physics and manual, J. High Energy Phys. 05 (2006) 026.

[48] ATLAS and CMS Collaborations, Combined Measurement of the Higgs Boson Mass in $p p$ Collisions at $\sqrt{s}=7$ and $8 \mathrm{TeV}$ with the ATLAS and CMS Experiments, Phys. Rev. Lett. 114, 191803 (2015).

[49] CMS Collaboration, Precise determination of the mass of the Higgs boson and tests of compatibility of its couplings with the standard model predictions using proton collisions at 7 and 8 TeV, Eur. Phys. J. C 75, 212 (2015).

[50] T. Corbett, O. J. P. Eboli, D. Goncalves, J. Gonzalez-Fraile, T. Plehn, and M. Rauch, The nonlinear Higgs legacy of the LHC run I, arXiv:1511.08188.

[51] T. Gleisberg, S. Hoeche, F. Krauss, M. Schonherr, S. Schumann, F. Siegert, and J. Winter, Event generation with SHERPA 1.1, J. High Energy Phys. 02 (2009) 007.

[52] Paolo Nason, A new method for combining NLO QCD with shower Monte Carlo algorithms, J. High Energy Phys. 11 (2004) 040.

[53] S. Frixione, P. Nason, and C. Oleari, Matching NLO QCD computations with parton shower simulations: the POWHEG method, J. High Energy Phys. 11 (2007) 070.

[54] S. Alioli, P. Nason, C. Oleari, and E. Re, A general framework for implementing NLO calculations in shower Monte Carlo programs: the POWHEG BOX, J. High Energy Phys. 06 (2010) 043.

[55] S. Agostinelli et al. (GEANT4 Collaboration), GEANT4-a simulation toolkit, Nucl. Instrum. Methods Phys. Res., Sect. A 506, 250 (2003).

[56] J. Allison et al., Geant4 developments and applications, IEEE Trans. Nucl. Sci. 53, 270 (2006).

[57] CMS Collaboration, Observation of the diphoton decay of the Higgs boson and measurement of its properties, Eur. Phys. J. C 74, 3076 (2014).

[58] CMS Collaboration, CMS Report No. CMS-PAS-EGM-10005, 2010, http://cds.cern.ch/record/1279143?ln=en.

[59] CMS Collaboration, CMS Report No. CMS-PAS-EGM-10006, 2011, http://cds.cern.ch/record/1324545.

[60] CMS Collaboration, Energy calibration and resolution of the CMS electromagnetic calorimeter in $p p$ collisions at $\sqrt{s}=7 \mathrm{TeV}$, J. Instrum. 8, P09009 (2013).

[61] CMS Collaboration, Search for the standard model Higgs boson produced in association with a $\mathrm{W}$ or a $\mathrm{Z}$ boson and decaying to bottom quarks, Phys. Rev. D 89, 012003 (2014).

[62] CMS Collaboration, CMS Report No. CMS-PAS-PFT-09001, 2009, http://cdsweb.cern.ch/record/1194487.
[63] CMS Collaboration, CMS Report No. CMS-PAS-PFT-10001, 2010, http://cdsweb.cern.ch/record/1247373.

[64] M. Cacciari, G. P. Salam, and G. Soyez, The anti- $k_{t}$ jet clustering algorithm, J. High Energy Phys. 04 (2008) 063.

[65] M. Cacciari and G. P. Salam, Pileup subtraction using jet areas, Phys. Lett. B 659, 119 (2008).

[66] M. Cacciari, G. P. Salam, and G. Soyez, FastJet user manual, Eur. Phys. J. C 72, 1896 (2012).

[67] CMS Collaboration, Determination of jet energy calibration and transverse momentum resolution in CMS, J. Instrum. 6, P11002 (2011).

[68] CMS Collaboration, CMS, Report No. CMS-DP-2013-011, 2013, https://cds.cern.ch/record/1545350.

[69] CMS Collaboration, Identification of b-quark jets with the CMS experiment, J. Instrum. 8, P04013 (2013).

[70] CMS Collaboration, Search for a standard-model-like Higgs boson with a mass in the range 145 to $1000 \mathrm{GeV}$ at the LHC, Eur. Phys. J. C 73, 2469 (2013).

[71] CMS Collaboration, Search for a fermiophobic Higgs boson in $p p$ collisions at $\sqrt{s}=7 \mathrm{TeV}$, J. High Energy Phys. 09 (2012) 111.

[72] M. J. Oreglia, SLAC Report No. SLAC-R-236.

[73] M. Gouzevitch, A. Oliveira, J. Rojo, R. Rosenfeld, G. P. Salam, and V. Sanz, Scale-invariant resonance tagging in multijet events and new physics in Higgs pair production, J. High Energy Phys. 07 (2013) 148.

[74] CMS Collaboration, CMS, Report No. CMS-PAS-LUM-13001, 2013, http://cds.cern.ch/record/1598864.

[75] S. Dittmaier et al. (LHC Higgs Cross Section Working Group), CERN Report No. CERN-2011-002, 2011.

[76] A. L. Read, Presentation of search results: the $\mathrm{CL}_{s}$ technique, J. Phys. G 28, 2693 (2002).

[77] T. Junk, Confidence level computation for combining searches with small statistics, Nucl. Instrum. Methods Phys. Res., Sect. A 434, 435 (1999).

[78] K. Agashe et al., in Community Summer Study 2013: Snowmass on the Mississippi (CSS2013) Minneapolis, 2013 (2013), https://inspirehep.net/record/1222762.

[79] P. de Aquino, K. Hagiwara, Q. Li, and F. Maltoni, Simulating graviton production at hadron colliders, J. High Energy Phys. 06 (2011) 132.

[80] A. Oliveira, Gravity particles from warped extra dimensions, a review. Part I-KK graviton, arXiv:1404.0102.

[81] U. Mahanta and A. Datta, Search prospects of light stabilized radions at Tevatron and LHC, Phys. Lett. B 483, 196 (2000).

[82] H. Davoudiasl, J. L. Hewett, and T. G. Rizzo, Experimental probes of localized gravity: on and off the wall, Phys. Rev. D 63, 075004 (2001).

[83] S. Catani, D. de Florian, M. Grazzini, and P. Nason, Soft gluon resummation for Higgs boson production at hadron colliders, J. High Energy Phys. 07 (2003) 028.

[84] P. M. Nadolsky, H.-L. Lai, Q.-H. Cao, J. Huston, J. Pumplin, D. Stump, W.-K Tung, and C.-P. Yuan, Implications of CTEQ global analysis for collider observables, Phys. Rev. D 78, 013004 (2008).

[85] V. Barger and M. Ishida, Randall-Sundrum reality at the LHC, Phys. Lett. B 709, 185 (2012). 
[86] A. Carvalho, M. Dall'Osso, T. Dorigo, F. Goertz, C. A. Gottardo, and M. Tosi, Higgs pair production: choosing benchmarks with cluster analysis, J. High Energy Phys. 04 (2016) 126.

[87] R. Gröber, M. Mühlleitner, M. Spira, and J. Streicher, NLOQCD corrections to Higgs pair production including dimension-6 operators, J. High Energy Phys. 09 (2015) 092.
[88] D. de Florian and J. Mazzitelli, Higgs pair production at next-to-next-to-leading logarithmic accuracy at the LHC, J. High Energy Phys. 09 (2015) 053.

[89] A. Carvalho, M. Dall'Osso, P. de Castro Manzano, T. Dorigo, F. Goertz, M. Gouzevich, and M. Tosi, Analytical parametrization and shape classification of anomalous $\mathrm{HH}$ production in the EFT approach, arXiv:1608.06578.

V. Khachatryan, ${ }^{1}$ A. M. Sirunyan, ${ }^{1}$ A. Tumasyan, ${ }^{1}$ W. Adam, ${ }^{2}$ E. Asilar, ${ }^{2}$ T. Bergauer, ${ }^{2}$ J. Brandstetter, ${ }^{2}$ E. Brondolin, ${ }^{2}$ M. Dragicevic, ${ }^{2}$ J. Erö, ${ }^{2}$ M. Flechl, ${ }^{2}$ M. Friedl, ${ }^{2}$ R. Frühwirth, ${ }^{2, b}$ V. M. Ghete, ${ }^{2}$ C. Hartl, ${ }^{2}$ N. Hörmann, ${ }^{2}$ J. Hrubec, ${ }^{2}$ M. Jeitler, ${ }^{2, \mathrm{~b}}$ A. König, ${ }^{2}$ M. Krammer, ${ }^{2, \mathrm{~b}}$ I. Krätschmer, ${ }^{2}$ D. Liko, ${ }^{2}$ T. Matsushita, ${ }^{2}$ I. Mikulec, ${ }^{2}$ D. Rabady, ${ }^{2}$ N. Rad, ${ }^{2}$ B. Rahbaran, ${ }^{2}$ H. Rohringer, ${ }^{2}$ J. Schieck, ${ }^{2, b}{ }^{2}$. Strauss, ${ }^{2}$ W. Treberer-Treberspurg, ${ }^{2}$ W. Waltenberger, ${ }^{2}$ C.-E. Wulz, ${ }^{2, b}$ V. Mossolov, ${ }^{3}$ N. Shumeiko, ${ }^{3}$ J. Suarez Gonzalez, ${ }^{3}$ S. Alderweireldt, ${ }^{4}$ T. Cornelis, ${ }^{4}$ E. A. De Wolf, ${ }^{4}$ X. Janssen, ${ }^{4}$ A. Knutsson, ${ }^{4}$ J. Lauwers, ${ }^{4}$ S. Luyckx ${ }^{4}$ M. Van De Klundert, ${ }^{4}$ H. Van Haevermaet, ${ }^{4}$ P. Van Mechelen, ${ }^{4}$ N. Van Remortel, ${ }^{4}$ A. Van Spilbeeck, ${ }^{4}$ S. Abu Zeid, ${ }^{5}$ F. Blekman, ${ }^{5}$ J. D'Hondt,${ }^{5}$ N. Daci, ${ }^{5}$ I. De Bruyn, ${ }^{5}$ K. Deroover, ${ }^{5}$ N. Heracleous, ${ }^{5}$ J. Keaveney, ${ }^{5}$ S. Lowette, ${ }^{5}$ S. Moortgat, ${ }^{5}$ L. Moreels, ${ }^{5}$ A. Olbrechts, ${ }^{5}$ Q. Python, ${ }^{5}$ D. Strom, ${ }^{5}$ S. Tavernier, ${ }^{5}$

W. Van Doninck, ${ }^{5}$ P. Van Mulders, ${ }^{5}$ I. Van Parijs, ${ }^{5}$ H. Brun, ${ }^{6}$ C. Caillol, ${ }^{6}$ B. Clerbaux, ${ }^{6}$ G. De Lentdecker, ${ }^{6}$ G. Fasanella, ${ }^{6}$ L. Favart, ${ }^{6}$ R. Goldouzian, ${ }^{6}$ A. Grebenyuk, ${ }^{6}$ G. Karapostoli, ${ }^{6}$ T. Lenzi, ${ }^{6}$ A. Léonard, ${ }^{6}$ T. Maerschalk, ${ }^{6}$ A. Marinov ${ }^{6}$ A. Randle-conde, ${ }^{6}$ T. Seva, ${ }^{6}$ C. Vander Velde, ${ }^{6}$ P. Vanlaer, ${ }^{6}$ R. Yonamine, ${ }^{6}$ F. Zenoni, ${ }^{6}$ F. Zhang, ${ }^{6, c}$ L. Benucci, ${ }^{7}$ A. Cimmino, ${ }^{7}$ S. Crucy, ${ }^{7}$ D. Dobur, ${ }^{7}$ A. Fagot, ${ }^{7}$ G. Garcia, ${ }^{7}$ M. Gul, ${ }^{7}$ J. Mccartin, ${ }^{7}$ A. A. Ocampo Rios, ${ }^{7}$ D. Poyraz, ${ }^{7}$ D. Ryckbosch, ${ }^{7}$ S. Salva, ${ }^{7}$ R. Schöfbeck, ${ }^{7}$ M. Sigamani, ${ }^{7}$ M. Tytgat,${ }^{7}$ W. Van Driessche, ${ }^{7}$ E. Yazgan, ${ }^{7}$ N. Zaganidis, ${ }^{7}$ C. Beluffi, ${ }^{8, \mathrm{~d}}$ O. Bondu, ${ }^{8}$ S. Brochet, ${ }^{8}$ G. Bruno, ${ }^{8}$ A. Caudron, ${ }^{8}$ L. Ceard,${ }^{8}$ S. De Visscher, ${ }^{8}$ C. Delaere,${ }^{8}$ M. Delcourt, ${ }^{8}$ L. Forthomme, ${ }^{8}$ B. Francois, ${ }^{8}$ A. Giammanco,${ }^{8}$ A. Jafari, ${ }^{8}$ P. Jez,${ }^{8}$ M. Komm,${ }^{8}$ V. Lemaitre, ${ }^{8}$ A. Magitteri, ${ }^{8}$ A. Mertens, ${ }^{8}$ M. Musich ${ }^{8}$ C. Nuttens,${ }^{8}$ K. Piotrzkowski, ${ }^{8}$ L. Quertenmont, ${ }^{8}$ M. Selvaggi, ${ }^{8}$ M. Vidal Marono, ${ }^{8}$ S. Wertz, ${ }^{8}$ N. Beliy, ${ }^{9}$ G. H. Hammad, ${ }^{9}$ W. L. Aldá Júnior, ${ }^{10}$ F. L. Alves, ${ }^{10}$ G. A. Alves, ${ }^{10}$ L. Brito, ${ }^{10}$ M. Correa Martins Junior, ${ }^{10}$ M. Hamer, ${ }^{10}$ C. Hensel, ${ }^{10}$ A. Moraes,${ }^{10}$ M. E. Pol, ${ }^{10}$ P. Rebello Teles, ${ }^{10}$ E. Belchior Batista Das Chagas, ${ }^{11}$ W. Carvalho, ${ }^{11}$ J. Chinellato, ${ }^{11, e}$

A. Custódio, ${ }^{11}$ E. M. Da Costa, ${ }^{11}$ D. De Jesus Damiao, ${ }^{11}$ C. De Oliveira Martins, ${ }^{11}$ S. Fonseca De Souza, ${ }^{11}$

L. M. Huertas Guativa, ${ }^{11}$ H. Malbouisson, ${ }^{11}$ D. Matos Figueiredo, ${ }^{11}$ C. Mora Herrera, ${ }^{11}$ L. Mundim, ${ }^{11}$ H. Nogima,${ }^{11}$

W. L. Prado Da Silva, ${ }^{11}$ A. Santoro, ${ }^{11}$ A. Sznajder, ${ }^{11}$ E. J. Tonelli Manganote, ${ }^{11, e}$ A. Vilela Pereira, ${ }^{11}$ S. Ahuja, ${ }^{12 a}$

C. A. Bernardes, ${ }^{12 b}$ A. De Souza Santos, ${ }^{12 b}$ S. Dogra, ${ }^{12 a}$ T. R. Fernandez Perez Tomei, ${ }^{12 a}$ E. M. Gregores, ${ }^{12 b}$

P. G. Mercadante, ${ }^{12 b}$ C. S. Moon, ${ }^{12 a, f}$ S. F. Novaes, ${ }^{12 a}$ Sandra S. Padula, ${ }^{12 a}$ D. Romero Abad, ${ }^{12 b}$ J. C. Ruiz Vargas, ${ }^{12 a}$ A. Aleksandrov, ${ }^{13}$ R. Hadjiiska, ${ }^{13}$ P. Iaydjiev, ${ }^{13}$ M. Rodozov, ${ }^{13}$ S. Stoykova, ${ }^{13}$ G. Sultanov, ${ }^{13}$ M. Vutova, ${ }^{13}$ A. Dimitrov, ${ }^{14}$ I. Glushkov, ${ }^{14}$ L. Litov, ${ }^{14}$ B. Pavlov, ${ }^{14}$ P. Petkov, ${ }^{14}$ W. Fang, ${ }^{15, g}$ M. Ahmad, ${ }^{16}$ J. G. Bian, ${ }^{16}$ G. M. Chen, ${ }^{16}$ H. S. Chen, ${ }^{16}$ M. Chen, ${ }^{16}$ T. Cheng, ${ }^{16}$ R. Du, ${ }^{16}$ C. H. Jiang, ${ }^{16}$ D. Leggat, ${ }^{16}$ R. Plestina,,${ }^{16}$ F. Romeo, ${ }^{16}$ S. M. Shaheen, ${ }^{16}$ A. Spiezia, ${ }^{16}$ J. Tao, ${ }^{16}$ C. Wang, ${ }^{16}$ Z. Wang, ${ }^{16}$ H. Zhang, ${ }^{16}$ C. Asawatangtrakuldee, ${ }^{17}$ Y. Ban,${ }^{17}$ Q. Li,${ }^{17}$ S. Liu, ${ }^{17}$ Y. Mao, ${ }^{17}$ S. J. Qian, ${ }^{17}$ D. Wang, ${ }^{17}$ Z. Xu, ${ }^{17}$ C. Avila, ${ }^{18}$ A. Cabrera, ${ }^{18}$ L. F. Chaparro Sierra, ${ }^{18}$ C. Florez, ${ }^{18}$ J. P. Gomez, ${ }^{18}$ B. Gomez Moreno, ${ }^{18}$ J. C. Sanabria, ${ }^{18}$ N. Godinovic, ${ }^{19}$ D. Lelas, ${ }^{19}$ I. Puljak, ${ }^{19}$ P. M. Ribeiro Cipriano, ${ }^{19}$ Z. Antunovic, ${ }^{20}$ M. Kovac, ${ }^{20}$ V. Brigljevic, ${ }^{21}$ D. Ferencek,${ }^{21}$ K. Kadija ${ }^{21}$ J. Luetic ${ }^{21}$ S. Micanovic, ${ }^{21}$ L. Sudic, ${ }^{21}$ A. Attikis, ${ }^{22}$ G. Mavromanolakis ${ }^{22}$ J. Mousa, ${ }^{22}$ C. Nicolaou, ${ }^{22}$ F. Ptochos, ${ }^{22}$ P. A. Razis, ${ }^{22}$ H. Rykaczewski, ${ }^{22}$ M. Finger, ${ }^{23, i}$ M. Finger Jr., ${ }^{23, i}$ E. Carrera Jarrin, ${ }^{24}$ A. Awad, ${ }^{25}$ S. Elgammal, ${ }^{25, j}$ A. Mohamed ${ }^{25, k}$ E. Salama, ${ }^{25, j, 1}$ B. Calpas, ${ }^{26}$ M. Kadastik, ${ }^{26}$ M. Murumaa, ${ }^{26}$ L. Perrini ${ }^{26}$ M. Raidal, ${ }^{26}$ A. Tiko, ${ }^{26}$ C. Veelken, ${ }^{26}$ P. Eerola, ${ }^{27}$ J. Pekkanen, ${ }^{27}$ M. Voutilainen, ${ }^{27}$ J. Härkönen, ${ }^{28}$ V. Karimäki, ${ }^{28}$ R. Kinnunen, ${ }^{28}$ T. Lampén, ${ }^{28}$ K. Lassila-Perini, ${ }^{28}$ S. Lehti, ${ }^{28}$ T. Lindén,${ }^{28}$ P. Luukka, ${ }^{28}$ T. Peltola ${ }^{28}$ J. Tuominiemi, ${ }^{28}$ E. Tuovinen,${ }^{28}$ L. Wendland, ${ }^{28}$ J. Talvitie, ${ }^{29}$ T. Tuuva, ${ }^{29}$ M. Besancon, ${ }^{30}$ F. Couderc, ${ }^{30}$ M. Dejardin, ${ }^{30}$ D. Denegri, ${ }^{30}$ B. Fabbro, ${ }^{30}$ J. L. Faure,${ }^{30}$ C. Favaro, ${ }^{30}$ F. Ferri, ${ }^{30}$ S. Ganjour, ${ }^{30}$ A. Givernaud, ${ }^{30}$ P. Gras, ${ }^{30}$ G. Hamel de Monchenault,${ }^{30}$ P. Jarry, ${ }^{30}$ E. Locci, ${ }^{30}$ M. Machet, ${ }^{30}$ J. Malcles, ${ }^{30}$ J. Rander,${ }^{30}$ A. Rosowsky, ${ }^{30}$ M. Titov, ${ }^{30}$ A. Zghiche, ${ }^{30}$ A. Abdulsalam, ${ }^{31}$ I. Antropov, ${ }^{31}$ S. Baffioni, ${ }^{31}$ F. Beaudette, ${ }^{31}$ P. Busson, ${ }^{31}$ L. Cadamuro, ${ }^{31}$ E. Chapon, ${ }^{31}$ C. Charlot,${ }^{31}$ O. Davignon, ${ }^{31}$ L. Dobrzynski, ${ }^{31}$ R. Granier de Cassagnac, ${ }^{31}$ M. Jo,${ }^{31}$ S. Lisniak, ${ }^{31}$ P. Miné, ${ }^{31}$ I. N. Naranjo, ${ }^{31}$ M. Nguyen, ${ }^{31}$ C. Ochando,${ }^{31}$ G. Ortona, ${ }^{31}$ P. Paganini, ${ }^{31}$ P. Pigard, ${ }^{31}$ S. Regnard, ${ }^{31}$ R. Salerno, ${ }^{31}$ Y. Sirois,${ }^{31}$ T. Strebler, ${ }^{31}$ Y. Yilmaz, ${ }^{31}$ A. Zabi, ${ }^{31}$ 
J.-L. Agram, ${ }^{32, \mathrm{~m}}$ J. Andrea, ${ }^{32}$ A. Aubin, ${ }^{32}$ D. Bloch, ${ }^{32}$ J.-M. Brom, ${ }^{32}$ M. Buttignol, ${ }^{32}$ E. C. Chabert, ${ }^{32}$ N. Chanon, ${ }^{32}$ C. Collard, ${ }^{32}$ E. Conte, ${ }^{32, \mathrm{~m}}$ X. Coubez, ${ }^{32}$ J.-C. Fontaine, ${ }^{32, \mathrm{~m}}$ D. Gelé, ${ }^{32}$ U. Goerlach, ${ }^{32}$ C. Goetzmann, ${ }^{32}$ A.-C. Le Bihan, ${ }^{32}$ J. A. Merlin, ${ }^{32, \mathrm{n}}$ K. Skovpen, ${ }^{32}$ P. Van Hove, ${ }^{32}$ S. Gadrat,${ }^{33}$ S. Beauceron,${ }^{34}$ C. Bernet, ${ }^{34}$ G. Boudoul, ${ }^{34}$ E. Bouvier, ${ }^{34}$ C. A. Carrillo Montoya,${ }^{34}$ R. Chierici,${ }^{34}$ D. Contardo,${ }^{34}$ B. Courbon, ${ }^{34}$ P. Depasse, ${ }^{34}$ H. El Mamouni, ${ }^{34}$ J. Fan, ${ }^{34}$ J. Fay, ${ }^{34}$ S. Gascon, ${ }^{34}$ M. Gouzevitch, ${ }^{34}$ B. Ille, ${ }^{34}$ F. Lagarde, ${ }^{34}$ I. B. Laktineh,${ }^{34}$ M. Lethuillier, ${ }^{34}$ L. Mirabito, ${ }^{34}$ A. L. Pequegnot,${ }^{34}$ S. Perries, ${ }^{34}$ A. Popov, ${ }^{34,0}$ J. D. Ruiz Alvarez,${ }^{34}$ D. Sabes, ${ }^{34}$ V. Sordini,${ }^{34}$ M. Vander Donckt, ${ }^{34}$ P. Verdier, ${ }^{34}$ S. Viret,${ }^{34}$ T. Toriashvili, ${ }^{35, p}$ Z. Tsamalaidze, ${ }^{36, i}$ C. Autermann, ${ }^{37}$ S. Beranek, ${ }^{37}$ L. Feld, ${ }^{37}$ A. Heister,${ }^{37}$ M. K. Kiesel, ${ }^{37}$ K. Klein, ${ }^{37}$ M. Lipinski, ${ }^{37}$ A. Ostapchuk, ${ }^{37}$ M. Preuten, ${ }^{37}$ F. Raupach, ${ }^{37}$ S. Schael, ${ }^{37}$ C. Schomakers, ${ }^{37}$ J. F. Schulte, ${ }^{37}$ J. Schulz ${ }^{37}$ T. Verlage, ${ }^{37}$ H. Weber, ${ }^{37}$ V. Zhukov, ${ }^{37,0}$ M. Ata, ${ }^{38}$ M. Brodski, ${ }^{38}$ E. Dietz-Laursonn, ${ }^{38}$ D. Duchardt,${ }^{38}$ M. Endres, ${ }^{38}$ M. Erdmann, ${ }^{38}$ S. Erdweg, ${ }^{38}$ T. Esch, ${ }^{38}$ R. Fischer, ${ }^{38}$ A. Güth, ${ }^{38}$ T. Hebbeker, ${ }^{38}$ C. Heidemann, ${ }^{38}$ K. Hoepfner, ${ }^{38}$ S. Knutzen, ${ }^{38}$ M. Merschmeyer, ${ }^{38}$ A. Meyer, ${ }^{38}$ P. Millet, ${ }^{38}$ S. Mukherjee, ${ }^{38}$ M. Olschewski, ${ }^{38}$ K. Padeken, ${ }^{38}$ P. Papacz, ${ }^{38}$

T. Pook ${ }^{38}$ M. Radziej, ${ }^{38}$ H. Reithler, ${ }^{38}$ M. Rieger, ${ }^{38}$ F. Scheuch ${ }^{38}$ L. Sonnenschein, ${ }^{38}$ D. Teyssier, ${ }^{38}$ S. Thüer, ${ }^{38}$ V. Cherepanov ${ }^{39}$ Y. Erdogan, ${ }^{39}$ G. Flügge, ${ }^{39}$ H. Geenen, ${ }^{39}$ M. Geisler,${ }^{39}$ F. Hoehle,${ }^{39}$ B. Kargoll, ${ }^{39}$ T. Kress, ${ }^{39}$ A. Künsken, ${ }^{39}$ J. Lingemann, ${ }^{39}$ A. Nehrkorn, ${ }^{39}$ A. Nowack, ${ }^{39}$ I. M. Nugent, ${ }^{39}$ C. Pistone, ${ }^{39}$ O. Pooth, ${ }^{39}$ A. Stahl,,${ }^{39, n}$ M. Aldaya Martin, ${ }^{40}$ I. Asin, ${ }^{40}$ K. Beernaert, ${ }^{40}$ O. Behnke, ${ }^{40}$ U. Behrens, ${ }^{40}$ K. Borras, ${ }^{40, q}$ A. Campbell,${ }^{40}$ P. Connor,${ }^{40}$ C. Contreras-Campana,${ }^{40}$ F. Costanza, ${ }^{40}$ C. Diez Pardos, ${ }^{40}$ G. Dolinska, ${ }^{40}$ S. Dooling, ${ }^{40}$ G. Eckerlin, ${ }^{40}$ D. Eckstein, ${ }^{40}$ T. Eichhorn, ${ }^{40}$ E. Gallo, ${ }^{40, r}$ J. Garay Garcia, ${ }^{40}$ A. Geiser, ${ }^{40}$ A. Gizhko, ${ }^{40}$ J. M. Grados Luyando, ${ }^{40}$ P. Gunnellini, ${ }^{40}$ A. Harb,${ }^{40}$ J. Hauk,${ }^{40}$ M. Hempel, ${ }^{40, s}$ H. Jung, ${ }^{40}$ A. Kalogeropoulos, ${ }^{40}$ O. Karacheban, ${ }^{40, s}$ M. Kasemann, ${ }^{40}$ J. Kieseler, ${ }^{40}$ C. Kleinwort, ${ }^{40}$ I. Korol, ${ }^{40}$ W. Lange, ${ }^{40}$ A. Lelek, ${ }^{40}$ J. Leonard,${ }^{40}$ K. Lipka, ${ }^{40}$ A. Lobanov, ${ }^{40}$ W. Lohmann, ${ }^{40, s}$ R. Mankel, ${ }^{40}$ I.-A. Melzer-Pellmann, ${ }^{40}$ A. B. Meyer, ${ }^{40}$ G. Mittag, ${ }^{40}$ J. Mnich,${ }^{40}$ A. Mussgiller, ${ }^{40}$ E. Ntomari, ${ }^{40}$ D. Pitzl, ${ }^{40}$ R. Placakyte, ${ }^{40}$ A. Raspereza, ${ }^{40}$ B. Roland ${ }^{40}$ M. Ö. Sahin, ${ }^{40}$ P. Saxena, ${ }^{40}$ T. Schoerner-Sadenius, ${ }^{40}$ C. Seitz, ${ }^{40}$ S. Spannagel,${ }^{40}$ N. Stefaniuk, ${ }^{40}$ K. D. Trippkewitz, ${ }^{40}$ G. P. Van Onsem, ${ }^{40}$ R. Walsh ${ }^{40}$ C. Wissing, ${ }^{40}$ V. Blobel,${ }^{41}$ M. Centis Vignali, ${ }^{41}$ A. R. Draeger, ${ }^{41}$ T. Dreyer, ${ }^{41}$ J. Erfle, ${ }^{41}$ E. Garutti, ${ }^{41}$ K. Goebel, ${ }^{41}$ D. Gonzalez, ${ }^{41}$ M. Görner, ${ }^{41}$ J. Haller ${ }^{41}$ M. Hoffmann, ${ }^{41}$ R. S. Höing, ${ }^{41}$ A. Junkes, ${ }^{41}$ R. Klanner, ${ }^{41}$ R. Kogler, ${ }^{41}$ N. Kovalchuk, ${ }^{41}$ T. Lapsien, ${ }^{41}$ T. Lenz, ${ }^{41}$ I. Marchesini ${ }^{41}$ D. Marconi, ${ }^{41}$ M. Meyer, ${ }^{41}$ M. Niedziela, ${ }^{41}$ D. Nowatschin, ${ }^{41}$ J. Ott, ${ }^{41}$ F. Pantaleo, ${ }^{41, n}$ T. Peiffer, ${ }^{41}$ A. Perieanu, ${ }^{41}$ N. Pietsch, ${ }^{41}$ J. Poehlsen, ${ }^{41}$ C. Sander, ${ }^{41}$ C. Scharf,${ }^{41}$ P. Schleper, ${ }^{41}$ E. Schlieckau, ${ }^{41}$ A. Schmidt, ${ }^{41}$ S. Schumann, ${ }^{41}$ J. Schwandt,${ }^{41}$ H. Stadie, ${ }^{41}$ G. Steinbrück, ${ }^{41}$ F. M. Stober, ${ }^{41}$ H. Tholen, ${ }^{41}$ D. Troendle, ${ }^{41}$ E. Usai ${ }^{41}$ L. Vanelderen, ${ }^{41}$ A. Vanhoefer, ${ }^{41}$ B. Vormwald, ${ }^{41}$ C. Barth, ${ }^{42}$ C. Baus, ${ }^{42}$ J. Berger, ${ }^{42}$ C. Böser ${ }^{42}$ E. Butz, ${ }^{42}$ T. Chwalek,${ }^{42}$ F. Colombo, ${ }^{42}$ W. De Boer, ${ }^{42}$ A. Descroix ${ }^{42}$ A. Dierlamm, ${ }^{42}$ S. Fink,${ }^{42}$ F. Frensch,${ }^{42}$ R. Friese, ${ }^{42}$ M. Giffels, ${ }^{42}$ A. Gilbert, ${ }^{42}$ D. Haitz, ${ }^{42}$ F. Hartmann, ${ }^{42, n}$ S. M. Heindl, ${ }^{42}$ U. Husemann, ${ }^{42}$ I. Katkov, ${ }^{42, o}$ A. Kornmayer, ${ }^{42, n}$ P. Lobelle Pardo, ${ }^{42}$ B. Maier, ${ }^{42}$ H. Mildner, ${ }^{42}$ M. U. Mozer ${ }^{42}$ T. Müller, ${ }^{42}$ Th. Müller, ${ }^{42}$ M. Plagge, ${ }^{42}$ G. Quast, ${ }^{42}$ K. Rabbertz, ${ }^{42}$ S. Röcker, ${ }^{42}$ F. Roscher, ${ }^{42}$ M. Schröder, ${ }^{42}$ G. Sieber, ${ }^{42}$ H. J. Simonis, ${ }^{42}$ R. Ulrich, ${ }^{42}$ J. Wagner-Kuhr, ${ }^{42}$ S. Wayand, ${ }^{42}$ M. Weber, ${ }^{42}$ T. Weiler, ${ }^{42}$ S. Williamson, ${ }^{42}$ C. Wöhrmann, ${ }^{42}$ R. Wolf,${ }^{42}$ G. Anagnostou, ${ }^{43}$ G. Daskalakis, ${ }^{43}$ T. Geralis, ${ }^{43}$ V. A. Giakoumopoulou, ${ }^{43}$ A. Kyriakis, ${ }^{43}$ D. Loukas, ${ }^{43}$ A. Psallidas, ${ }^{43}$ I. Topsis-Giotis, ${ }^{43}$ A. Agapitos, ${ }^{44}$ S. Kesisoglou, ${ }^{44}$ A. Panagiotou, ${ }^{44}$ N. Saoulidou, ${ }^{44}$ E. Tziaferi, ${ }^{44}$ I. Evangelou, ${ }^{45}$ G. Flouris, ${ }^{45}$ C. Foudas, ${ }^{45}$ P. Kokkas, ${ }^{45}$ N. Loukas, ${ }^{45}$ N. Manthos,${ }^{45}$ I. Papadopoulos,${ }^{45}$ E. Paradas,${ }^{45}$ J. Strologas ${ }^{45}$ N. Filipovic,${ }^{46}$ G. Bencze, ${ }^{47}$ C. Hajdu, ${ }^{47}$ P. Hidas, ${ }^{47}$ D. Horvath, ${ }^{47, t}$ F. Sikler, ${ }^{47}$ V. Veszpremi, ${ }^{47}$ G. Vesztergombi, ${ }^{4, u}$ A. J. Zsigmond, ${ }^{47}$ N. Beni, ${ }^{48}$ S. Czellar, ${ }^{48}$ J. Karancsi, ${ }^{48, v}$ J. Molnar, ${ }^{48}$ Z. Szillasi, ${ }^{48}$ M. Bartók, ${ }^{49, u}$ A. Makovec, ${ }^{49}$ P. Raics, ${ }^{49}$ Z. L. Trocsanyi, ${ }^{49}$ B. Ujvari, ${ }^{49}$ S. Choudhury, ${ }^{50, w}$ P. Mal, ${ }^{50}$ K. Mandal,${ }^{50}$ A. Nayak, ${ }^{50}$ D. K. Sahoo,${ }^{50}$ N. Sahoo, ${ }^{50}$ S. K. Swain,${ }^{50}$ S. Bansal, ${ }^{51}$ S. B. Beri ${ }^{51}$ V. Bhatnagar, ${ }^{51}$ R. Chawla, ${ }^{51}$ R. Gupta,${ }^{51}$ U. Bhawandeep, ${ }^{51}$ A. K. Kalsi, ${ }^{51}$ A. Kaur, ${ }^{51}$ M. Kaur, ${ }^{51}$ R. Kumar, ${ }^{51}$ A. Mehta, ${ }^{51}$ M. Mittal, ${ }^{51}$ J. B. Singh, ${ }^{51}$ G. Walia, ${ }^{51}$ Ashok Kumar, ${ }^{52}$ A. Bhardwaj, ${ }^{52}$ B. C. Choudhary, ${ }^{52}$ R. B. Garg, ${ }^{52}$ S. Keshri, ${ }^{52}$ A. Kumar, ${ }^{52}$ S. Malhotra, ${ }^{52}$ M. Naimuddin, ${ }^{52}$ N. Nishu, ${ }^{52}$ K. Ranjan, ${ }^{52}$ R. Sharma ${ }^{52}$ V. Sharma, ${ }^{52}$ R. Bhattacharya, ${ }^{53}$ S. Bhattacharya, ${ }^{53}$ K. Chatterjee, ${ }^{53}$ S. Dey, ${ }^{53}$ S. Dutta ${ }^{53}$ S. Ghosh, ${ }^{53}$ N. Majumdar, ${ }^{53}$ A. Modak, ${ }^{53}$ K. Mondal ${ }^{53}$ S. Mukhopadhyay, ${ }^{53}$ S. Nandan,${ }^{53}$ A. Purohit, ${ }^{53}$ A. Roy, ${ }^{53}$ D. Roy,${ }^{53}$ S. Roy Chowdhury,${ }^{53}$ S. Sarkar, ${ }^{53}$ M. Sharan, ${ }^{53}$ R. Chudasama, ${ }^{54}$ D. Dutta ${ }^{54}$ V. Jha, ${ }^{54}$ V. Kumar, ${ }^{54}$ A. K. Mohanty, ${ }^{54}$ L. M. Pant,${ }^{54}$ P. Shukla,${ }^{54}$ A. Topkar,${ }^{54}$ T. Aziz, ${ }^{55}$ S. Banerjee, ${ }^{55}$ S. Bhowmik, ${ }^{55, \mathrm{x}}$ R. M. Chatterjee, ${ }^{55}$ R. K. Dewanjee, ${ }^{55}$ S. Dugad, ${ }^{55}$ S. Ganguly, ${ }^{55}$ S. Ghosh, ${ }^{55}$ M. Guchait, ${ }^{55}$ A. Gurtu, ${ }^{55, y}$ Sa. Jain, ${ }^{55}$ G. Kole, ${ }^{55}$ S. Kumar, ${ }^{55}$ B. Mahakud ${ }^{55}$ M. Maity, ${ }^{55, x}$ G. Majumder, ${ }^{55}$ K. Mazumdar, ${ }^{55}$ S. Mitra, ${ }^{55}$ G. B. Mohanty, ${ }^{55}$ B. Parida, ${ }^{55}$ T. Sarkar, ${ }^{55, x}$ N. Sur, ${ }^{55}$ B. Sutar, ${ }^{55}$ N. Wickramage, ${ }^{55, z}$ S. Chauhan, ${ }^{56}$ S. Dube, ${ }^{56}$ A. Kapoor, ${ }^{56}$ K. Kothekar, ${ }^{56}$ A. Rane, ${ }^{56}$ S. Sharma ${ }^{56}$ H. Bakhshiansohi, ${ }^{57}$ H. Behnamian, ${ }^{57}$ S. M. Etesami, ${ }^{57, \text { aa }}$ A. Fahim, ${ }^{57, b b}$ M. Khakzad, ${ }^{57}$ M. Mohammadi Najafabadi, ${ }^{57}$ M. Naseri, ${ }^{57}$ S. Paktinat Mehdiabadi, ${ }^{57}$ 
F. Rezaei Hosseinabadi, ${ }^{57}$ B. Safarzadeh, ${ }^{57, c c}$ M. Zeinali, ${ }^{57}$ M. Felcini, ${ }^{58}$ M. Grunewald, ${ }^{58}$ M. Abbrescia, ${ }^{59 a, 59 b}$

C. Calabria,${ }^{59 a, 59 b}$ C. Caputo, ${ }^{59 a, 59 b}$ A. Colaleo ${ }^{59 a}$ D. Creanza, ${ }^{59 a, 59 c}$ L. Cristella,${ }^{59 a, 59 b}$ N. De Filippis,${ }^{59,59 c}$

M. De Palma ${ }^{59 a, 59 b}$ L. Fiore, ${ }^{59 a}$ G. Iaselli, ${ }^{59 a, 59 c}$ G. Maggi, ${ }^{59 a, 59 c}$ M. Maggi, ${ }^{59 a}$ G. Miniello,,${ }^{59 a, 59 b}$ S. My, ${ }^{59 a, 59 b}$ S. Nuzzo, ${ }^{59 a, 59 b}$ A. Pompili, ${ }^{59 a, 59 b}$ G. Pugliese,${ }^{59 a, 59 c}$ R. Radogna,${ }^{59 a, 59 b}$ A. Ranieri, ${ }^{59 a}$ G. Selvaggi, ${ }^{59,59 b}$ L. Silvestris,${ }^{59 a, n}$ R. Venditti, ${ }^{59 a, 59 b}$ G. Abbiendi, ${ }^{60 \mathrm{a}}$ C. Battilana, ${ }^{60 \mathrm{a}}$ D. Bonacorsi, ${ }^{60 \mathrm{a}, 60 \mathrm{~b}}$ S. Braibant-Giacomelli, ${ }^{60 \mathrm{a}, 60 \mathrm{~b}}$ L. Brigliadori, ${ }^{60 \mathrm{a}, 60 \mathrm{~b}}$ R. Campanini, ${ }^{60 \mathrm{a}, 60 \mathrm{~b}}$

P. Capiluppi ${ }^{60 \mathrm{a}, 60 \mathrm{~b}}$ A. Castro, ${ }^{60 \mathrm{a}, 60 \mathrm{~b}}$ F. R. Cavallo, ${ }^{60 \mathrm{a}}$ S. S. Chhibra, ${ }^{60,60 \mathrm{~b}}$ G. Codispoti, ${ }^{60 \mathrm{a}, 60 \mathrm{~b}}$ M. Cuffiani, ${ }^{60 \mathrm{a}, 60 \mathrm{~b}}$ G. M. Dallavalle, ${ }^{60 \mathrm{a}}$ F. Fabbri, ${ }^{60 \mathrm{a}}$ A. Fanfani, ${ }^{60 \mathrm{a}, 60 \mathrm{~b}}$ D. Fasanella, ${ }^{60 \mathrm{a}, 60 \mathrm{~b}}$ P. Giacomelli, ${ }^{60 \mathrm{a}}$ C. Grandi, ${ }^{60 \mathrm{a}}$ L. Guiducci, ${ }^{60 \mathrm{a}, 60 \mathrm{~b}}$ S. Marcellini, ${ }^{60 a}$ G. Masetti, ${ }^{60 a}$ A. Montanari, ${ }^{60 a}$ F. L. Navarria, ${ }^{60 a, 60 b}$ A. Perrotta, ${ }^{60 a}$ A. M. Rossi, ${ }^{60 a, 60 b}$ T. Rovelli, ${ }^{60 a, 60 b}$ G. P. Siroli, ${ }^{60,60 b}$ N. Tosi, ${ }^{6 a, 60 b, n}$ G. Cappello, ${ }^{61 b}$ M. Chiorboli, ${ }^{61 a, 61 b}$ S. Costa, ${ }^{61 a, 61 b}$ A. Di Mattia, ${ }^{61 a}$ F. Giordano, ${ }^{61 a, 61 b}$ R. Potenza, ${ }^{61 a, 61 b}$ A. Tricomi, ${ }^{61 a, 61 b}$ C. Tuve,${ }^{61 a, 61 b}$ G. Barbagli, ${ }^{62 a}$ V. Ciulli, ${ }^{62 a, 62 b}$ C. Civinini, ${ }^{62 a}$ R. D'Alessandro, ${ }^{62 a, 62 b}$ E. Focardi, ${ }^{62 a, 62 b}$ V. Gori, ${ }^{62 a, 62 b}$ P. Lenzi, ${ }^{62 a, 62 b}$ M. Meschini, ${ }^{62 a}$ S. Paoletti, ${ }^{62 a}$ G. Sguazzoni, ${ }^{62 a}$ L. Viliani, ${ }^{62 a, 62 b, n}$

L. Benussi ${ }^{63}$ S. Bianco, ${ }^{63}$ F. Fabbri, ${ }^{63}$ D. Piccolo, ${ }^{63}$ F. Primavera ${ }^{63, n}$ V. Calvelli, ${ }^{64 a, 64 b}$ F. Ferro,${ }^{64 a}$ M. Lo Vetere, ${ }^{64 a, 64 b}$ M. R. Monge,${ }^{64 a, 64 b}$ E. Robutti, ${ }^{64 a}$ S. Tosi, ${ }^{64 a, 64 b}$ L. Brianza, ${ }^{65 a}$ M. E. Dinardo, ${ }^{65 a, 65 b}$ S. Fiorendi, ${ }^{65 a, 65 b}$ S. Gennai, ${ }^{65 a}$ A. Ghezzi, ${ }^{65 a, 65 b}$ P. Govoni, ${ }^{65 a, 65 b}$ S. Malvezzi, ${ }^{65 a}$ R. A. Manzoni, ${ }^{65 a, 65 b, n}$ B. Marzocchi, ${ }^{65 a, 65 b}$ D. Menasce, ${ }^{65 a}$ L. Moroni, ${ }^{65 a}$ M. Paganoni, ${ }^{65 a, 65 b}$ D. Pedrini, ${ }^{65 a}$ S. Pigazzini, ${ }^{65 a}$ S. Ragazzi, ${ }^{65 a, 65 b}$ N. Redaelli, ${ }^{65 a}$ T. Tabarelli de Fatis, ${ }^{65 a, 65 b}$ S. Buontempo, ${ }^{66 \mathrm{a}}$ N. Cavallo, ${ }^{66 a, 66 \mathrm{c}}$ S. Di Guida ${ }^{66 \mathrm{a}, 66 \mathrm{~d}, \mathrm{n}}$ M. Esposito, ${ }^{66 \mathrm{a}, 66 \mathrm{~b}}$ F. Fabozzi, ${ }^{66 a, 66 \mathrm{c}}$ A. O. M. Iorio, ${ }^{66 \mathrm{a}, 66 \mathrm{~b}}$ G. Lanza, ${ }^{66 \mathrm{a}}$ L. Lista, ${ }^{66 \mathrm{a}}$ S. Meola,${ }^{66 \mathrm{a}, 66 \mathrm{~d}, \mathrm{n}}$ M. Merola, ${ }^{66 \mathrm{a}}$ P. Paolucci, ${ }^{66 a, n}$ C. Sciacca, ${ }^{66 a, 66 \mathrm{~b}}$ F. Thyssen, ${ }^{66 \mathrm{a}}$ P. Azzi, ${ }^{67 a, n}$ N. Bacchetta, ${ }^{67 \mathrm{a}}$ M. Bellato, ${ }^{67 \mathrm{a}}$ L. Benato, ${ }^{67,67 \mathrm{~b}}$ D. Bisello, ${ }^{67 \mathrm{a}, 67 \mathrm{~b}}$ A. Boletti, ${ }^{67 a, 67 b}$ A. Branca, ${ }^{67 a, 67 b}$ R. Carlin, ${ }^{67,67 b}$ A. Carvalho Antunes De Oliveira, ${ }^{67,67 b}$ P. Checchia, ${ }^{67 a}$ M. Dall'Osso, ${ }^{67 a, 67 b}$ P. De Castro Manzano, ${ }^{67 a}$ T. Dorigo, ${ }^{67 a}$

U. Dosselli, ${ }^{67 a}$ F. Gasparini, ${ }^{67 a, 67 b}$ U. Gasparini, ${ }^{67 a, 67 b}$ A. Gozzelino, ${ }^{67 a}$ S. Lacaprara, ${ }^{67 a}$ M. Margoni, ${ }^{67 a, 67 b}$

A. T. Meneguzzo, ${ }^{67 a, 67 b}$ J. Pazzini, ${ }^{67 a, 67 b, n}$ N. Pozzobon, ${ }^{67 a, 67 b}$ P. Ronchese,${ }^{67,67 b}$ F. Simonetto,${ }^{67 a, 67 b}$ E. Torassa, ${ }^{67 a}$ M. Tosi, ${ }^{67 a, 67 b}$ M. Zanetti, ${ }^{67 a}$ P. Zotto, ${ }^{67 a, 67 b}$ A. Zucchetta, ${ }^{67 a, 67 b}$ G. Zumerle, ${ }^{67 a, 67 b}$ A. Braghieri, ${ }^{68 \mathrm{a}}$ A. Magnani, ${ }^{68 a, 68 \mathrm{~b}}$ P. Montagna, ${ }^{68 a, 68 b}$ S. P. Ratti ${ }^{68 a, 68 b}$ V. Re ${ }^{68 a}$ C. Riccardi ${ }^{68 a, 68 b}$ P. Salvini ${ }^{68 a}$ I. Vai, ${ }^{68 a, 68 b}$ P. Vitulo, ${ }^{68 a, 68 b}$

L. Alunni Solestizi, ${ }^{69 a, 69 b}$ G. M. Bilei, ${ }^{69 a}$ D. Ciangottini,,${ }^{6 a, 69 b}$ L. Fanò,${ }^{69 a, 69 b}$ P. Lariccia, ${ }^{69 a, 69 b}$ R. Leonardi, ${ }^{69 a, 69 b}$ G. Mantovani, ${ }^{69 a, 69 b}$ M. Menichelli, ${ }^{69 a}$ A. Saha, ${ }^{69 a}$ A. Santocchia, ${ }^{69 a, 69 b}$ K. Androsov, ${ }^{70 a, d d}$ P. Azzurri, ${ }^{70 a, n}$ G. Bagliesi, ${ }^{70 a}$ J. Bernardini, ${ }^{70 a}$ T. Boccali, ${ }^{70 a}$ R. Castaldi, ${ }^{70 a}$ M. A. Ciocci ${ }^{70 a, d d}$ R. Dell'Orso, ${ }^{70 a}$ S. Donato, ${ }^{70,70 \mathrm{c}}$ G. Fedi, ${ }^{70 a}$ A. Giassi, ${ }^{70 a}$ M. T. Grippo, ${ }^{70 a, d d}$ F. Ligabue,${ }^{70 a, 70 c}$ T. Lomtadze, ${ }^{70 a}$ L. Martini, ${ }^{70 a, 70 b}$ A. Messineo,${ }^{70 a, 70 b}$ F. Palla, ${ }^{70 a}$ A. Rizzi, ${ }^{70 a, 70 b}$ A. Savoy-Navarro, ${ }^{70 a, e e}$ P. Spagnolo, ${ }^{70 a}$ R. Tenchini, ${ }^{70 a}$ G. Tonelli, ${ }^{70 a, 70 b}$ A. Venturi, ${ }^{70 a}$ P. G. Verdini,${ }^{70 a}$ L. Barone, ${ }^{71 a, 71 b}$ F. Cavallari, ${ }^{71 a}$ G. D'imperio, ${ }^{71 a, 71 b, n}$ D. Del Re ${ }^{71 a, 71 b, n}$ M. Diemoz, ${ }^{71 a}$ S. Gelli, ${ }^{71 a, 71 b}$ C. Jorda, ${ }^{71 a}$ E. Longo, ${ }^{71 a, 71 b}$ F. Margaroli, ${ }^{71,71 b}$ P. Meridiani, ${ }^{71 a}$ G. Organtini, ${ }^{71 a, 71 b}$ R. Paramatti, ${ }^{71 a}$ F. Preiato, ${ }^{71 a, 71 b}$ S. Rahatlou, ${ }^{71 a, 71 b}$ C. Rovelli, ${ }^{71 a}$ F. Santanastasio, ${ }^{71 a, 71 b}$ N. Amapane ${ }^{72 a, 72 b}$ R. Arcidiacono,${ }^{72 a, 72 c, n}$ S. Argiro, ${ }^{72 a, 72 b}$ M. Arneodo ${ }^{72 a, 72 c}$ N. Bartosik, ${ }^{72 a}$ R. Bellan, ${ }^{72 a, 72 b}$ C. Biino, ${ }^{72 a}$ N. Cartiglia, ${ }^{72 a}$ M. Costa, ${ }^{72 a, 72 b}$ R. Covarelli, ${ }^{72 a, 72 b}$ A. Degano, ${ }^{72 a, 72 b}$ N. Demaria, ${ }^{72 a}$ L. Finco, ${ }^{72 a, 72 b}$ B. Kiani, ${ }^{72 a, 72 b}$ C. Mariotti, ${ }^{72 a}$ S. Maselli, ${ }^{72 a}$ E. Migliore, ${ }^{72 a, 72 b}$ V. Monaco, ${ }^{72 a, 72 b}$ E. Monteil, ${ }^{72 a, 72 b}$ M. M. Obertino, ${ }^{72 a, 72 b}$ L. Pacher, ${ }^{72 a, 72 b}$ N. Pastrone, ${ }^{72 a}$ M. Pelliccioni ${ }^{72 a}$ G. L. Pinna Angioni, ${ }^{72 a, 72 b}$ F. Ravera, ${ }^{72 a, 72 b}$ A. Romero, ${ }^{72 a, 72 b}$ M. Ruspa,${ }^{72 a, 72 c}$ R. Sacchi,${ }^{72 a, 72 b}$ V. Sola,${ }^{72 a}$ A. Solano, ${ }^{72 a, 72 b}$ A. Staiano, ${ }^{72 a}$ P. Traczyk, ${ }^{72 a, 72 b}$ S. Belforte, ${ }^{73 a}$ V. Candelise, ${ }^{73 a, 73 b}$ M. Casarsa, ${ }^{73 a}$ F. Cossutti, ${ }^{73 a}$ G. Della Ricca, ${ }^{73 a, 73 b}$ C. La Licata, ${ }^{73 a, 73 b}$ A. Schizzi, ${ }^{73 a, 73 b}$ A. Zanetti, ${ }^{73 a}$ S. K. Nam,${ }^{74}$ D. H. Kim,${ }^{75}$ G. N. Kim, ${ }^{75}$ M. S. Kim,${ }^{75}$ D. J. Kong, ${ }^{75}$ S. Lee, ${ }^{75}$ S. W. Lee, ${ }^{75}$ Y. D. Oh,${ }^{75}$ A. Sakharov, ${ }^{75}$ D. C. Son,${ }^{75}$ Y. C. Yang, ${ }^{75}$ J. A. Brochero Cifuentes, ${ }^{76}$ H. Kim, ${ }^{76}$ T. J. Kim,${ }^{76, f f}$ S. Song, ${ }^{77}$ S. Cho, ${ }^{78}$ S. Choi, ${ }^{78}$ Y. Go, ${ }^{78}$ D. Gyun, ${ }^{78}$ B. Hong ${ }^{78}$ Y. Jo, ${ }^{78}$ Y. Kim, ${ }^{78}$ B. Lee, ${ }^{78}$ K. Lee, ${ }^{78}$ K. S. Lee, ${ }^{78}$ S. Lee, ${ }^{78}$ J. Lim, ${ }^{78}$ S. K. Park, ${ }^{78}$ Y. Roh, ${ }^{78}$ H. D. Yoo, ${ }^{79}$ M. Choi ${ }^{80}$ H. Kim, ${ }^{80}$ H. Kim, ${ }^{80}$ J. H. Kim, ${ }^{80}$ J. S. H. Lee, ${ }^{80}$ I. C. Park, ${ }^{80}$ G. Ryu, ${ }^{80}$ M. S. Ryu, ${ }^{80}$ Y. Choi, ${ }^{81}$ J. Goh ${ }^{81}$ D. Kim, ${ }^{81}$ E. Kwon, ${ }^{81}$ J. Lee,${ }^{81}$ I. Yu, ${ }^{81}$ V. Dudenas, ${ }^{82}$ A. Juodagalvis, ${ }^{82}$ J. Vaitkus, ${ }^{82}$ I. Ahmed ${ }^{83}$ Z. A. Ibrahim, ${ }^{83}$ J. R. Komaragiri, ${ }^{83}$ M. A. B. Md Ali, ${ }^{83, g g}$ F. Mohamad Idris, ${ }^{83, \text { hh }}$ W. A. T. Wan Abdullah, ${ }^{83}$ M. N. Yusli, ${ }^{83}$ Z. Zolkapli, ${ }^{83}$ E. Casimiro Linares ${ }^{84}$ H. Castilla-Valdez, ${ }^{84}$ E. De La Cruz-Burelo, ${ }^{84}$ I. Heredia-De La Cruz, ${ }^{84, \text { ii }}$ A. Hernandez-Almada, ${ }^{84}$ R. Lopez-Fernandez ${ }^{84}$ J. Mejia Guisao, ${ }^{84}$ A. Sanchez-Hernandez ${ }^{84}$ S. Carrillo Moreno, ${ }^{85}$ F. Vazquez Valencia, ${ }^{85}$ I. Pedraza, ${ }^{86}$ H. A. Salazar Ibarguen,${ }^{86}$ C. Uribe Estrada, ${ }^{86}$ A. Morelos Pineda,${ }^{87}$ D. Krofcheck, ${ }^{88}$ P. H. Butler, ${ }^{89}$ A. Ahmad, ${ }^{90}$ M. Ahmad, ${ }^{90}$ Q. Hassan, ${ }^{90}$ H. R. Hoorani, ${ }^{90}$ W. A. Khan, ${ }^{90}$ T. Khurshid, ${ }^{90}$ M. Shoaib,${ }^{90}$ M. Waqas, ${ }^{90}$ H. Bialkowska, ${ }^{91}$ M. Bluj,${ }^{91}$ B. Boimska, ${ }^{91}$ T. Frueboes,${ }^{91}$ M. Górski, ${ }^{91}$ M. Kazana,${ }^{91}$ K. Nawrocki, ${ }^{91}$ K. Romanowska-Rybinska, ${ }^{91}$ M. Szleper, ${ }^{91}$ P. Zalewski, ${ }^{91}$ G. Brona,${ }^{92}$ K. Bunkowski, ${ }^{92}$ A. Byszuk, ${ }^{92, j j}$ K. Doroba, ${ }^{92}$ A. Kalinowski, ${ }^{92}$ M. Konecki, ${ }^{92}$ J. Krolikowski, ${ }^{92}$ M. Misiura, ${ }^{92}$ M. Olszewski, ${ }^{92}$ M. Walczak, ${ }^{92}$ P. Bargassa, ${ }^{93}$ C. Beirão Da Cruz E Silva, ${ }^{93}$ 
A. Di Francesco, ${ }^{93}$ P. Faccioli ${ }^{93}$ P. G. Ferreira Parracho, ${ }^{93}$ M. Gallinaro, ${ }^{93}$ J. Hollar ${ }^{93}$ N. Leonardo,${ }^{93}$ L. Lloret Iglesias,${ }^{93}$ M. V. Nemallapudi, ${ }^{93}$ F. Nguyen, ${ }^{93}$ J. Rodrigues Antunes, ${ }^{93}$ J. Seixas, ${ }^{93}$ O. Toldaiev, ${ }^{93}$ D. Vadruccio,${ }^{93}$ J. Varela, ${ }^{93}$ P. Vischia, ${ }^{93}$ S. Afanasiev, ${ }^{94}$ P. Bunin, ${ }^{94}$ I. Golutvin, ${ }^{94}$ A. Kamenev, ${ }^{94}$ V. Karjavin, ${ }^{94}$ V. Korenkov, ${ }^{94}$ A. Lanev, ${ }^{94}$ A. Malakhov, ${ }^{94}$ V. Matveev, ${ }^{94, k k, 11}$ V. V. Mitsyn,${ }^{94}$ P. Moisenz,${ }^{94}$ V. Palichik,${ }^{94}$ V. Perelygin,,${ }^{94}$ M. Savina, ${ }^{94}$ S. Shmatov,${ }^{94}$ N. Skatchkov, ${ }^{94}$ V. Smirnov, ${ }^{94}$ N. Voytishin, ${ }^{94}$ A. Zarubin, ${ }^{94}$ V. Golovtsov, ${ }^{95}$ Y. Ivanov, ${ }^{95}$ V. Kim, ${ }^{95, m m}$ E. Kuznetsova,,${ }^{95, n n}$ P. Levchenko, ${ }^{95}$ V. Murzin, ${ }^{95}$ V. Oreshkin, ${ }^{95}$ I. Smirnov, ${ }^{95}$ V. Sulimov, ${ }^{95}$ L. Uvarov, ${ }^{95}$ S. Vavilov,${ }^{95}$ A. Vorobyev,${ }^{95}$ Yu. Andreev, ${ }^{96}$ A. Dermenev, ${ }^{96}$ S. Gninenko, ${ }^{96}$ N. Golubev, ${ }^{96}$ A. Karneyeu, ${ }^{96}$ M. Kirsanov, ${ }^{96}$ N. Krasnikov, ${ }^{96}$ A. Pashenkov, ${ }^{96}$ D. Tlisov, ${ }^{96}$ A. Toropin, ${ }^{96}$ V. Epshteyn, ${ }^{97}$ V. Gavrilov,${ }^{97}$ N. Lychkovskaya, ${ }^{97}$ V. Popov, ${ }^{97}$ I. Pozdnyakov,${ }^{97}$ G. Safronov, ${ }^{97}$ A. Spiridonov, ${ }^{97}$ M. Toms,${ }^{97}$ E. Vlasov, ${ }^{97}$ A. Zhokin, ${ }^{97}$ M. Chadeeva,${ }^{98}$ R. Chistov, ${ }^{98}$ M. Danilov,${ }^{98}$ O. Markin, ${ }^{98}$ E. Tarkovskii, ${ }^{98}$ V. Andreev, ${ }^{99}$ M. Azarkin,,${ }^{99,11}$ I. Dremin, ${ }^{99,11}$ M. Kirakosyan, ${ }^{99}$ A. Leonidov, ${ }^{99,11}$ G. Mesyats, ${ }^{99}$ S. V. Rusakov, ${ }^{99}$ A. Baskakov, ${ }^{100}$ A. Belyaev, ${ }^{100}$ E. Boos, ${ }^{100}$ V. Bunichev, ${ }^{100}$ M. Dubinin, ${ }^{100,00}$ L. Dudko, ${ }^{100}$ A. Gribushin, ${ }^{100}$ V. Klyukhin, ${ }^{100}$ O. Kodolova, ${ }^{100}$ I. Lokhtin, ${ }^{100}$ I. Miagkov, ${ }^{100}$ S. Obraztsov, ${ }^{100}$ S. Petrushanko, ${ }^{100}$ V. Savrin, ${ }^{100}$

A. Snigirev,${ }^{100}$ I. Azhgirey, ${ }^{101}$ I. Bayshev, ${ }^{101}$ S. Bitioukov, ${ }^{101}$ V. Kachanov, ${ }^{101}$ A. Kalinin, ${ }^{101}$ D. Konstantinov, ${ }^{101}$ V. Krychkine, ${ }^{101}$ V. Petrov ${ }^{101}$ R. Ryutin, ${ }^{101}$ A. Sobol,${ }^{101}$ L. Tourtchanovitch, ${ }^{101}$ S. Troshin,${ }^{101}$ N. Tyurin, ${ }^{101}$ A. Uzunian, ${ }^{101}$ A. Volkov, ${ }^{101}$ P. Adzic, ${ }^{102, p p}$ P. Cirkovic, ${ }^{102}$ D. Devetak, ${ }^{102}$ J. Milosevic, ${ }^{102}$ V. Rekovic, ${ }^{102}$ J. Alcaraz Maestre,${ }^{103}$ E. Calvo, ${ }^{103}$ M. Cerrada, ${ }^{103}$ M. Chamizo Llatas, ${ }^{103}$ N. Colino, ${ }^{103}$ B. De La Cruz, ${ }^{103}$ A. Delgado Peris, ${ }^{103}$ A. Escalante Del Valle, ${ }^{103}$ C. Fernandez Bedoya, ${ }^{103}$ J. P. Fernández Ramos, ${ }^{103}$ J. Flix,${ }^{103}$ M. C. Fouz, ${ }^{103}$ P. Garcia-Abia, ${ }^{103}$ O. Gonzalez Lopez, ${ }^{103}$ S. Goy Lopez, ${ }^{103}$ J. M. Hernandez, ${ }^{103}$ M. I. Josa, ${ }^{103}$ E. Navarro De Martino, ${ }^{103}$ A. Pérez-Calero Yzquierdo, ${ }^{103}$ J. Puerta Pelayo, ${ }^{103}$ A. Quintario Olmeda, ${ }^{103}$ I. Redondo, ${ }^{103}$ L. Romero, ${ }^{103}$ M. S. Soares, ${ }^{103}$ J. F. de Trocóniz, ${ }^{104}$ M. Missiroli, ${ }^{104}$ D. Moran, ${ }^{104}$ J. Cuevas, ${ }^{105}$ J. Fernandez Menendez, ${ }^{105}$ S. Folgueras, ${ }^{105}$ I. Gonzalez Caballero, ${ }^{105}$ E. Palencia Cortezon, ${ }^{105}$ J. M. Vizan Garcia, ${ }^{105}$ I. J. Cabrillo, ${ }^{106}$ A. Calderon, ${ }^{106}$ J. R. Castiñeiras De Saa, ${ }^{106}$ E. Curras, ${ }^{106}$ M. Fernandez, ${ }^{106}$ J. Garcia-Ferrero, ${ }^{106}$ G. Gomez, ${ }^{106}$ A. Lopez Virto, ${ }^{106}$ J. Marco, ${ }^{106}$ R. Marco, ${ }^{106}$ C. Martinez Rivero, ${ }^{106}$

F. Matorras, ${ }^{106}$ J. Piedra Gomez, ${ }^{106}$ T. Rodrigo, ${ }^{106}$ A. Y. Rodríguez-Marrero, ${ }^{106}$ A. Ruiz-Jimeno, ${ }^{106}$ L. Scodellaro, ${ }^{106}$

N. Trevisani, ${ }^{106}$ I. Vila, ${ }^{106}$ R. Vilar Cortabitarte, ${ }^{106}$ D. Abbaneo ${ }^{107}$ E. Auffray,${ }^{107}$ G. Auzinger, ${ }^{107}$ M. Bachtis, ${ }^{107}$ P. Baillon, ${ }^{107}$ A. H. Ball, ${ }^{107}$ D. Barney,${ }^{107}$ A. Benaglia, ${ }^{107}$ L. Benhabib,${ }^{107}$ G. M. Berruti, ${ }^{107}$ P. Bloch,${ }^{107}$ A. Bocci, ${ }^{107}$ A. Bonato, ${ }^{107}$ C. Botta, ${ }^{107}$ H. Breuker ${ }^{107}$ T. Camporesi, ${ }^{107}$ R. Castello, ${ }^{107}$ M. Cepeda,${ }^{107}$ G. Cerminara,${ }^{107}$ M. D' Alfonso, ${ }^{107}$ D. d'Enterria, ${ }^{107}$ A. Dabrowski, ${ }^{107}$ V. Daponte, ${ }^{107}$ A. David,${ }^{107}$ M. De Gruttola,${ }^{107}$ F. De Guio, ${ }^{107}$ A. De Roeck, ${ }^{107}$ E. Di Marco, ${ }^{107, q q}$ M. Dobson, ${ }^{107}$ M. Dordevic, ${ }^{107}$ B. Dorney, ${ }^{107}$ T. du Pree,${ }^{107}$ D. Duggan, ${ }^{107}$ M. Dünser,${ }^{107}$ N. Dupont,${ }^{107}$ A. Elliott-Peisert, ${ }^{107}$ S. Fartoukh, ${ }^{107}$ G. Franzoni, ${ }^{107}$ J. Fulcher ${ }^{107}$ W. Funk,${ }^{107}$ D. Gigi, ${ }^{107}$ K. Gill,,${ }^{107}$ M. Girone, ${ }^{107}$ F. Glege,${ }^{107}$ R. Guida, ${ }^{107}$ S. Gundacker, ${ }^{107}$ M. Guthoff, ${ }^{107}$ J. Hammer, ${ }^{107}$ P. Harris, ${ }^{107}$ J. Hegeman, ${ }^{107}$ V. Innocente,${ }^{107}$ P. Janot, ${ }^{107}$ H. Kirschenmann, ${ }^{107}$ V. Knünz, ${ }^{107}$ M. J. Kortelainen, ${ }^{107}$ K. Kousouris, ${ }^{107}$ P. Lecoq ${ }^{107}$ C. Lourenço, ${ }^{107}$ M. T. Lucchini, ${ }^{107}$ N. Magini, ${ }^{107}$ L. Malgeri, ${ }^{107}$ M. Mannelli, ${ }^{107}$ A. Martelli, ${ }^{107}$ L. Masetti, ${ }^{107}$ F. Meijers, ${ }^{107}$ S. Mersi,${ }^{107}$ E. Meschi,${ }^{107}$ F. Moortgat, ${ }^{107}$ S. Morovic, ${ }^{107}$ M. Mulders,${ }^{107}$ H. Neugebauer, ${ }^{107}$ S. Orfanelli, ${ }^{107, \text {,r }}$ L. Orsini, ${ }^{107}$ L. Pape, ${ }^{107}$ E. Perez,${ }^{107}$ M. Peruzzi, ${ }^{107}$ A. Petrilli, ${ }^{107}$ G. Petrucciani, ${ }^{107}$ A. Pfeiffer, ${ }^{107}$ M. Pierini, ${ }^{107}$ D. Piparo, ${ }^{107}$ A. Racz, ${ }^{107}$ T. Reis, ${ }^{107}$ G. Rolandi, ${ }^{107, s s}$ M. Rovere, ${ }^{107}$ M. Ruan, ${ }^{107}$ H. Sakulin, ${ }^{107}$ J. B. Sauvan, ${ }^{107}$ C. Schäfer, ${ }^{107}$ C. Schwick, ${ }^{107}$ M. Seidel, ${ }^{107}$ A. Sharma, ${ }^{107}$ P. Silva, ${ }^{107}$ M. Simon, ${ }^{107}$ P. Sphicas, ${ }^{107, t t}$ J. Steggemann, ${ }^{107}$ M. Stoye, ${ }^{107}$ Y. Takahashi, ${ }^{107}$ D. Treille, ${ }^{107}$ A. Triossi, ${ }^{107}$ A. Tsirou, ${ }^{107}$ V. Veckalns, ${ }^{107, \text { uu }}$ G. I. Veres, ${ }^{107, u}$ N. Wardle, ${ }^{107}$ H. K. Wöhri, ${ }^{107}$ A. Zagozdzinska, ${ }^{107, j j}$ W. D. Zeuner, ${ }^{107}$ W. Bertl, ${ }^{108}$ K. Deiters, ${ }^{108}$ W. Erdmann, ${ }^{108}$ R. Horisberger, ${ }^{108}$ Q. Ingram, ${ }^{108}$ H. C. Kaestli, ${ }^{108}$ D. Kotlinski, ${ }^{108}$ U. Langenegger, ${ }^{108}$ T. Rohe, ${ }^{108}$ F. Bachmair, ${ }^{109}$ L. Bäni, ${ }^{109}$ L. Bianchini, ${ }^{109}$ B. Casal, ${ }^{109}$ G. Dissertori, ${ }^{109}$ M. Dittmar, ${ }^{109}$ M. Donegà, ${ }^{109}$ P. Eller,${ }^{109}$ C. Grab,${ }^{109}$ C. Heidegger,${ }^{109}$ D. Hits, ${ }^{109}$ J. Hoss, ${ }^{109}$ G. Kasieczka, ${ }^{109}$ P. Lecomte, ${ }^{109, a}$ W. Lustermann, ${ }^{109}$ B. Mangano, ${ }^{109}$ M. Marionneau, ${ }^{109}$ P. Martinez Ruiz del Arbol,${ }^{109}$ M. Masciovecchio, ${ }^{109}$ M. T. Meinhard, ${ }^{109}$ D. Meister, ${ }^{109}$ F. Micheli, ${ }^{109}$ P. Musella, ${ }^{109}$ F. Nessi-Tedaldi, ${ }^{109}$ F. Pandolfi, ${ }^{109}$ J. Pata, ${ }^{109}$ F. Pauss, ${ }^{109}$ G. Perrin, ${ }^{109}$ L. Perrozzi, ${ }^{109}$ M. Quittnat, ${ }^{109}$ M. Rossini, ${ }^{109}$ M. Schönenberger,${ }^{109}$ A. Starodumov, ${ }^{109, v v}$ M. Takahashi, ${ }^{109}$ V. R. Tavolaro, ${ }^{109}$ K. Theofilatos, ${ }^{109}$ R. Wallny, ${ }^{109}$ T. K. Aarrestad, ${ }^{110}$ C. Amsler, ${ }^{110, w w}$ L. Caminada, ${ }^{110}$ M. F. Canelli, ${ }^{110}$ V. Chiochia, ${ }^{110}$ A. De Cosa, ${ }^{110}$ C. Galloni, ${ }^{110}$ A. Hinzmann, ${ }^{110}$ T. Hreus,${ }^{110}$ B. Kilminster, ${ }^{110}$ C. Lange,${ }^{110}$ J. Ngadiuba, ${ }^{110}$ D. Pinna, ${ }^{110}$ G. Rauco, ${ }^{110}$ P. Robmann, ${ }^{110}$ D. Salerno, ${ }^{110}$ Y. Yang,,${ }^{110}$ K. H. Chen, ${ }^{111}$ T. H. Doan, ${ }^{111}$ Sh. Jain, ${ }^{111}$ R. Khurana, ${ }^{111}$ M. Konyushikhin, ${ }^{111}$ C. M. Kuo, ${ }^{11}$ W. Lin, ${ }^{111}$ Y. J. Lu, ${ }^{111}$ A. Pozdnyakov, ${ }^{111}$ S. S. Yu, ${ }^{111}$ Arun Kumar, ${ }^{112}$ P. Chang, ${ }^{112}$ Y. H. Chang, ${ }^{112}$ Y. W. Chang, ${ }^{112}$ Y. Chao, ${ }^{112}$ K. F. Chen, ${ }^{112}$ P. H. Chen, ${ }^{112}$ C. Dietz, ${ }^{112}$ F. Fiori, ${ }^{112}$ W.-S. Hou, ${ }^{112}$ Y. Hsiung, ${ }^{112}$ Y. F. Liu, ${ }^{112}$ R.-S. Lu, ${ }^{112}$ M. Miñano Moya,${ }^{112}$ J. f. Tsai, ${ }^{112}$ Y. M. Tzeng, ${ }^{112}$ B. Asavapibhop, ${ }^{113}$ 
K. Kovitanggoon, ${ }^{113}$ G. Singh, ${ }^{113}$ N. Srimanobhas, ${ }^{113}$ N. Suwonjandee, ${ }^{113}$ A. Adiguzel, ${ }^{114}$ S. Cerci, ${ }^{114 x x}$ S. Damarseckin, ${ }^{114}$ Z. S. Demiroglu, ${ }^{114}$ C. Dozen, ${ }^{114}$ I. Dumanoglu, ${ }^{114}$ S. Girgis, ${ }^{114}$ G. Gokbulut,${ }^{114}$ Y. Guler, ${ }^{114}$ E. Gurpinar,${ }^{114}$ I. Hos,${ }^{114}$ E. E. Kangal, ${ }^{114, y y}$ A. Kayis Topaksu, ${ }^{114}$ G. Onengut, ${ }^{114, z z}$ K. Ozdemir, ${ }^{114, \text { aaa }}$ S. Ozturk, ${ }^{114, b b b}$ B. Tali, ${ }^{114, x x}$ H. Topakli, ${ }^{114, b b b}$ C. Zorbilmez, ${ }^{114}$ B. Bilin, ${ }^{15}$ S. Bilmis, ${ }^{115}$ B. Isildak, ${ }^{115, c c c}$ G. Karapinar, ${ }^{115, \text { ddd }}$ M. Yalvac, ${ }^{115}$ M. Zeyrek, ${ }^{115}$ E. Gülmez, ${ }^{116}$ M. Kaya ${ }^{116, \text { eee }}$ O. Kaya, ${ }^{116, f f}$ E. A. Yetkin, ${ }^{116, \text { ggg }}$ T. Yetkin, ${ }^{16, \text { hhh }}$ A. Cakir, ${ }^{117}$ K. Cankocak, ${ }^{117}$ S. Sen, ${ }^{117, \text { iii }}$ F. I. Vardarll, ${ }^{117}$ B. Grynyov, ${ }^{118}$ L. Levchuk, ${ }^{119}$ P. Sorokin, ${ }^{119}$ R. Aggleton, ${ }^{120}$ F. Ball, ${ }^{120}$ L. Beck, ${ }^{120}$ J. J. Brooke, ${ }^{120}$ D. Burns, ${ }^{120}$ E. Clement, ${ }^{120}$ D. Cussans, ${ }^{120}$ H. Flacher, ${ }^{120}$ J. Goldstein, ${ }^{120}$ M. Grimes, ${ }^{120}$ G. P. Heath, ${ }^{120}$ H. F. Heath, ${ }^{120}$ J. Jacob, ${ }^{120}$ L. Kreczko, ${ }^{120}$ C. Lucas, ${ }^{120}$ Z. Meng, ${ }^{120}$ D. M. Newbold, ${ }^{120, j j j}$ S. Paramesvaran, ${ }^{120}$ A. Poll, ${ }^{120}$ T. Sakuma, ${ }^{120}$ S. Seif El Nasr-storey, ${ }^{120}$ S. Senkin, ${ }^{120}$ D. Smith, ${ }^{120}$ V. J. Smith, ${ }^{120}$ K. W. Bell, ${ }^{121}$ A. Belyaev, ${ }^{121, k k k}$ C. Brew, ${ }^{121}$ R. M. Brown, ${ }^{121}$ L. Calligaris, ${ }^{121}$ D. Cieri, ${ }^{121}$ D. J. A. Cockerill, ${ }^{121}$ J. A. Coughlan, ${ }^{121}$ K. Harder, ${ }^{121}$ S. Harper, ${ }^{121}$ E. Olaiya, ${ }^{121}$ D. Petyt, ${ }^{121}$ C. H. Shepherd-Themistocleous, ${ }^{121}$ A. Thea, ${ }^{121}$ I. R. Tomalin, ${ }^{121}$ T. Williams, ${ }^{121}$ S. D. Worm, ${ }^{121}$ M. Baber, ${ }^{122}$ R. Bainbridge, ${ }^{122}$ O. Buchmuller, ${ }^{122}$ A. Bundock, ${ }^{122}$ D. Burton, ${ }^{122}$ S. Casasso, ${ }^{122}$ M. Citron, ${ }^{122}$ D. Colling, ${ }^{122}$ L. Corpe ${ }^{122}$ P. Dauncey, ${ }^{122}$ G. Davies, ${ }^{122}$ A. De Wit ${ }^{122}$ M. Della Negra, ${ }^{122}$ P. Dunne, ${ }^{122}$ A. Elwood, ${ }^{122}$ D. Futyan, ${ }^{122}$ Y. Haddad, ${ }^{122}$ G. Hall, ${ }^{122}$ G. Iles, ${ }^{122}$ R. Lane, ${ }^{122}$ R. Lucas, ${ }^{122, j j}$ L. Lyons, ${ }^{122}$ A.-M. Magnan, ${ }^{122}$ S. Malik, ${ }^{122}$ L. Mastrolorenzo, ${ }^{122}$ J. Nash, ${ }^{122}$ A. Nikitenko, ${ }^{122, v v}$ J. Pela, ${ }^{122}$ B. Penning, ${ }^{122}$ M. Pesaresi, ${ }^{122}$ D. M. Raymond, ${ }^{122}$ A. Richards ${ }^{122}$ A. Rose,${ }^{122}$ C. Seez, ${ }^{122}$ A. Tapper, ${ }^{122}$ K. Uchida, ${ }^{122}$ M. Vazquez Acosta, ${ }^{122,111}$ T. Virdee, ${ }^{122, n}$ S. C. Zenz, ${ }^{122}$ J. E. Cole, ${ }^{123}$ P. R. Hobson, ${ }^{123}$ A. Khan, ${ }^{123}$ P. Kyberd, ${ }^{123}$ D. Leslie, ${ }^{123}$ I. D. Reid, ${ }^{123}$ P. Symonds, ${ }^{123}$ L. Teodorescu, ${ }^{123}$ M. Turner, ${ }^{123}$ A. Borzou, ${ }^{124}$ K. Call, ${ }^{124}$ J. Dittmann, ${ }^{124}$ K. Hatakeyama, ${ }^{124}$ H. Liu, ${ }^{124}$ N. Pastika, ${ }^{124}$ O. Charaf, ${ }^{125}$ S. I. Cooper, ${ }^{125}$ C. Henderson, ${ }^{125}$ P. Rumerio, ${ }^{125}$ D. Arcaro, ${ }^{126}$ A. Avetisyan, ${ }^{126}$ T. Bose, ${ }^{126}$ D. Gastler,${ }^{126}$ D. Rankin, ${ }^{126}$ C. Richardson, ${ }^{126}$ J. Rohlf, ${ }^{126}$ L. Sulak, ${ }^{126}$ D. Zou, ${ }^{126}$ J. Alimena, ${ }^{127}$ G. Benelli, ${ }^{127}$ E. Berry, ${ }^{127}$ D. Cutts, ${ }^{127}$ A. Ferapontov, ${ }^{127}$ A. Garabedian, ${ }^{127}$ J. Hakala, ${ }^{127}$ U. Heintz, ${ }^{127}$ O. Jesus, ${ }^{127}$ E. Laird, ${ }^{127}$ G. Landsberg, ${ }^{127}$ Z. Mao, ${ }^{127}$ M. Narain, ${ }^{127}$ S. Piperov, ${ }^{127}$ S. Sagir, ${ }^{127}$ R. Syarif, ${ }^{127}$ R. Breedon, ${ }^{128}$ G. Breto, ${ }^{128}$ M. Calderon De La Barca Sanchez, ${ }^{128}$ S. Chauhan, ${ }^{128}$ M. Chertok, ${ }^{128}$ J. Conway, ${ }^{128}$ R. Conway, ${ }^{128}$ P. T. Cox,${ }^{128}$ R. Erbacher, ${ }^{128}$ C. Flores,${ }^{128}$ G. Funk, ${ }^{128}$ M. Gardner, ${ }^{128}$ W. Ko, ${ }^{128}$ R. Lander, ${ }^{128}$ C. Mclean, ${ }^{128}$ M. Mulhearn, ${ }^{128}$ D. Pellett, ${ }^{128}$ J. Pilot,${ }^{128}$ F. Ricci-Tam, ${ }^{128}$ S. Shalhout ${ }^{128}$ J. Smith, ${ }^{128}$ M. Squires, ${ }^{128}$ D. Stolp, ${ }^{128}$ M. Tripathi, ${ }^{128}$ S. Wilbur, ${ }^{128}$ R. Yohay, ${ }^{128}$ R. Cousins, ${ }^{129}$ P. Everaerts, ${ }^{129}$ A. Florent, ${ }^{129}$ J. Hauser, ${ }^{129}$ M. Ignatenko, ${ }^{129}$ D. Saltzberg, ${ }^{129}$ E. Takasugi, ${ }^{129}$ V. Valuev, ${ }^{129}$ M. Weber, ${ }^{129}$ K. Burt, ${ }^{130}$ R. Clare, ${ }^{130}$ J. Ellison, ${ }^{130}$ J. W. Gary, ${ }^{130}$ G. Hanson, ${ }^{130}$ J. Heilman, ${ }^{130}$ P. Jandir, ${ }^{130}$ E. Kennedy,${ }^{130}$ F. Lacroix, ${ }^{130}$ O. R. Long, ${ }^{130}$ M. Malberti, ${ }^{130}$ M. Olmedo Negrete, ${ }^{130}$ M. I. Paneva, ${ }^{130}$ A. Shrinivas, ${ }^{130}$ H. Wei, ${ }^{130}$ S. Wimpenny, ${ }^{130}$ B. R. Yates, ${ }^{130}$ J. G. Branson, ${ }^{131}$ G. B. Cerati, ${ }^{131}$ S. Cittolin, ${ }^{131}$ R. T. D’Agnolo,${ }^{131}$ M. Derdzinski, ${ }^{131}$ R. Gerosa, ${ }^{131}$ A. Holzner, ${ }^{131}$ R. Kelley, ${ }^{131}$ D. Klein, ${ }^{131}$ J. Letts, ${ }^{131}$ I. Macneill, ${ }^{131}$ D. Olivito, ${ }^{131}$ S. Padhi, ${ }^{131}$ M. Pieri, ${ }^{131}$ M. Sani, ${ }^{131}$ V. Sharma, ${ }^{131}$ S. Simon, ${ }^{131}$ M. Tadel ${ }^{131}$ A. Vartak, ${ }^{131}$ S. Wasserbaech, ${ }^{131, \mathrm{mmm}}$ C. Welke, ${ }^{131}$ J. Wood, ${ }^{131}$ F. Würthwein, ${ }^{131}$ A. Yagil, ${ }^{131}$ G. Zevi Della Porta, ${ }^{131}$ J. Bradmiller-Feld, ${ }^{132}$ C. Campagnari, ${ }^{132}$ A. Dishaw, ${ }^{132}$ V. Dutta, ${ }^{132}$ K. Flowers, ${ }^{132}$ M. Franco Sevilla, ${ }^{132}$ P. Geffert, ${ }^{132}$ C. George, ${ }^{132}$ F. Golf, ${ }^{132}$ L. Gouskos, ${ }^{132}$ J. Gran, ${ }^{132}$ J. Incandela, ${ }^{132}$ N. Mccoll, ${ }^{132}$ S. D. Mullin, ${ }^{132}$ J. Richman, ${ }^{132}$ D. Stuart, ${ }^{132}$ I. Suarez, ${ }^{132}$ C. West,${ }^{132}$ J. Yoo, ${ }^{132}$ D. Anderson, ${ }^{133}$ A. Apresyan, ${ }^{133}$ J. Bendavid, ${ }^{133}$ A. Bornheim, ${ }^{133}$ J. Bunn, ${ }^{133}$ Y. Chen, ${ }^{133}$ J. Duarte, ${ }^{133}$ A. Mott, ${ }^{133}$ H. B. Newman, ${ }^{133}$ C. Pena, ${ }^{133}$ M. Spiropulu, ${ }^{133}$ J. R. Vlimant, ${ }^{133}$ S. Xie, ${ }^{133}$ R. Y. Zhu, ${ }^{133}$ M. B. Andrews, ${ }^{134}$ V. Azzolini, ${ }^{134}$ A. Calamba, ${ }^{134}$ B. Carlson, ${ }^{134}$ T. Ferguson, ${ }^{134}$ M. Paulini, ${ }^{134}$ J. Russ, ${ }^{134}$ M. Sun, ${ }^{134}$ H. Vogel, ${ }^{134}$ I. Vorobiev, ${ }^{134}$ J. P. Cumalat, ${ }^{135}$ W. T. Ford, ${ }^{135}$ F. Jensen, ${ }^{135}$ A. Johnson, ${ }^{135}$ M. Krohn, ${ }^{135}$ T. Mulholland, ${ }^{135}$ K. Stenson, ${ }^{135}$ S. R. Wagner, ${ }^{135}$ J. Alexander,${ }^{136}$ A. Chatterjee, ${ }^{136}$ J. Chaves, ${ }^{136}$ J. Chu,${ }^{136}$ S. Dittmer ${ }^{136}$ N. Eggert, ${ }^{136}$ N. Mirman, ${ }^{136}$ G. Nicolas Kaufman, ${ }^{136}$ J. R. Patterson, ${ }^{136}$ A. Rinkevicius, ${ }^{136}$ A. Ryd, ${ }^{136}$ L. Skinnari, ${ }^{136}$ L. Soffi, ${ }^{136}$ W. Sun, ${ }^{136}$ S. M. Tan, ${ }^{136}$ W. D. Teo, ${ }^{136}$ J. Thom, ${ }^{136}$ J. Thompson, ${ }^{136}$ J. Tucker, ${ }^{136}$ Y. Weng, ${ }^{136}$ P. Wittich, ${ }^{136}$ S. Abdullin, ${ }^{137}$ M. Albrow, ${ }^{137}$ G. Apollinari, ${ }^{137}$ S. Banerjee, ${ }^{137}$ L. A. T. Bauerdick, ${ }^{137}$ A. Beretvas, ${ }^{137}$ J. Berryhill, ${ }^{137}$ P. C. Bhat, ${ }^{137}$ G. Bolla, ${ }^{137}$ K. Burkett, ${ }^{137}$ J. N. Butler, ${ }^{137}$ H. W. K. Cheung, ${ }^{137}$ F. Chlebana, ${ }^{137}$ S. Cihangir, ${ }^{137}$ M. Cremonesi ${ }^{137}$ V. D. Elvira ${ }^{137}$ I. Fisk, ${ }^{137}$ J. Freeman, ${ }^{137}$ E. Gottschalk, ${ }^{137}$ L. Gray,${ }^{137}$ D. Green, ${ }^{137}$ S. Grünendahl, ${ }^{137}$ O. Gutsche ${ }^{137}$ D. Hare ${ }^{137}$ R. M. Harris, ${ }^{137}$ S. Hasegawa, ${ }^{137}$ J. Hirschauer, ${ }^{137}$ Z. Hu, ${ }^{137}$ B. Jayatilaka, ${ }^{137}$ S. Jindariani ${ }^{137}$ M. Johnson, ${ }^{137}$ U. Joshi,${ }^{137}$ B. Klima, ${ }^{137}$ B. Kreis, ${ }^{137}$ S. Lammel,${ }^{137}$ J. Lewis, ${ }^{137}$ J. Linacre,${ }^{137}$ D. Lincoln, ${ }^{137}$ R. Lipton, ${ }^{137}$ T. Liu, ${ }^{137}$ R. Lopes De Sá, ${ }^{137}$ J. Lykken, ${ }^{137}$ K. Maeshima, ${ }^{137}$ J. M. Marraffino, ${ }^{137}$ S. Maruyama, ${ }^{137}$

D. Mason, ${ }^{137}$ P. McBride, ${ }^{137}$ P. Merkel, ${ }^{137}$ S. Mrenna, ${ }^{137}$ S. Nahn, ${ }^{137}$ C. Newman-Holmes, ${ }^{137, a}$ V. O’Dell, ${ }^{137}$ K. Pedro, ${ }^{137}$ O. Prokofyev, ${ }^{137}$ G. Rakness, ${ }^{137}$ E. Sexton-Kennedy, ${ }^{137}$ A. Soha, ${ }^{137}$ W. J. Spalding, ${ }^{137}$ L. Spiegel, ${ }^{137}$ S. Stoynev, ${ }^{137}$ N. Strobbe, ${ }^{137}$ L. Taylor, ${ }^{137}$ S. Tkaczyk, ${ }^{137}$ N. V. Tran, ${ }^{137}$ L. Uplegger,${ }^{137}$ E. W. Vaandering, ${ }^{137}$ C. Vernieri, ${ }^{137}$ 
M. Verzocchi, ${ }^{137}$ R. Vidal, ${ }^{137}$ M. Wang, ${ }^{137}$ H. A. Weber,${ }^{137}$ A. Whitbeck, ${ }^{137}$ D. Acosta, ${ }^{138}$ P. Avery, ${ }^{138}$ P. Bortignon, ${ }^{138}$ D. Bourilkov, ${ }^{138}$ A. Brinkerhoff, ${ }^{138}$ A. Carnes, ${ }^{138}$ M. Carver, ${ }^{138}$ D. Curry, ${ }^{138}$ S. Das, ${ }^{138}$ R. D. Field, ${ }^{138}$ I. K. Furic, ${ }^{138}$ J. Konigsberg, ${ }^{138}$ A. Korytov, ${ }^{138}$ K. Kotov, ${ }^{138}$ P. Ma, ${ }^{138}$ K. Matchev, ${ }^{138}$ H. Mei, ${ }^{138}$ P. Milenovic, ${ }^{138, n n n}$ G. Mitselmakher, ${ }^{138}$ D. Rank, ${ }^{138}$ R. Rossin, ${ }^{138}$ L. Shchutska, ${ }^{138}$ D. Sperka, ${ }^{138}$ N. Terentyev, ${ }^{138}$ L. Thomas, ${ }^{138}$ J. Wang, ${ }^{138}$ S. Wang, ${ }^{138}$ J. Yelton, ${ }^{138}$ S. Linn, ${ }^{139}$ P. Markowitz, ${ }^{139}$ G. Martinez, ${ }^{139}$ J. L. Rodriguez, ${ }^{139}$ A. Ackert, ${ }^{140}$ J. R. Adams, ${ }^{140}$ T. Adams, ${ }^{140}$ A. Askew, ${ }^{140}$ S. Bein, ${ }^{140}$ J. Bochenek, ${ }^{140}$ B. Diamond, ${ }^{140}$ J. Haas, ${ }^{140}$ S. Hagopian, ${ }^{140}$ V. Hagopian, ${ }^{140}$ K. F. Johnson, ${ }^{140}$ A. Khatiwada, ${ }^{140}$ H. Prosper ${ }^{140}$ A. Santra, ${ }^{140}$ M. Weinberg, ${ }^{140}$ M. M. Baarmand, ${ }^{141}$ V. Bhopatkar, ${ }^{141}$ S. Colafranceschi, ${ }^{141,00 o}$ M. Hohlmann, ${ }^{141}$ H. Kalakhety, ${ }^{141}$ D. Noonan, ${ }^{141}$ T. Roy,${ }^{141}$ F. Yumiceva,${ }^{141}$ M. R. Adams, ${ }^{142}$ L. Apanasevich, ${ }^{142}$ D. Berry, ${ }^{142}$ R. R. Betts, ${ }^{142}$ I. Bucinskaite, ${ }^{142}$ R. Cavanaugh, ${ }^{142}$ O. Evdokimov, ${ }^{142}$ L. Gauthier, ${ }^{142}$ C. E. Gerber, ${ }^{142}$ D. J. Hofman, ${ }^{142}$ P. Kurt, ${ }^{142}$ C. O’Brien, ${ }^{142}$ I. D. Sandoval Gonzalez, ${ }^{142}$ P. Turner, ${ }^{142}$ N. Varelas, ${ }^{142}$ Z. Wu, ${ }^{142}$ M. Zakaria, ${ }^{142}$ J. Zhang, ${ }^{142}$ B. Bilki, ${ }^{143, p p p}$ W. Clarida, ${ }^{143}$ K. Dilsiz, ${ }^{143}$ S. Durgut, ${ }^{143}$ R. P. Gandrajula, ${ }^{143}$ M. Haytmyradov, ${ }^{143}$

V. Khristenko, ${ }^{143}$ J.-P. Merlo, ${ }^{143}$ H. Mermerkaya, ${ }^{143, q q 9}$ A. Mestvirishvili, ${ }^{143}$ A. Moeller, ${ }^{143}$ J. Nachtman, ${ }^{143}$ H. Ogul, ${ }^{143}$ Y. Onel, ${ }^{143}$ F. Ozok, ${ }^{143, \text { rrr }}$ A. Penzo, ${ }^{143}$ C. Snyder, ${ }^{143}$ E. Tiras, ${ }^{143}$ J. Wetzel,,${ }^{143}$ K. Yi, ${ }^{143}$ I. Anderson, ${ }^{144}$ B. Blumenfeld, ${ }^{144}$ A. Cocoros, ${ }^{144}$ N. Eminizer, ${ }^{144}$ D. Fehling, ${ }^{144}$ L. Feng, ${ }^{144}$ A. V. Gritsan, ${ }^{144}$ P. Maksimovic, ${ }^{144}$ M. Osherson, ${ }^{144}$ J. Roskes, ${ }^{144}$ U. Sarica, ${ }^{144}$ M. Swartz, ${ }^{144}$ M. Xiao, ${ }^{144}$ Y. Xin, ${ }^{144}$ C. You, ${ }^{144}$ P. Baringer,${ }^{145}$ A. Bean, ${ }^{145}$ C. Bruner, ${ }^{145}$ J. Castle, ${ }^{145}$ R. P. Kenny III, ${ }^{145}$ A. Kropivnitskaya, ${ }^{145}$ D. Majumder, ${ }^{145}$ M. Malek, ${ }^{145}$ W. Mcbrayer, ${ }^{145}$ M. Murray, ${ }^{145}$ S. Sanders, ${ }^{145}$ R. Stringer ${ }^{145}$ Q. Wang, ${ }^{145}$ A. Ivanov, ${ }^{146}$ K. Kaadze, ${ }^{146}$ S. Khalil, ${ }^{146}$ M. Makouski, ${ }^{146}$ Y. Maravin, ${ }^{146}$ A. Mohammadi, ${ }^{146}$ L. K. Saini ${ }^{146}$ N. Skhirtladze, ${ }^{146}$ S. Toda,${ }^{146}$ D. Lange, ${ }^{147}$ F. Rebassoo, ${ }^{147}$ D. Wright,${ }^{147}$ C. Anelli, ${ }^{148}$ A. Baden, ${ }^{148}$ O. Baron, ${ }^{148}$ A. Belloni, ${ }^{148}$ B. Calvert, ${ }^{148}$ S. C. Eno, ${ }^{148}$ C. Ferraioli, ${ }^{148}$ J. A. Gomez,${ }^{148}$ N. J. Hadley, ${ }^{148}$ S. Jabeen, ${ }^{148}$ R. G. Kellogg, ${ }^{148}$ T. Kolberg, ${ }^{148}$ J. Kunkle, ${ }^{148}$ Y. Lu, ${ }^{148}$ A. C. Mignerey, ${ }^{148}$ Y. H. Shin, ${ }^{148}$ A. Skuja, ${ }^{148}$ M. B. Tonjes, ${ }^{148}$ S. C. Tonwar, ${ }^{148}$ A. Apyan, ${ }^{149}$ R. Barbieri, ${ }^{149}$ A. Baty,${ }^{149}$ R. Bi, ${ }^{149}$ K. Bierwagen, ${ }^{149}$ S. Brandt, ${ }^{149}$ W. Busza, ${ }^{149}$ I. A. Cali, ${ }^{149}$ Z. Demiragli, ${ }^{149}$ L. Di Matteo, ${ }^{149}$ G. Gomez Ceballos, ${ }^{149}$ M. Goncharov, ${ }^{149}$ D. Gulhan, ${ }^{149}$ D. Hsu, ${ }^{149}$ Y. Iiyama, ${ }^{149}$ G. M. Innocenti, ${ }^{149}$ M. Klute, ${ }^{149}$ D. Kovalskyi, ${ }^{149}$ K. Krajczar, ${ }^{149}$ Y. S. Lai, ${ }^{149}$ Y.-J. Lee, ${ }^{149}$ A. Levin, ${ }^{149}$ P. D. Luckey, ${ }^{149}$ A. C. Marini, ${ }^{149}$ C. Mcginn, ${ }^{149}$ C. Mironov, ${ }^{149}$ S. Narayanan, ${ }^{149}$ X. Niu, ${ }^{149}$ C. Paus, ${ }^{149}$ C. Roland, ${ }^{149}$ G. Roland, ${ }^{149}$ J. Salfeld-Nebgen, ${ }^{149}$ G. S. F. Stephans, ${ }^{149}$ K. Sumorok, ${ }^{149}$ K. Tatar, ${ }^{149}$ M. Varma, ${ }^{149}$ D. Velicanu, ${ }^{149}$ J. Veverka, ${ }^{149}$ J. Wang, ${ }^{149}$ T. W. Wang, ${ }^{149}$ B. Wyslouch, ${ }^{149}$ M. Yang, ${ }^{149}$ V. Zhukova, ${ }^{149}$ A. C. Benvenuti, ${ }^{150}$ B. Dahmes, ${ }^{150}$ A. Evans, ${ }^{150}$ A. Finkel, ${ }^{150}$ A. Gude ${ }^{150}$ P. Hansen, ${ }^{150}$ S. Kalafut, ${ }^{150}$ S. C. Kao, ${ }^{150}$ K. Klapoetke, ${ }^{150}$ Y. Kubota, ${ }^{150}$ Z. Lesko, ${ }^{150}$ J. Mans, ${ }^{150}$ S. Nourbakhsh, ${ }^{150}$ N. Ruckstuhl, ${ }^{150}$ R. Rusack,${ }^{150}$ N. Tambe,${ }^{150}$ J. Turkewitz, ${ }^{150}$ J. G. Acosta, ${ }^{151}$ S. Oliveros,${ }^{151}$

E. Avdeeva, ${ }^{152}$ R. Bartek,${ }^{152}$ K. Bloom, ${ }^{152}$ S. Bose,${ }^{152}$ D. R. Claes, ${ }^{152}$ A. Dominguez, ${ }^{152}$ C. Fangmeier,,${ }^{152}$

R. Gonzalez Suarez, ${ }^{152}$ R. Kamalieddin, ${ }^{152}$ D. Knowlton, ${ }^{152}$ I. Kravchenko, ${ }^{152}$ F. Meier, ${ }^{152}$ J. Monroy, ${ }^{152}$ F. Ratnikov, ${ }^{152}$ J. E. Siado, ${ }^{152}$ G. R. Snow, ${ }^{152}$ B. Stieger, ${ }^{152}$ M. Alyari, ${ }^{153}$ J. Dolen, ${ }^{153}$ J. George,${ }^{153}$ A. Godshalk, ${ }^{153}$ C. Harrington, ${ }^{153}$ I. Iashvili, ${ }^{153}$ J. Kaisen, ${ }^{153}$ A. Kharchilava, ${ }^{153}$ A. Kumar, ${ }^{153}$ A. Parker, ${ }^{153}$ S. Rappoccio, ${ }^{153}$ B. Roozbahani, ${ }^{153}$ G. Alverson, ${ }^{154}$ E. Barberis, ${ }^{154}$ D. Baumgartel, ${ }^{154}$ M. Chasco, ${ }^{154}$ A. Hortiangtham, ${ }^{154}$ A. Massironi, ${ }^{154}$ D. M. Morse, ${ }^{154}$ D. Nash, ${ }^{154}$ T. Orimoto, ${ }^{154}$ R. Teixeira De Lima, ${ }^{154}$ D. Trocino, ${ }^{154}$ R.-J. Wang, ${ }^{154}$ D. Wood,${ }^{154}$ J. Zhang, ${ }^{154}$ S. Bhattacharya, ${ }^{155}$ K. A. Hahn, ${ }^{155}$ A. Kubik, ${ }^{155}$ J. F. Low, ${ }^{155}$ N. Mucia ${ }^{155}$ N. Odell, ${ }^{155}$ B. Pollack, ${ }^{155}$ M. H. Schmitt,,${ }^{155}$ K. Sung, ${ }^{155}$ M. Trovato, ${ }^{155}$ M. Velasco, ${ }^{155}$ N. Dev, ${ }^{156}$ M. Hildreth, ${ }^{156}$ C. Jessop, ${ }^{156}$ D. J. Karmgard, ${ }^{156}$ N. Kellams, ${ }^{156}$ K. Lannon, ${ }^{156}$ N. Marinelli, ${ }^{156}$ F. Meng, ${ }^{156}$ C. Mueller, ${ }^{156}$ Y. Musienko, ${ }^{156, k \mathrm{k}}$ M. Planer, ${ }^{156}$ A. Reinsvold, ${ }^{156}$ R. Ruchti, ${ }^{156}$ N. Rupprecht, ${ }^{156}$ G. Smith, ${ }^{156}$ S. Taroni, ${ }^{156}$ N. Valls, ${ }^{156}$ M. Wayne, ${ }^{156}$ M. Wolf, ${ }^{156}$ A. Woodard, ${ }^{156}$ L. Antonelli, ${ }^{157}$ J. Brinson, ${ }^{157}$ B. Bylsma, ${ }^{157}$

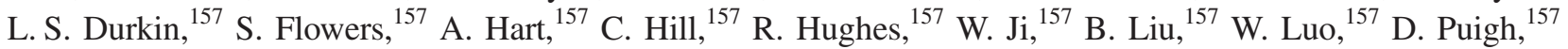
M. Rodenburg, ${ }^{157}$ B. L. Winer, ${ }^{157}$ H. W. Wulsin, ${ }^{157}$ O. Driga, ${ }^{158}$ P. Elmer, ${ }^{158}$ J. Hardenbrook, ${ }^{158}$ P. Hebda, ${ }^{158}$ S. A. Koay, ${ }^{158}$ P. Lujan, ${ }^{158}$ D. Marlow, ${ }^{158}$ T. Medvedeva, ${ }^{158}$ M. Mooney, ${ }^{158}$ J. Olsen, ${ }^{158}$ C. Palmer, ${ }^{158}$ P. Piroué, ${ }^{158}$ D. Stickland, ${ }^{158}$

C. Tully, ${ }^{158}$ A. Zuranski, ${ }^{158}$ S. Malik, ${ }^{159}$ A. Barker, ${ }^{160}$ V. E. Barnes, ${ }^{160}$ D. Benedetti, ${ }^{160}$ L. Gutay, ${ }^{160}$ M. K. Jha, ${ }^{160}$ M. Jones, ${ }^{160}$ A. W. Jung, ${ }^{160}$ K. Jung, ${ }^{160}$ D. H. Miller, ${ }^{160}$ N. Neumeister, ${ }^{160}$ B. C. Radburn-Smith, ${ }^{160}$ X. Shi,${ }^{160}$ J. Sun, ${ }^{160}$ A. Svyatkovskiy, ${ }^{160}$ F. Wang, ${ }^{160}$ W. Xie, ${ }^{160}$ L. Xu, ${ }^{160}$ N. Parashar, ${ }^{161}$ J. Stupak, ${ }^{161}$ A. Adair, ${ }^{162}$ B. Akgun, ${ }^{162}$ Z. Chen, ${ }^{162}$ K. M. Ecklund, ${ }^{162}$ F. J. M. Geurts, ${ }^{162}$ M. Guilbaud, ${ }^{162}$ W. Li,${ }^{162}$ B. Michlin, ${ }^{162}$ M. Northup, ${ }^{162}$ B. P. Padley, ${ }^{162}$ R. Redjimi, ${ }^{162}$ J. Roberts, ${ }^{162}$ J. Rorie, ${ }^{162}$ Z. Tu, ${ }^{162}$ J. Zabel, ${ }^{162}$ B. Betchart, ${ }^{163}$ A. Bodek, ${ }^{163}$ P. de Barbaro, ${ }^{163}$ R. Demina, ${ }^{163}$ Y. t. Duh, ${ }^{163}$ Y. Eshaq, ${ }^{163}$ T. Ferbel, ${ }^{163}$ M. Galanti, ${ }^{163}$ A. Garcia-Bellido, ${ }^{163}$ J. Han, ${ }^{163}$ O. Hindrichs, ${ }^{163}$ A. Khukhunaishvili, ${ }^{163}$ K. H. Lo, ${ }^{163}$ P. Tan, ${ }^{163}$ M. Verzetti, ${ }^{163}$ J. P. Chou, ${ }^{164}$ E. Contreras-Campana, ${ }^{164}$ Y. Gershtein, ${ }^{164}$ T. A. Gómez Espinosa, ${ }^{164}$ E. Halkiadakis, ${ }^{164}$ M. Heindl, ${ }^{164}$ D. Hidas, ${ }^{164}$ E. Hughes, ${ }^{164}$ S. Kaplan, ${ }^{164}$ R. Kunnawalkam Elayavalli, ${ }^{164}$ S. Kyriacou, ${ }^{164}$ 
A. Lath, ${ }^{164}$ K. Nash, ${ }^{164}$ H. Saka, ${ }^{164}$ S. Salur, ${ }^{164}$ S. Schnetzer, ${ }^{164}$ D. Sheffield, ${ }^{164}$ S. Somalwar, ${ }^{164}$ R. Stone,${ }^{164}$ S. Thomas, ${ }^{164}$ P. Thomassen, ${ }^{164}$ M. Walker, ${ }^{164}$ M. Foerster, ${ }^{165}$ J. Heideman, ${ }^{165}$ G. Riley, ${ }^{165}$ K. Rose,${ }^{165}$ S. Spanier, ${ }^{165}$ K. Thapa ${ }^{165}$ O. Bouhali, ${ }^{166, s s s}$ A. Castaneda Hernandez, ${ }^{166, s s s}$ A. Celik, ${ }^{166}$ M. Dalchenko, ${ }^{166}$ M. De Mattia, ${ }^{166}$ A. Delgado, ${ }^{166}$ S. Dildick, ${ }^{166}$ R. Eusebi, ${ }^{166}$ J. Gilmore, ${ }^{166}$ T. Huang, ${ }^{166}$ T. Kamon, ${ }^{166, t t t}$ V. Krutelyov, ${ }^{166}$ R. Mueller, ${ }^{166}$ I. Osipenkov, ${ }^{166}$ Y. Pakhotin, ${ }^{166}$ R. Patel, ${ }^{166}$ A. Perloff, ${ }^{166}$ L. Perniè, ${ }^{166}$ D. Rathjens, ${ }^{166}$ A. Rose, ${ }^{166}$ A. Safonov, ${ }^{166}$ A. Tatarinov, ${ }^{166}$ K. A. Ulmer, ${ }^{166}$ N. Akchurin, ${ }^{167}$ C. Cowden,${ }^{167}$ J. Damgov,${ }^{167}$ C. Dragoiu, ${ }^{167}$ P. R. Dudero, ${ }^{167}$ J. Faulkner, ${ }^{167}$ S. Kunori, ${ }^{167}$ K. Lamichhane, ${ }^{167}$ S. W. Lee, ${ }^{167}$ T. Libeiro, ${ }^{167}$ S. Undleeb, ${ }^{167}$ I. Volobouev, ${ }^{167}$ Z. Wang, ${ }^{167}$ E. Appelt, ${ }^{168}$ A. G. Delannoy, ${ }^{168}$ S. Greene, ${ }^{168}$ A. Gurrola, ${ }^{168}$ R. Janjam, ${ }^{168}$ W. Johns, ${ }^{168}$ C. Maguire, ${ }^{168}$ Y. Mao, ${ }^{168}$ A. Melo, ${ }^{168}$ H. Ni, ${ }^{168}$ P. Sheldon, ${ }^{168}$ S. Tuo, ${ }^{168}$ J. Velkovska, ${ }^{168}$ Q. Xu, ${ }^{168}$ M. W. Arenton, ${ }^{169}$ P. Barria, ${ }^{169}$ B. Cox,${ }^{169}$ B. Francis, ${ }^{169}$ J. Goodell, ${ }^{169}$ R. Hirosky, ${ }^{169}$ A. Ledovskoy, ${ }^{169}$ H. Li ${ }^{169}$ C. Neu, ${ }^{169}$ T. Sinthuprasith, ${ }^{169}$ X. Sun, ${ }^{169}$ Y. Wang, ${ }^{169}$ E. Wolfe, ${ }^{169}$ F. Xia, ${ }^{169}$ C. Clarke, ${ }^{170}$ R. Harr, ${ }^{170}$ P. E. Karchin, ${ }^{170}$ C. Kottachchi Kankanamge Don, ${ }^{170}$ P. Lamichhane, ${ }^{170}$ J. Sturdy, ${ }^{170}$ D. A. Belknap, ${ }^{171}$ D. Carlsmith, ${ }^{171}$ S. Dasu, ${ }^{171}$ L. Dodd, ${ }^{171}$ S. Duric, ${ }^{171}$ B. Gomber, ${ }^{171}$ M. Grothe, ${ }^{171}$ M. Herndon, ${ }^{171}$ A. Hervé, ${ }^{171}$ P. Klabbers, ${ }^{171}$ A. Lanaro, ${ }^{171}$ A. Levine, ${ }^{171}$ K. Long, ${ }^{171}$ R. Loveless, ${ }^{171}$ A. Mohapatra, ${ }^{171}$ I. Ojalvo, ${ }^{171}$ T. Perry, ${ }^{171}$ G. A. Pierro, ${ }^{171}$ G. Polese, ${ }^{171}$ T. Ruggles, ${ }^{171}$ T. Sarangi, ${ }^{171}$ A. Savin, ${ }^{171}$ A. Sharma, ${ }^{171}$ N. Smith, ${ }^{171}$ W. H. Smith, ${ }^{171}$ D. Taylor, ${ }^{171}$ P. Verwilligen, ${ }^{171}$ and N. Woods ${ }^{171}$

(CMS Collaboration)

\author{
${ }^{1}$ Yerevan Physics Institute, Yerevan, Armenia \\ ${ }^{2}$ Institut für Hochenergiephysik der OeAW, Wien, Austria \\ ${ }^{3}$ National Centre for Particle and High Energy Physics, Minsk, Belarus \\ ${ }^{4}$ Universiteit Antwerpen, Antwerpen, Belgium \\ ${ }^{5}$ Vrije Universiteit Brussel, Brussel, Belgium \\ ${ }^{6}$ Université Libre de Bruxelles, Bruxelles, Belgium \\ ${ }^{7}$ Ghent University, Ghent, Belgium \\ ${ }^{8}$ Université Catholique de Louvain, Louvain-la-Neuve, Belgium \\ ${ }^{9}$ Université de Mons, Mons, Belgium \\ ${ }^{10}$ Centro Brasileiro de Pesquisas Fisicas, Rio de Janeiro, Brazil \\ ${ }^{11}$ Universidade do Estado do Rio de Janeiro, Rio de Janeiro, Brazil \\ ${ }^{12 a}$ Universidade Estadual Paulista, São Paulo, Brazil \\ ${ }^{12 \mathrm{~b}}$ Universidade Federal do ABC, São Paulo, Brazil \\ ${ }^{13}$ Institute for Nuclear Research and Nuclear Energy, Sofia, Bulgaria \\ ${ }^{14}$ University of Sofia, Sofia, Bulgaria \\ ${ }^{15}$ Beihang University, Beijing, China \\ ${ }^{16}$ Institute of High Energy Physics, Beijing, China \\ ${ }^{17}$ State Key Laboratory of Nuclear Physics and Technology, Peking University, Beijing, China \\ ${ }^{18}$ Universidad de Los Andes, Bogota, Colombia \\ ${ }^{19}$ University of Split, Faculty of Electrical Engineering, Mechanical Engineering and Naval Architecture, \\ Split, Croatia \\ ${ }^{20}$ University of Split, Faculty of Science, Split, Croatia \\ ${ }^{21}$ Institute Rudjer Boskovic, Zagreb, Croatia \\ ${ }^{22}$ University of Cyprus, Nicosia, Cyprus \\ ${ }^{23}$ Charles University, Prague, Czech Republic \\ ${ }^{24}$ Universidad San Francisco de Quito, Quito, Ecuador \\ ${ }^{25}$ Academy of Scientific Research and Technology of the Arab Republic of Egypt, Egyptian Network of \\ High Energy Physics, Cairo, Egypt \\ ${ }^{26}$ National Institute of Chemical Physics and Biophysics, Tallinn, Estonia \\ ${ }^{27}$ Department of Physics, University of Helsinki, Helsinki, Finland \\ ${ }^{28}$ Helsinki Institute of Physics, Helsinki, Finland \\ ${ }^{29}$ Lappeenranta University of Technology, Lappeenranta, Finland \\ ${ }^{30}$ DSM/IRFU, CEA/Saclay, Gif-sur-Yvette, France \\ ${ }^{31}$ Laboratoire Leprince-Ringuet, Ecole Polytechnique, IN2P3-CNRS, Palaiseau, France \\ ${ }^{32}$ Institut Pluridisciplinaire Hubert Curien, Université de Strasbourg, Université de Haute Alsace \\ Mulhouse, CNRS/IN2P3, Strasbourg, France
}


${ }^{33}$ Centre de Calcul de l'Institut National de Physique Nucleaire et de Physique des Particules, CNRS/ IN2P3, Villeurbanne, France

${ }^{34}$ Université de Lyon, Université Claude Bernard Lyon 1, CNRS-IN2P3, Institut de Physique Nucléaire de Lyon, Villeurbanne, France

${ }^{35}$ Georgian Technical University, Tbilisi, Georgia

${ }^{36}$ Tbilisi State University, Tbilisi, Georgia

${ }^{37}$ RWTH Aachen University, I. Physikalisches Institut, Aachen, Germany

${ }^{38}$ RWTH Aachen University, III. Physikalisches Institut A, Aachen, Germany

${ }^{39}$ RWTH Aachen University, III. Physikalisches Institut B, Aachen, Germany

${ }^{40}$ Deutsches Elektronen-Synchrotron, Hamburg, Germany

${ }^{41}$ University of Hamburg, Hamburg, Germany

${ }^{42}$ Institut für Experimentelle Kernphysik, Karlsruhe, Germany

${ }^{43}$ Institute of Nuclear and Particle Physics (INPP), NCSR Demokritos, Aghia Paraskevi, Greece

${ }^{44}$ National and Kapodistrian University of Athens, Athens, Greece

${ }^{45}$ University of Ioánnina, Ioánnina, Greece

${ }^{46}$ MTA-ELTE Lendület CMS Particle and Nuclear Physics Group,

Eötvös Loránd University, Budapest, Hungary

${ }^{47}$ Wigner Research Centre for Physics, Budapest, Hungary

${ }^{48}$ Institute of Nuclear Research ATOMKI, Debrecen, Hungary

${ }^{49}$ University of Debrecen, Debrecen, Hungary

${ }^{50}$ National Institute of Science Education and Research, Bhubaneswar, India

${ }^{51}$ Panjab University, Chandigarh, India

${ }^{52}$ University of Delhi, Delhi, India

${ }^{53}$ Saha Institute of Nuclear Physics, Kolkata, India

${ }^{54}$ Bhabha Atomic Research Centre, Mumbai, India

${ }^{55}$ Tata Institute of Fundamental Research, Mumbai, India

${ }^{56}$ Indian Institute of Science Education and Research (IISER), Pune, India

${ }^{57}$ Institute for Research in Fundamental Sciences (IPM), Tehran, Iran

${ }^{58}$ University College Dublin, Dublin, Ireland

${ }^{59 a}$ INFN Sezione di Bari, Bari, Italy

${ }^{59 \mathrm{~b}}$ Università di Bari, Bari, Italy

${ }^{59 \mathrm{c}}$ Politecnico di Bari, Bari, Italy

${ }^{60 \mathrm{a}}$ INFN Sezione di Bologna, Bologna, Italy

${ }^{60 \mathrm{~b}}$ Università di Bologna, Bologna, Italy

${ }^{61 \mathrm{a} I N F N}$ Sezione di Catania, Catania, Italy

${ }^{61 \mathrm{~b}}$ Università di Catania, Catania, Italy

${ }^{62 \mathrm{a}}$ INFN Sezione di Firenze, Firenze, Italy

${ }^{62 \mathrm{~b}}$ Università di Firenze, Firenze, Italy

${ }^{63}$ INFN Laboratori Nazionali di Frascati, Frascati, Italy

${ }^{64 a}$ INFN Sezione di Genova, Genova, Italy

${ }^{64 \mathrm{~b}}$ Università di Genova, Genova, Italy

${ }^{65 a}$ INFN Sezione di Milano-Bicocca, Milano, Italy

${ }^{65 \mathrm{~b}}$ Università di Milano-Bicocca, Milano, Italy

${ }^{66 a}$ INFN Sezione di Napoli, Napoli, Italy

${ }^{66 \mathrm{~b}}$ Università di Napoli 'Federico II', Napoli, Italy

${ }^{66 c}$ Università della Basilicata, Potenza, Italy

${ }^{66 \mathrm{~d}}$ Università G. Marconi, Roma, Italy

${ }^{67 a}$ INFN Sezione di Padova, Padova, Italy

${ }^{67 \mathrm{~b}}$ Università di Padova, Padova, Italy

${ }^{67 \mathrm{c}}$ Università di Trento, Trento, Italy

${ }^{68 a}$ INFN Sezione di Pavia, Pavia, Italy

${ }^{68 \mathrm{~b}}$ Università di Pavia, Pavia, Italy

${ }^{69}$ INFN Sezione di Perugia, Perugia, Italy

${ }^{69 \mathrm{~b}}$ Università di Perugia, Perugia, Italy

${ }^{70 a}$ INFN Sezione di Pisa, Pisa, Italy

${ }^{70 \mathrm{~b}}$ Università di Pisa, Pisa, Italy

${ }^{70 c}$ Scuola Normale Superiore di Pisa, Pisa, Italy

${ }^{71 \mathrm{a}}$ INFN Sezione di Roma

${ }^{71 \mathrm{~b}}$ Università di Roma

${ }^{72 \mathrm{a}}$ INFN Sezione di Torino, Torino, Italy 


\author{
${ }^{72 \mathrm{~b}}$ Università di Torino, Torino, Italy \\ ${ }^{72 \mathrm{c}}$ Università del Piemonte Orientale, Novara, Italy \\ ${ }^{73 a}$ INFN Sezione di Trieste, Trieste, Italy \\ ${ }^{73 b}$ Università di Trieste, Trieste, Italy \\ ${ }^{74}$ Kangwon National University, Chunchon, Korea \\ ${ }^{75}$ Kyungpook National University, Daegu, Korea \\ ${ }^{76}$ Chonbuk National University, Jeonju, Korea \\ ${ }^{77}$ Chonnam National University, Institute for Universe and Elementary Particles, Kwangju, Korea \\ ${ }^{78}$ Korea University, Seoul, Korea \\ ${ }^{79}$ Seoul National University, Seoul, Korea \\ ${ }^{80}$ University of Seoul, Seoul, Korea \\ ${ }^{81}$ Sungkyunkwan University, Suwon, Korea \\ ${ }^{82}$ Vilnius University, Vilnius, Lithuania \\ ${ }^{83}$ National Centre for Particle Physics, Universiti Malaya, Kuala Lumpur, Malaysia \\ ${ }^{84}$ Centro de Investigacion y de Estudios Avanzados del IPN, Mexico City, Mexico \\ ${ }^{85}$ Universidad Iberoamericana, Mexico City, Mexico \\ ${ }^{86}$ Benemerita Universidad Autonoma de Puebla, Puebla, Mexico \\ ${ }^{87}$ Universidad Autónoma de San Luis Potosí, San Luis Potosí, Mexico \\ ${ }^{88}$ University of Auckland, Auckland, New Zealand \\ ${ }^{89}$ University of Canterbury, Christchurch, New Zealand \\ ${ }^{90}$ National Centre for Physics, Quaid-I-Azam University, Islamabad, Pakistan \\ ${ }^{91}$ National Centre for Nuclear Research, Swierk, Poland \\ ${ }^{92}$ Institute of Experimental Physics, Faculty of Physics, University of Warsaw, Warsaw, Poland \\ ${ }^{93}$ Laboratório de Instrumentação e Física Experimental de Partículas, Lisboa, Portugal \\ ${ }^{94}$ Joint Institute for Nuclear Research, Dubna, Russia \\ ${ }^{95}$ Petersburg Nuclear Physics Institute, Gatchina (St. Petersburg), Russia \\ ${ }^{96}$ Institute for Nuclear Research, Moscow, Russia \\ ${ }^{97}$ Institute for Theoretical and Experimental Physics, Moscow, Russia \\ ${ }^{98}$ National Research Nuclear University 'Moscow Engineering Physics Institute' (MEPhI), \\ Moscow, Russia \\ ${ }^{99}$ P.N. Lebedev Physical Institute, Moscow, Russia \\ ${ }^{100}$ Skobeltsyn Institute of Nuclear Physics, Lomonosov Moscow State University, Moscow, Russia \\ ${ }^{101}$ State Research Center of Russian Federation, Institute for High Energy Physics, Protvino, Russia \\ ${ }^{102}$ University of Belgrade, Faculty of Physics and Vinca Institute of Nuclear Sciences, Belgrade, Serbia \\ ${ }^{103}$ Centro de Investigaciones Energéticas Medioambientales y Tecnológicas (CIEMAT), Madrid, Spain \\ ${ }^{104}$ Universidad Autónoma de Madrid, Madrid, Spain \\ ${ }^{105}$ Universidad de Oviedo, Oviedo, Spain \\ ${ }^{106}$ Instituto de Física de Cantabria (IFCA), CSIC-Universidad de Cantabria, Santander, Spain \\ ${ }^{107}$ CERN, European Organization for Nuclear Research, Geneva, Switzerland \\ ${ }^{108}$ Paul Scherrer Institut, Villigen, Switzerland \\ ${ }^{109}$ Institute for Particle Physics, ETH Zurich, Zurich, Switzerland \\ ${ }^{110}$ Universität Zürich, Zurich, Switzerland \\ ${ }^{111}$ National Central University, Chung-Li, Taiwan \\ ${ }^{112}$ National Taiwan University (NTU), Taipei, Taiwan \\ ${ }^{113}$ Chulalongkorn University, Faculty of Science, Department of Physics, Bangkok, Thailand \\ ${ }^{114}$ Cukurova University, Adana, Turkey \\ ${ }^{115}$ Middle East Technical University, Physics Department, Ankara, Turkey \\ ${ }^{116}$ Bogazici University, Istanbul, Turkey \\ ${ }^{117}$ Istanbul Technical University, Istanbul, Turkey \\ ${ }^{118}$ Institute for Scintillation Materials of National Academy of Science of Ukraine, Kharkov, Ukraine \\ ${ }^{119}$ National Scientific Center, Kharkov Institute of Physics and Technology, Kharkov, Ukraine \\ ${ }^{120}$ University of Bristol, Bristol, United Kingdom \\ ${ }^{121}$ Rutherford Appleton Laboratory, Didcot, United Kingdom \\ ${ }^{122}$ Imperial College, London, United Kingdom \\ ${ }^{123}$ Brunel University, Uxbridge, United Kingdom \\ ${ }^{124}$ Baylor University, Waco, Texas, USA \\ ${ }^{125}$ The University of Alabama, Tuscaloosa, Alabama, USA \\ ${ }^{126}$ Boston University, Boston, Massachusetts, USA \\ ${ }^{127}$ Brown University, Providence, Rhode Island, USA \\ ${ }^{128}$ University of California, Davis, California, USA
}




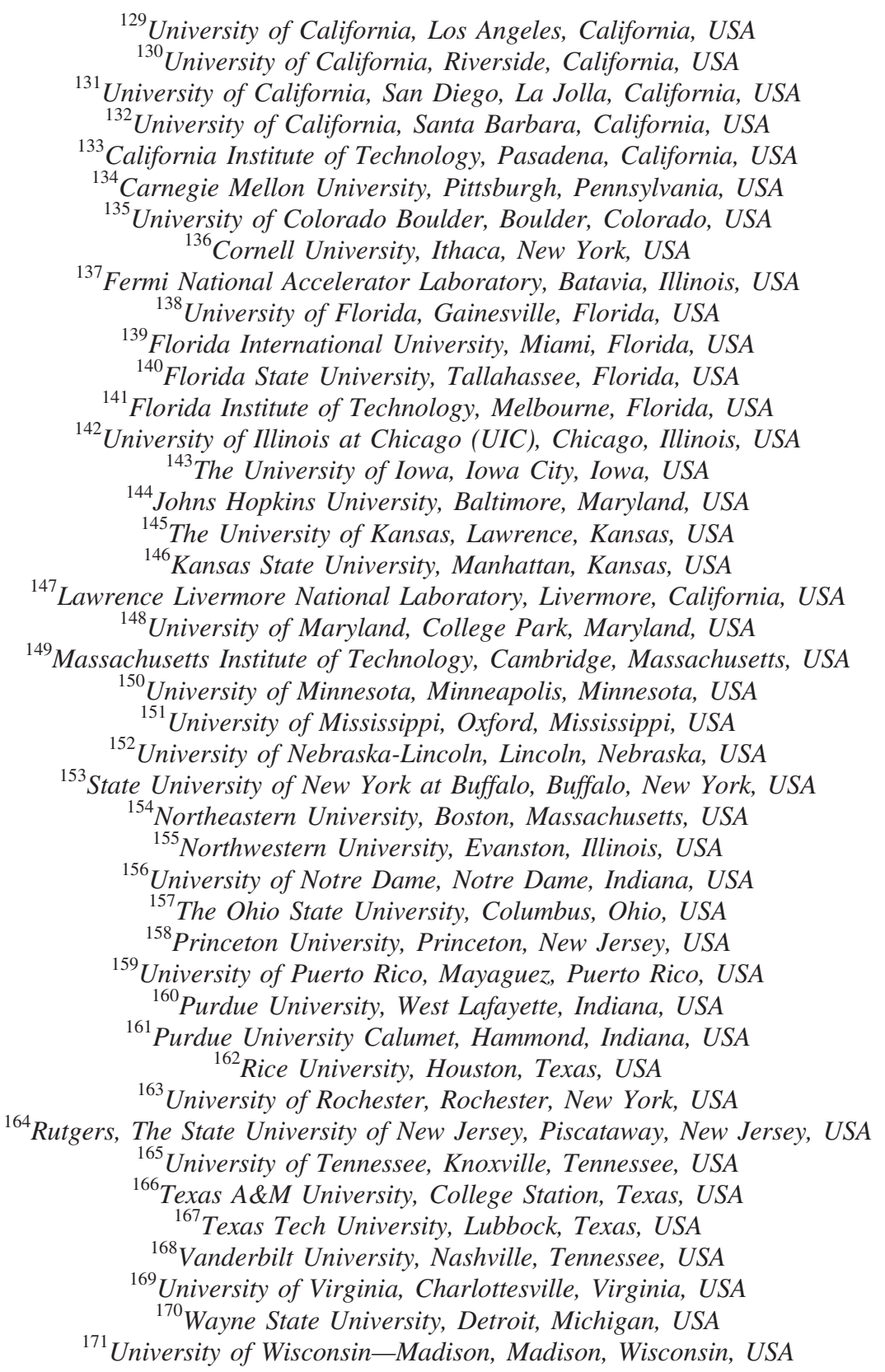

${ }^{\mathrm{a}}$ Deceased.

${ }^{\mathrm{b}}$ Also at Vienna University of Technology, Vienna, Austria.

${ }^{\mathrm{c}}$ Also at State Key Laboratory of Nuclear Physics and Technology, Peking University, Beijing, China.

${ }^{\mathrm{d}}$ Also at Institut Pluridisciplinaire Hubert Curien, Université de Strasbourg, Université de Haute Alsace Mulhouse, CNRS/IN2P3, Strasbourg, France.

${ }^{\mathrm{e}}$ Also at Universidade Estadual de Campinas, Campinas, Brazil.

${ }^{\mathrm{f}}$ Also at Centre National de la Recherche Scientifique (CNRS)—IN2P3, Paris, France.

${ }^{\mathrm{g}}$ Also at Université Libre de Bruxelles, Bruxelles, Belgium.

${ }^{\mathrm{h}}$ Also at Laboratoire Leprince-Ringuet, Ecole Polytechnique, IN2P3-CNRS, Palaiseau, France.

${ }^{\mathrm{i}}$ Also at Joint Institute for Nuclear Research, Dubna, Russia.

${ }^{\mathrm{j}}$ Also at British University in Egypt, Cairo, Egypt.

${ }^{\mathrm{k}}$ Also at Zewail City of Science and Technology, Zewail, Egypt.

${ }^{1}$ Also at Ain Shams University, Cairo, Egypt.

${ }^{\mathrm{m}}$ Also at Université de Haute Alsace, Mulhouse, France.

${ }^{\mathrm{n}}$ Also at CERN, European Organization for Nuclear Research, Geneva, Switzerland.

${ }^{\circ}$ Also at Skobeltsyn Institute of Nuclear Physics, Lomonosov Moscow State University, Moscow, Russia. 
${ }^{\mathrm{p}}$ Also at Tbilisi State University, Tbilisi, Georgia.

${ }^{\mathrm{q}}$ Also at RWTH Aachen University, III. Physikalisches Institut A, Aachen, Germany.

${ }^{\mathrm{r}}$ Also at University of Hamburg, Hamburg, Germany.

${ }^{\mathrm{s}}$ Also at Brandenburg University of Technology, Cottbus, Germany.

${ }^{\mathrm{t}}$ Also at Institute of Nuclear Research ATOMKI, Debrecen, Hungary.

"Also at MTA-ELTE Lendület CMS Particle and Nuclear Physics Group, Eötvös Loránd University.

${ }^{v}$ Also at University of Debrecen, Debrecen, Hungary.

${ }^{w}$ Also at Indian Institute of Science Education and Research, Bhopal, India.

${ }^{\mathrm{x}}$ Also at University of Visva-Bharati, Santiniketan, India.

${ }^{y}$ Also at King Abdulaziz University, Jeddah, Saudi Arabia.

${ }^{\mathrm{z}}$ Also at University of Ruhuna, Matara, Sri Lanka.

${ }^{\text {aa }}$ Also at Isfahan University of Technology, Isfahan, Iran.

${ }^{\mathrm{bb}}$ Also at University of Tehran, Department of Engineering Science, Tehran, Iran.

${ }^{c c}$ Also at Plasma Physics Research Center, Science and Research Branch, Islamic Azad University, Tehran, Iran.

${ }^{\mathrm{dd}}$ Also at Università degli Studi di Siena, Siena, Italy.

${ }^{e e}$ Also at Purdue University, West Lafayette, USA.

${ }^{\mathrm{ff}}$ Also at Hanyang University, Seoul, Korea.

${ }^{\mathrm{gg}}$ Also at International Islamic University of Malaysia, Kuala Lumpur, Malaysia.

${ }^{\text {hh }}$ Also at Malaysian Nuclear Agency, MOSTI, Kajang, Malaysia.

${ }^{i i}$ Also at Consejo Nacional de Ciencia y Tecnología, Mexico city, Mexico.

${ }^{\mathrm{jj}}$ Also at Warsaw University of Technology, Institute of Electronic Systems, Warsaw, Poland.

${ }^{\mathrm{kk}}$ Also at Institute for Nuclear Research, Moscow, Russia.

"Also at National Research Nuclear University 'Moscow Engineering Physics Institute' (MEPhI), Moscow, Russia.

${ }^{\mathrm{mm}}$ Also at St. Petersburg State Polytechnical University, St. Petersburg, Russia.

${ }^{\mathrm{nn}}$ Also at University of Florida, Gainesville, USA.

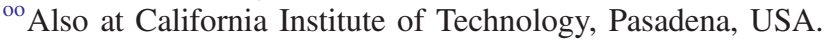

${ }^{\mathrm{pp}}$ Also at Faculty of Physics, University of Belgrade, Belgrade, Serbia.

${ }^{\mathrm{qq}}$ Also at INFN Sezione di Roma, Università di Roma, Roma, Italy.

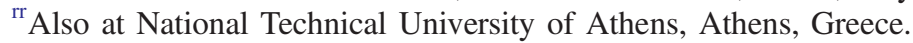

${ }^{\text {ss }}$ Also at Scuola Normale e Sezione dell'INFN, Pisa, Italy.

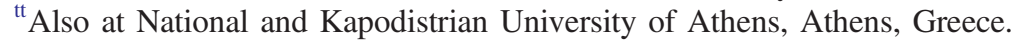

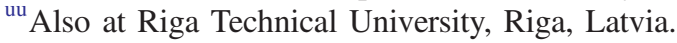

${ }^{\mathrm{vv}}$ Also at Institute for Theoretical and Experimental Physics, Moscow, Russia.

${ }^{\text {ww }}$ Also at Albert Einstein Center for Fundamental Physics, Bern, Switzerland.

${ }^{\mathrm{xx}}$ Also at Adiyaman University, Adiyaman, Turkey.

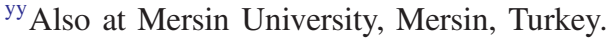

${ }^{\mathrm{zz}}$ Also at Cag University, Mersin, Turkey.

${ }^{\text {aaa } A l s o ~ a t ~ P i r i ~ R e i s ~ U n i v e r s i t y, ~ I s t a n b u l, ~ T u r k e y . ~}$

${ }^{b b b}$ Also at Gaziosmanpasa University, Tokat, Turkey.

${ }^{c c c}$ Also at Ozyegin University, Istanbul, Turkey.

${ }^{\text {ddd }}$ Also at Izmir Institute of Technology, Izmir, Turkey.

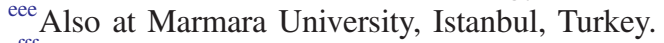

${ }^{\mathrm{fff}}$ Also at Kafkas University, Kars, Turkey.

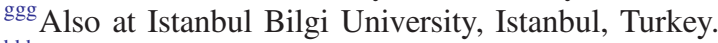

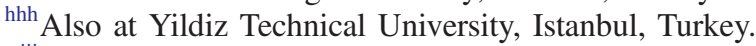

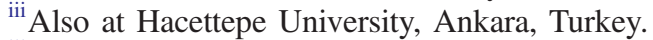

${ }^{j j j}$ Also at Rutherford Appleton Laboratory, Didcot, United Kingdom.

${ }^{k k k}$ Also at School of Physics and Astronomy, University of Southampton, Southampton, United Kingdom.

${ }^{111}$ Also at Instituto de Astrofísica de Canarias, La Laguna, Spain.

${ }^{\mathrm{mmm}}$ Also at Utah Valley University, Orem, USA.

${ }^{n n n}$ Also at University of Belgrade, Faculty of Physics and Vinca Institute of Nuclear Sciences, Belgrade, Serbia.

${ }^{000}$ Also at Facoltà Ingegneria, Università di Roma, Roma, Italy.

${ }^{\mathrm{ppp}}$ Also at Argonne National Laboratory, Argonne, USA.

${ }^{\mathrm{qqq}}$ Also at Erzincan University, Erzincan, Turkey.

${ }^{\mathrm{rrr}}$ Also at Mimar Sinan University, Istanbul, Istanbul, Turkey.

${ }^{\text {sss }}$ Also at Texas A\&M University at Qatar, Doha, Qatar.

${ }^{\text {ttt }}$ Also at Kyungpook National University, Daegu, Korea. 